Supporting Information for

\title{
Chemistry of 2,14-Dithiacalix[4]arene: Alkylation and Conformational Behavior of Peralkylated Products
}

Daniel Kortus, ${ }^{\dagger}$ Jiří Mikšátko, ${ }^{\dagger}$ Ondřej Kundrát, ${ }^{\dagger}$ Martin Babor, ${ }^{\ddagger}$ Václav Eigner, ${ }^{\S}$ Hana Dvořáková ${ }^{\ddagger}$ and Pavel Lhoták ${ }^{+*}$

'Department of Organic Chemistry, University of Chemistry and Technology, Prague (UCTP), Technická 5, 16628 Prague 6, Czech Republic

‡Department of Solid State Chemistry, UCTP, 16628 Prague 6, Czech Republic.

§Institute of Physics AS CR v.v.i., Na Slovance 2, 18221 Prague 8, Czech Republic.

‡Laboratory of NMR spectroscopy, UCTP, 16628 Prague 6, Czech Republic.

*Corresponding author: Ihotakp@vscht.cz 


\section{Table of Contents}

1. General numbering of new synthesized compounds ........................................ 3

2. Copies of spectral characterization of new compounds.....................................S4

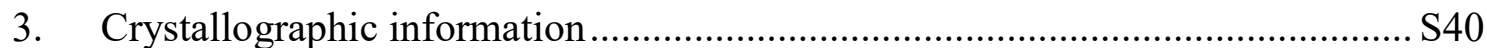

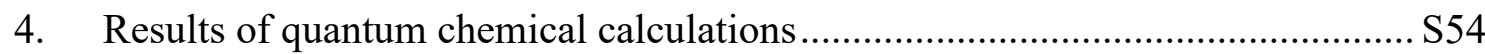


1. GENERAL NUMBERING OF NEW SYNTHESIZED COMPOUNDS

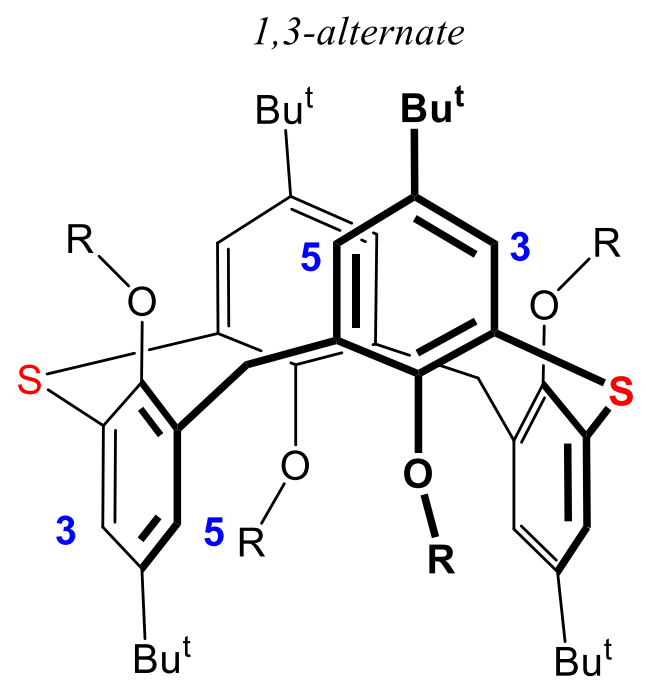

\author{
1,2(S)-alternate
}

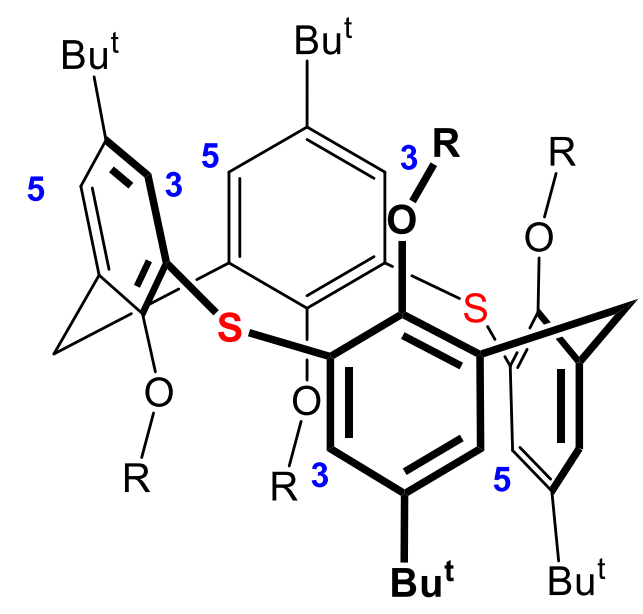

cone

partial cone
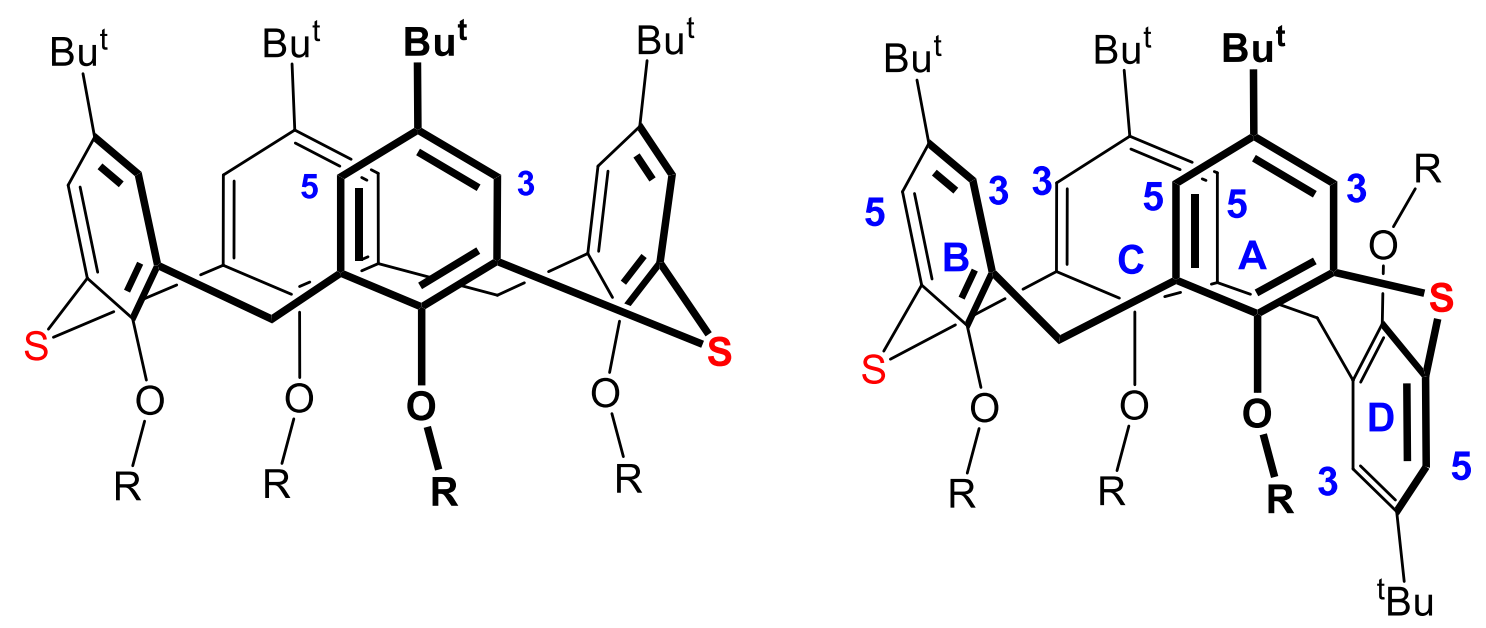

Figure S1: General numbering of new synthetized compounds 


\section{COPIES OF SPECTRAL CHARACTERIZATION OF NEW COMPOUNDS}

\subsection{Compound 2}

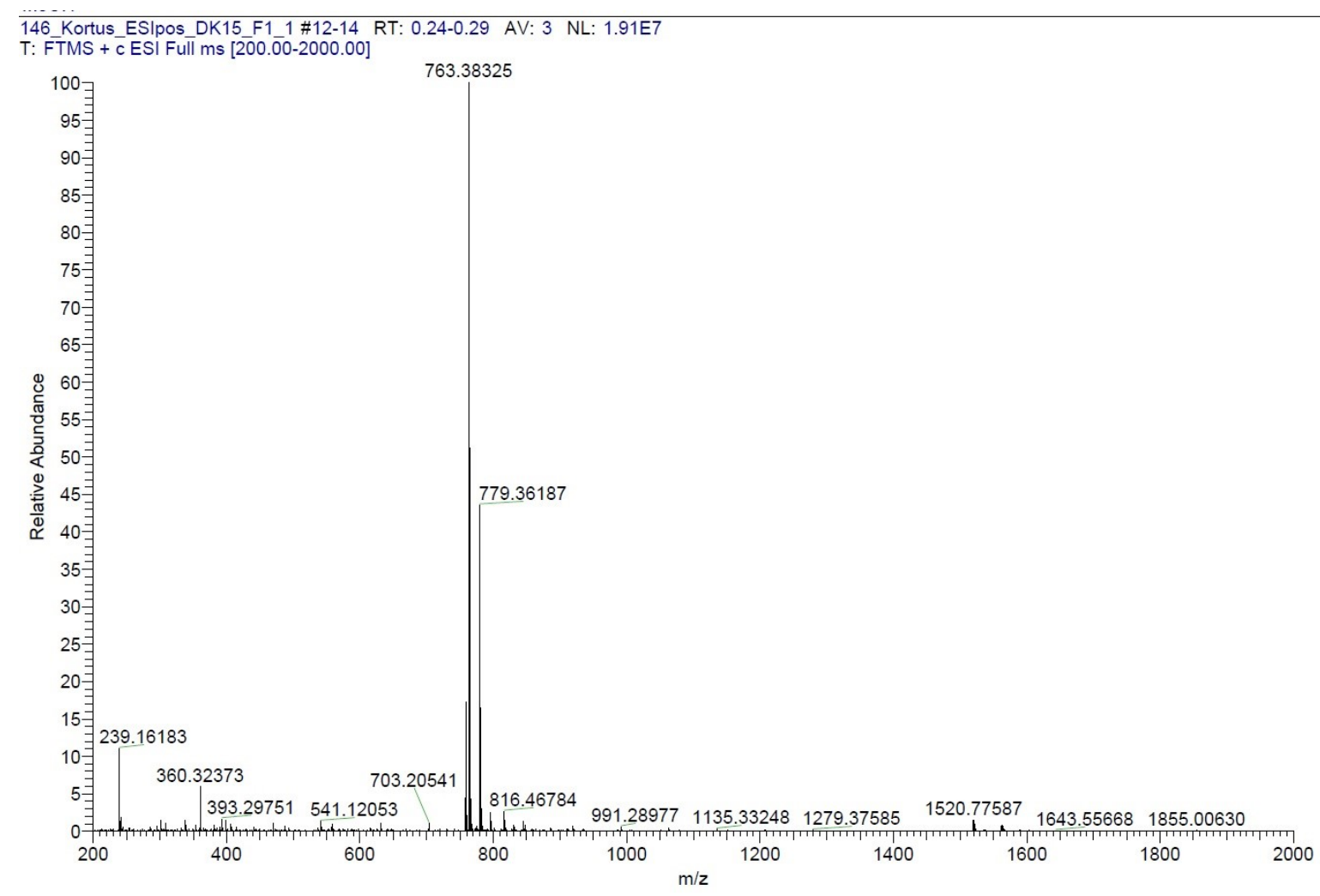

Figure S2: HRMS of compound $\left.2(E S)^{+}\right)$

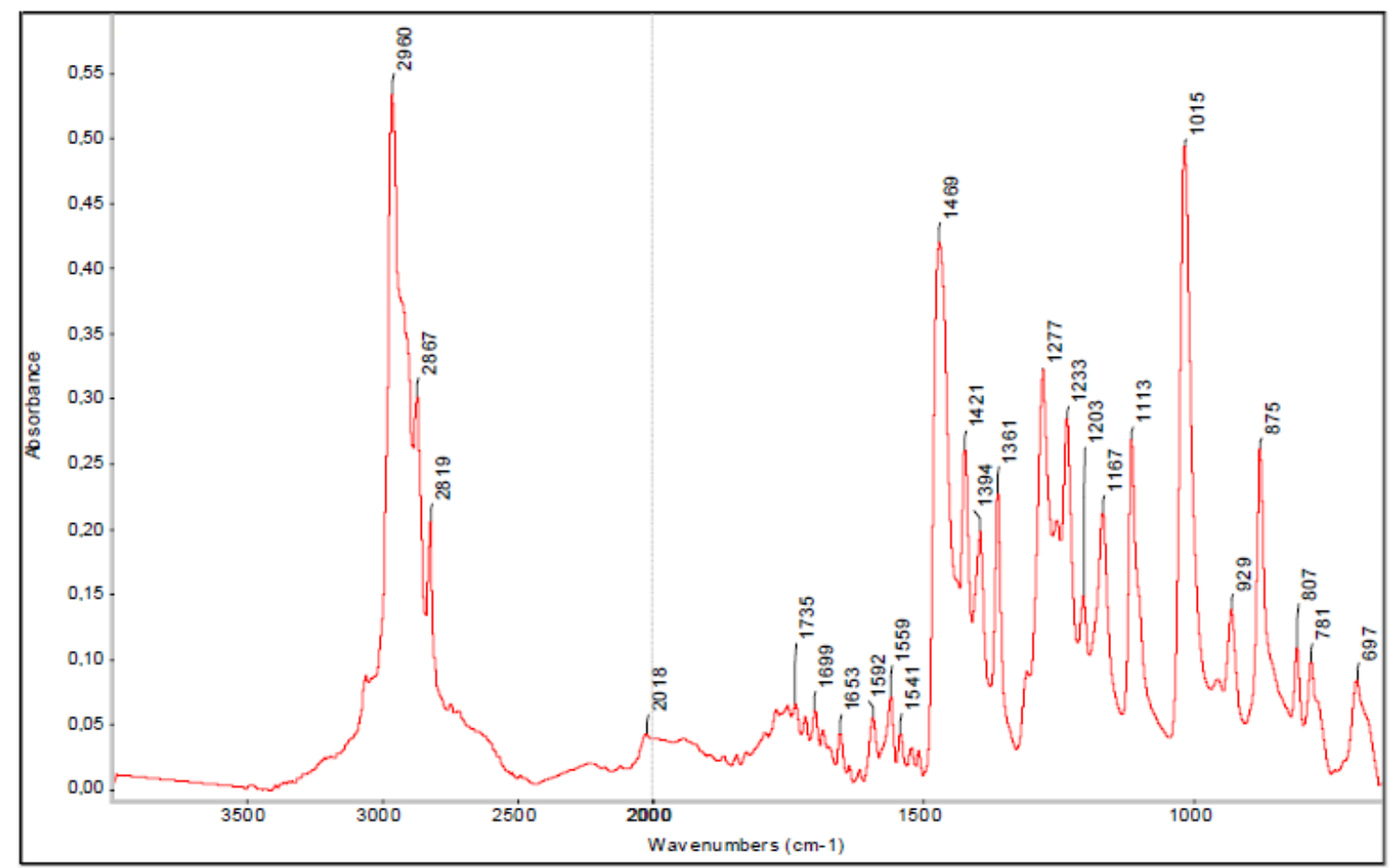

Figure S3: IR of compound $2(\mathrm{KBr})$ 


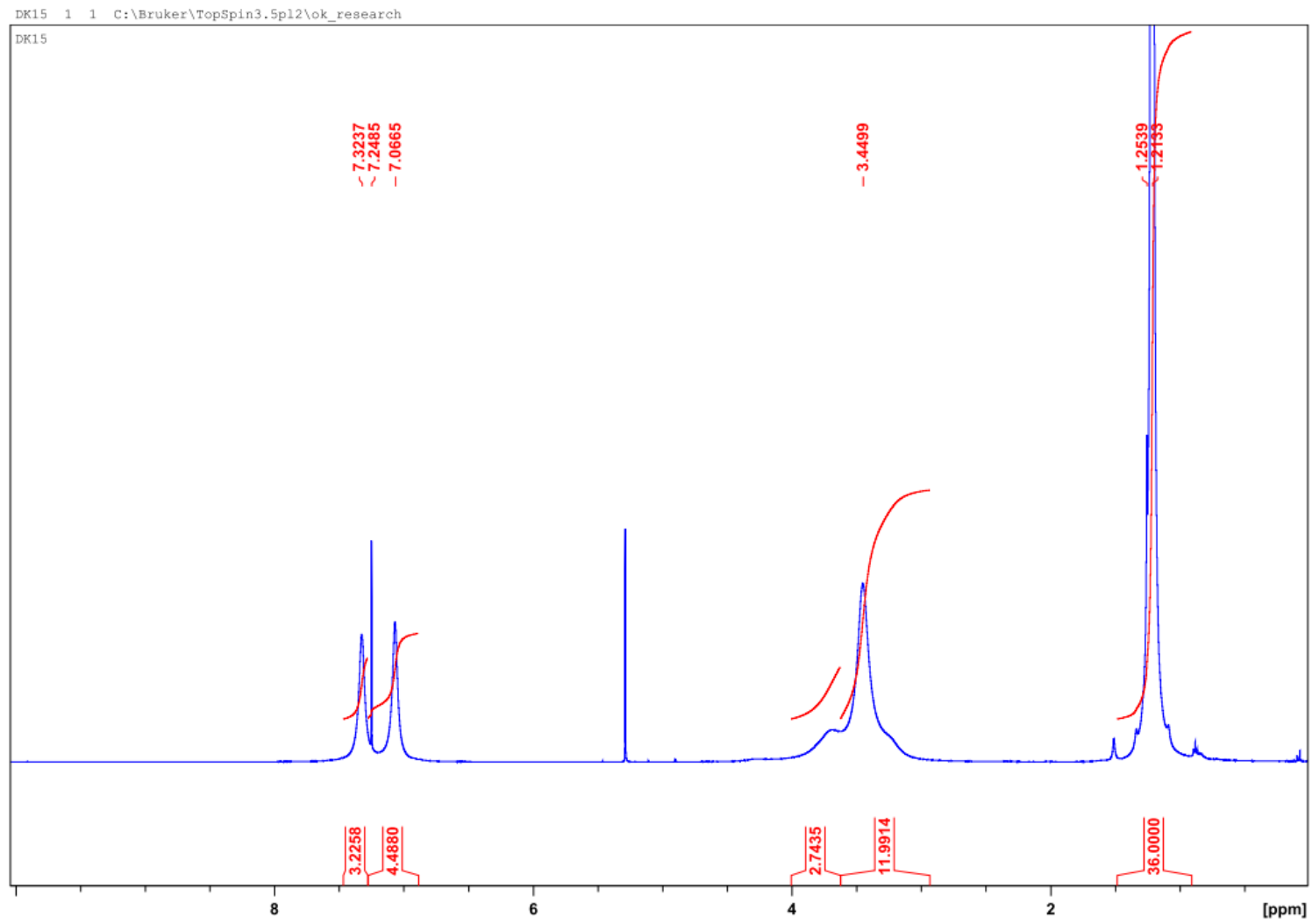

Figure S4: ${ }^{1} \mathrm{H} \mathrm{NMR}$ of compound $2\left(\mathrm{CDCl}_{3}, 300 \mathrm{MHz}, 298 \mathrm{~K}\right)$

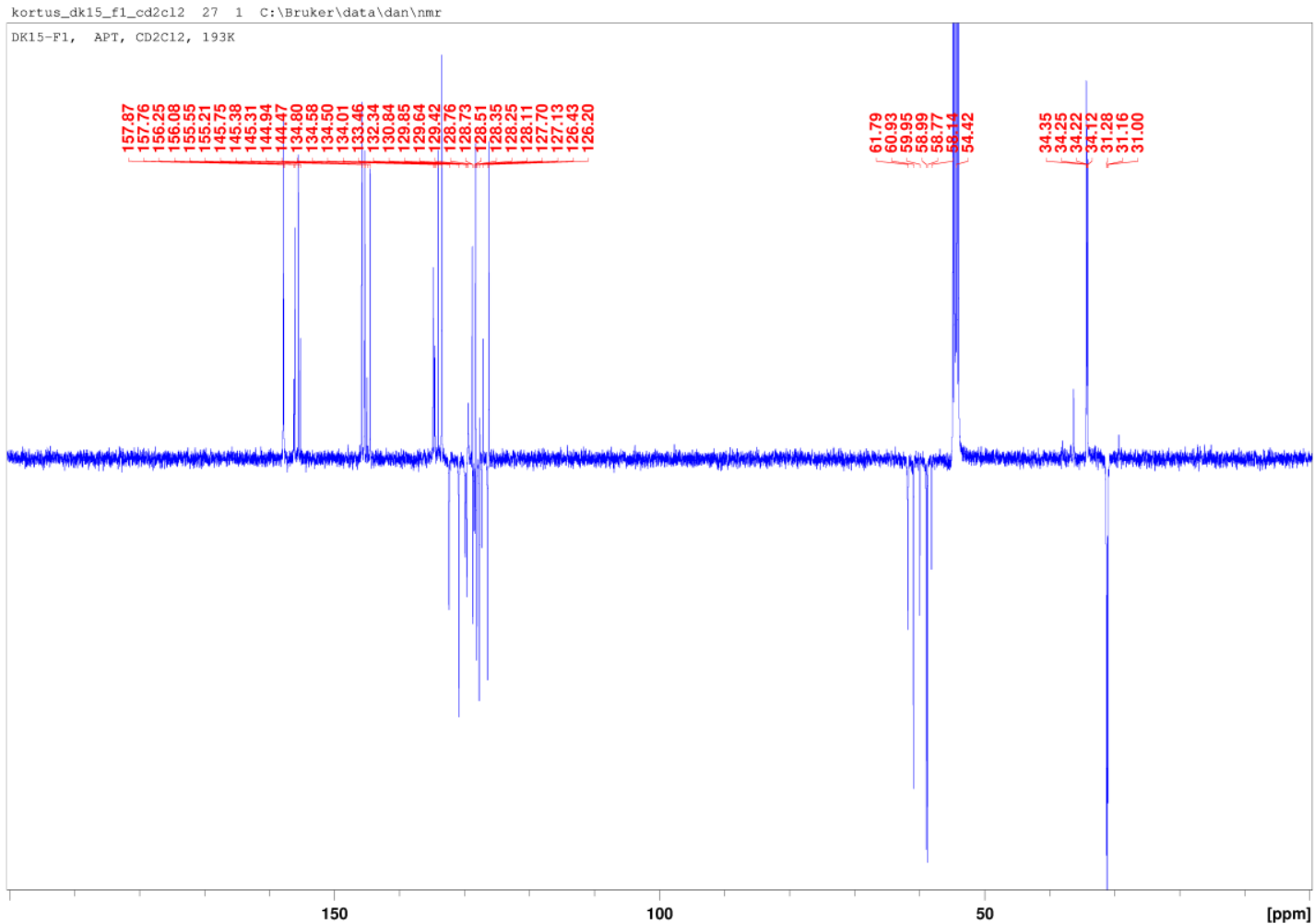

Figure S5: ${ }^{13} \mathrm{C}(\mathrm{APT}) \mathrm{NMR}$ of compound $2\left(125.8 \mathrm{MHz}, \mathrm{CD}_{2} \mathrm{Cl}_{2}, 198 \mathrm{~K}\right)$ 


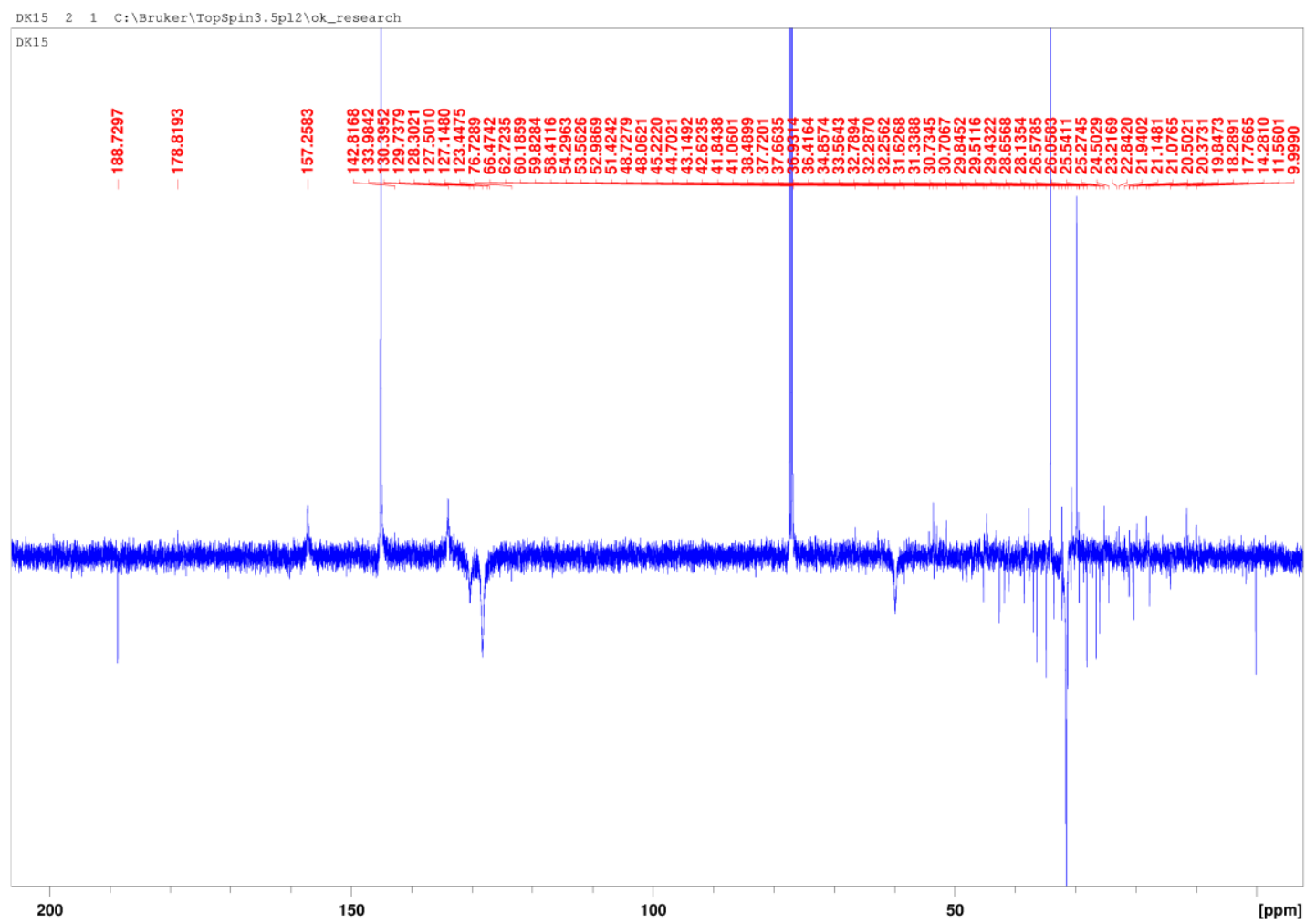

Figure S6: ${ }^{13} \mathrm{C}(\mathrm{APT}) \mathrm{NMR}$ of compound $2\left(100 \mathrm{MHz}, \mathrm{CDCl}_{3}, 298 \mathrm{~K}\right)$ 


\subsection{Compound 3Pr}

262_Kortus_ESIpos_DK57_1 \#42-44- ${ }^{-} \mathrm{RT}^{-}{ }^{-0} 0.61-0.64^{-} \mathrm{AV}^{-}{ }^{-} 3^{-} \mathrm{NL}:{ }^{-} 3.97 \mathrm{E} 7$

T: FTMS + c ESI Fuill ms [2000.00-2000.00]

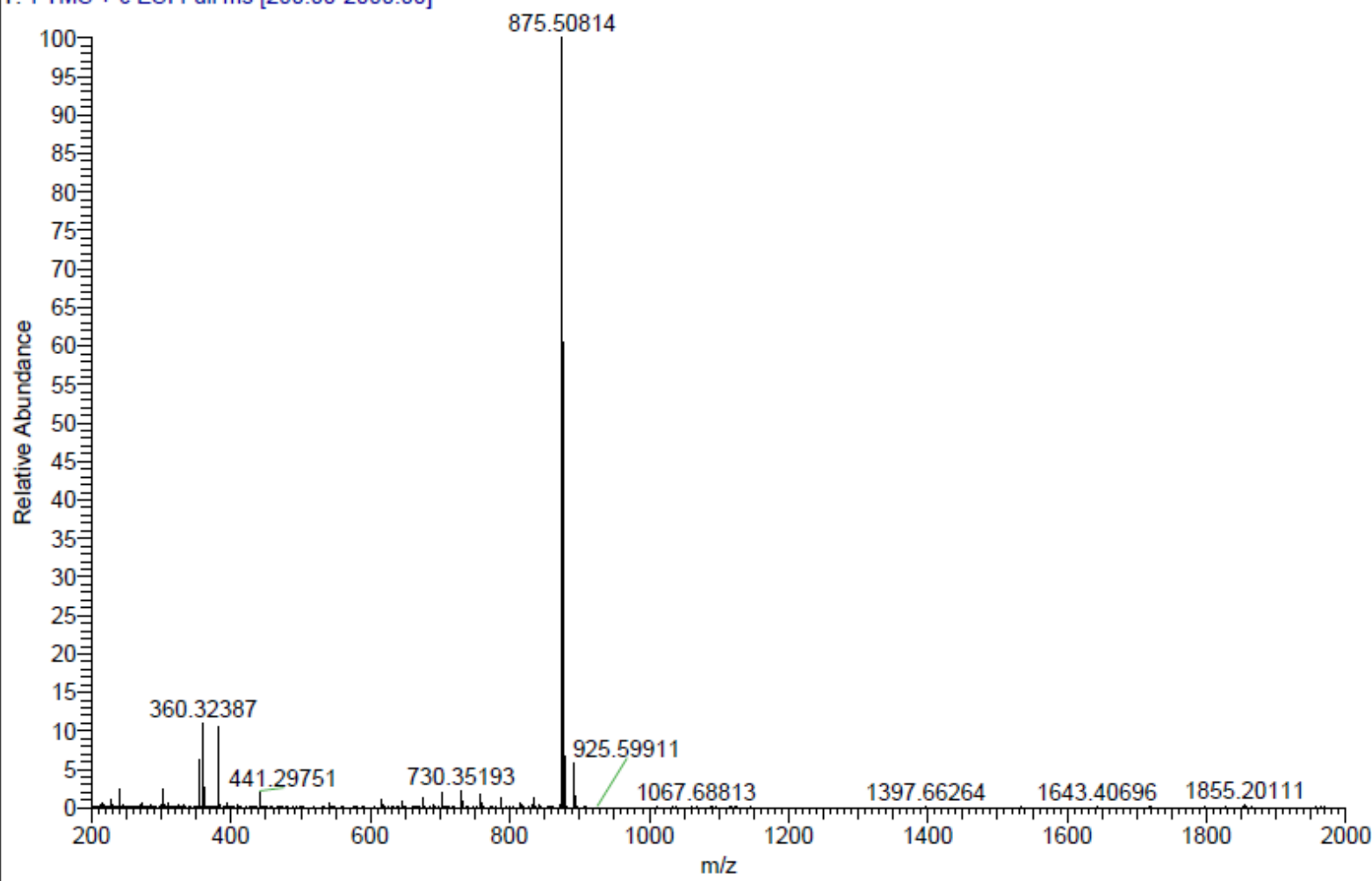

Figure S7: HRMS of compound $3 \operatorname{Pr}\left(\mathrm{ESI}^{+}\right)$

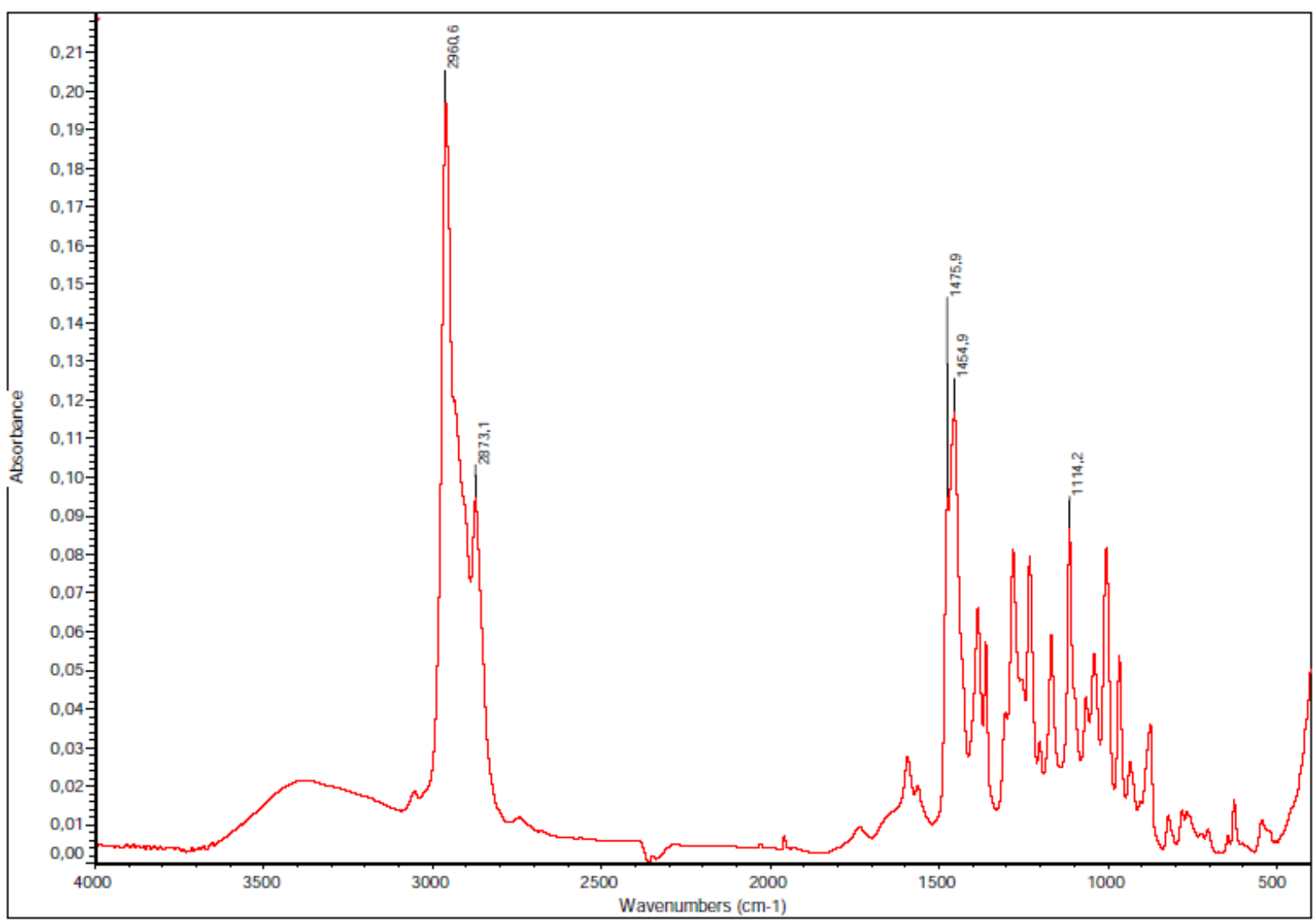

Figure S8: IR of compound 3Pr (KBr) 


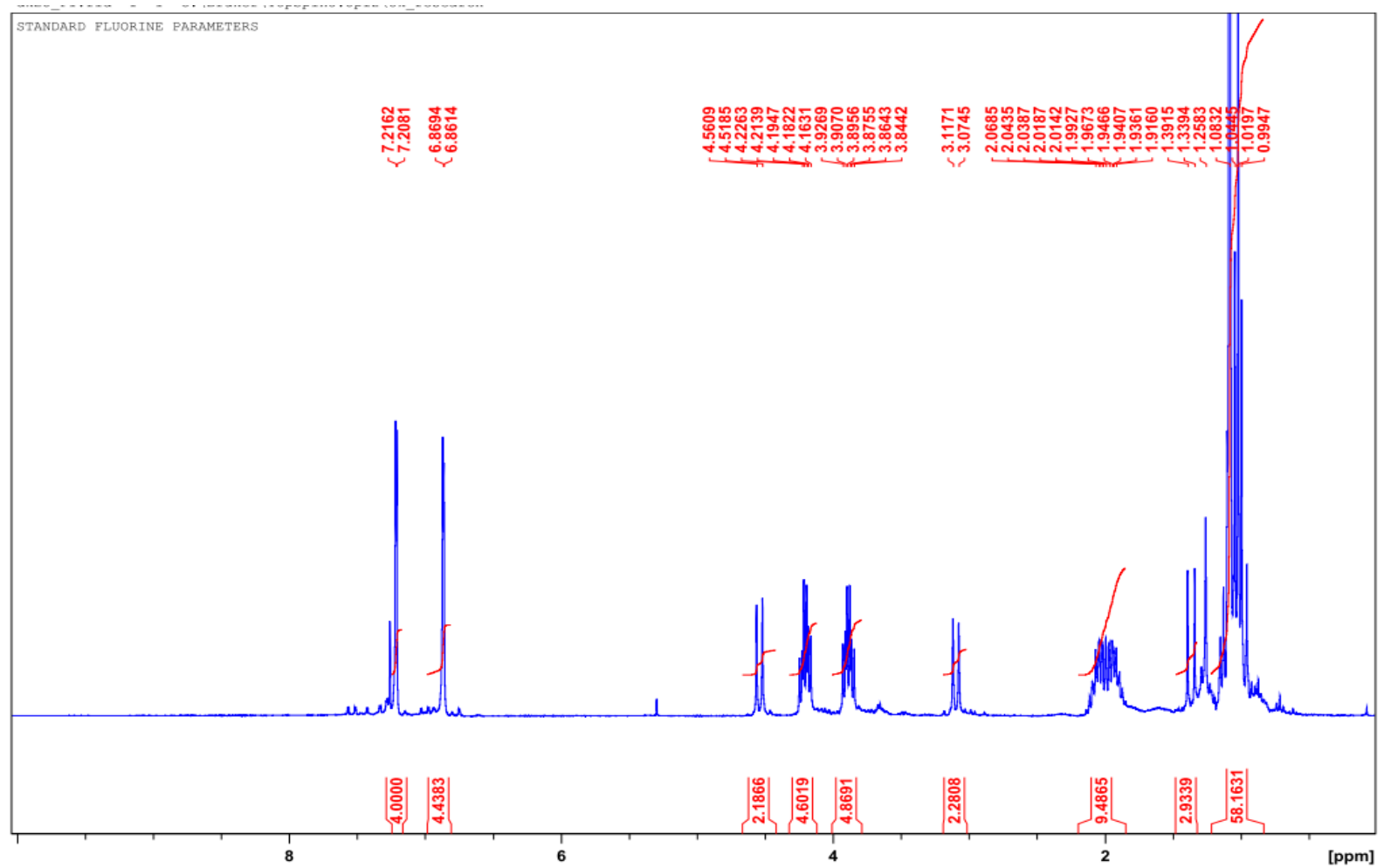

Figure S9: ${ }^{1} \mathrm{H}$ NMR of compound $3 \operatorname{Pr}\left(300 \mathrm{MHz}, \mathrm{CDCl}_{3}, 298 \mathrm{~K}\right)$

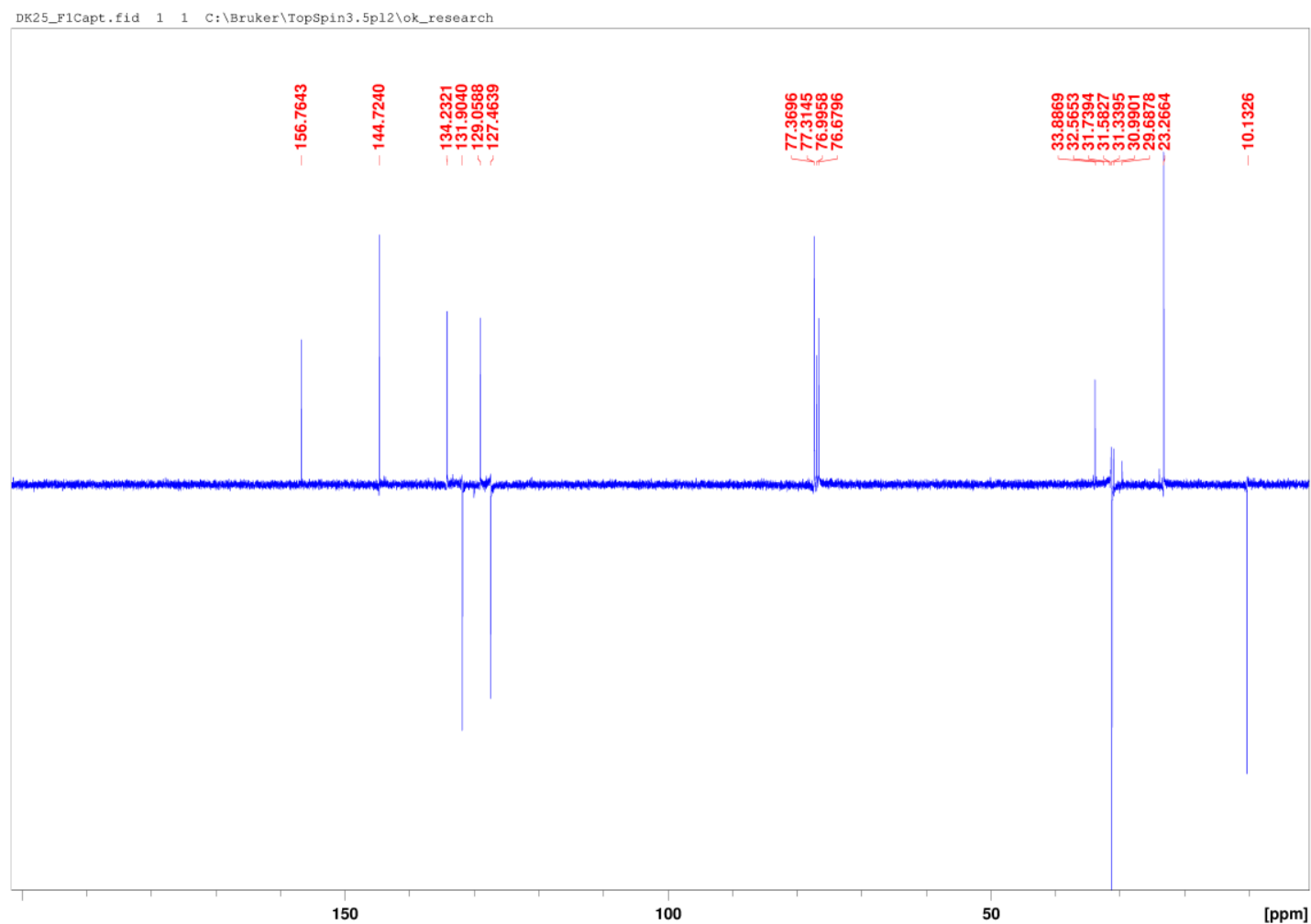

Figure S10: ${ }^{13} \mathrm{C}(\mathrm{APT}) \mathrm{NMR}$ of compound $3 \operatorname{Pr}\left(100 \mathrm{MHz}, \mathrm{CDCl}_{3}, 298 \mathrm{~K}\right)$ 


\subsection{Compound 3Bu}

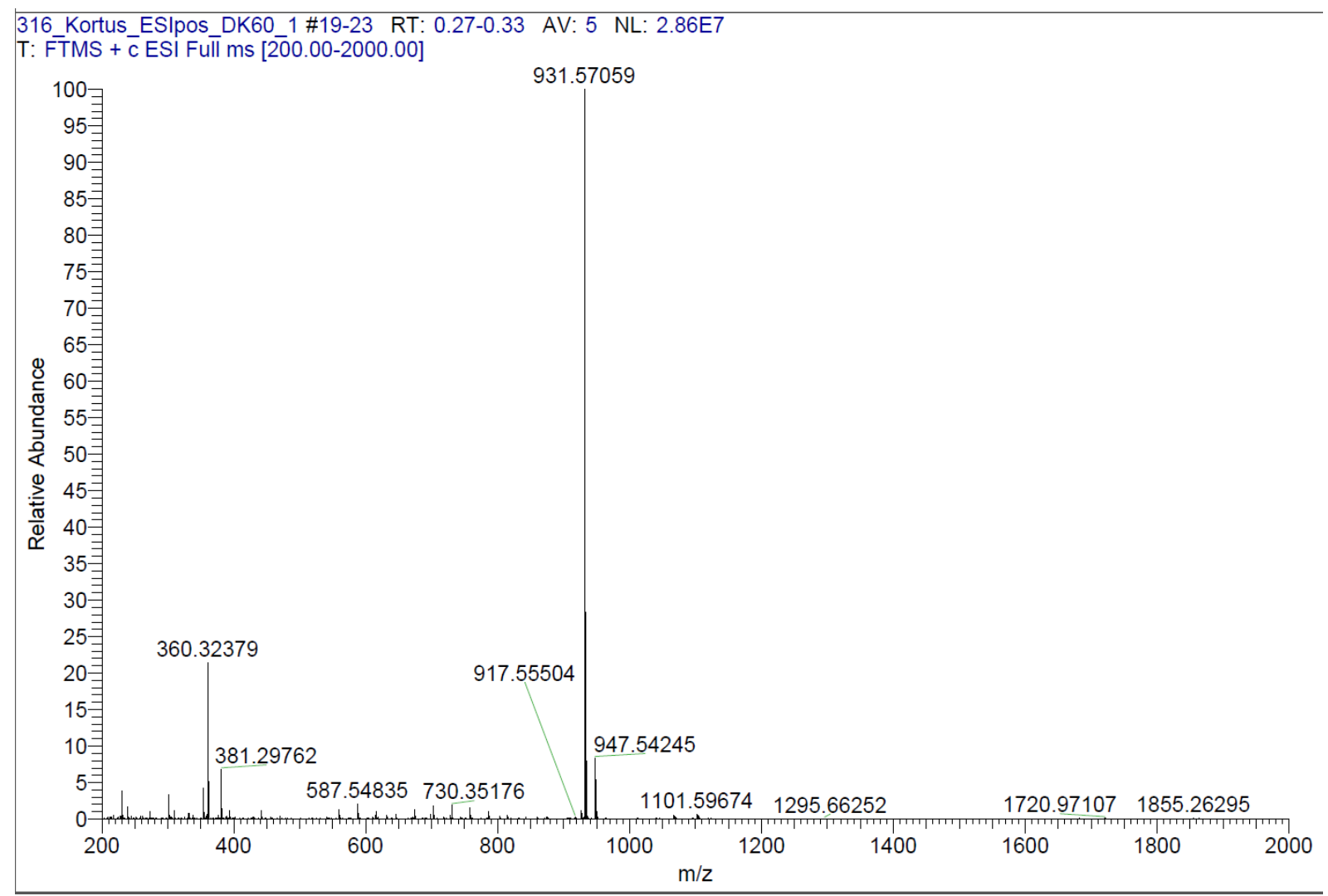

Figure S11: HRMS of compound 3Bu $\left(\mathrm{ESI}^{+}\right)$

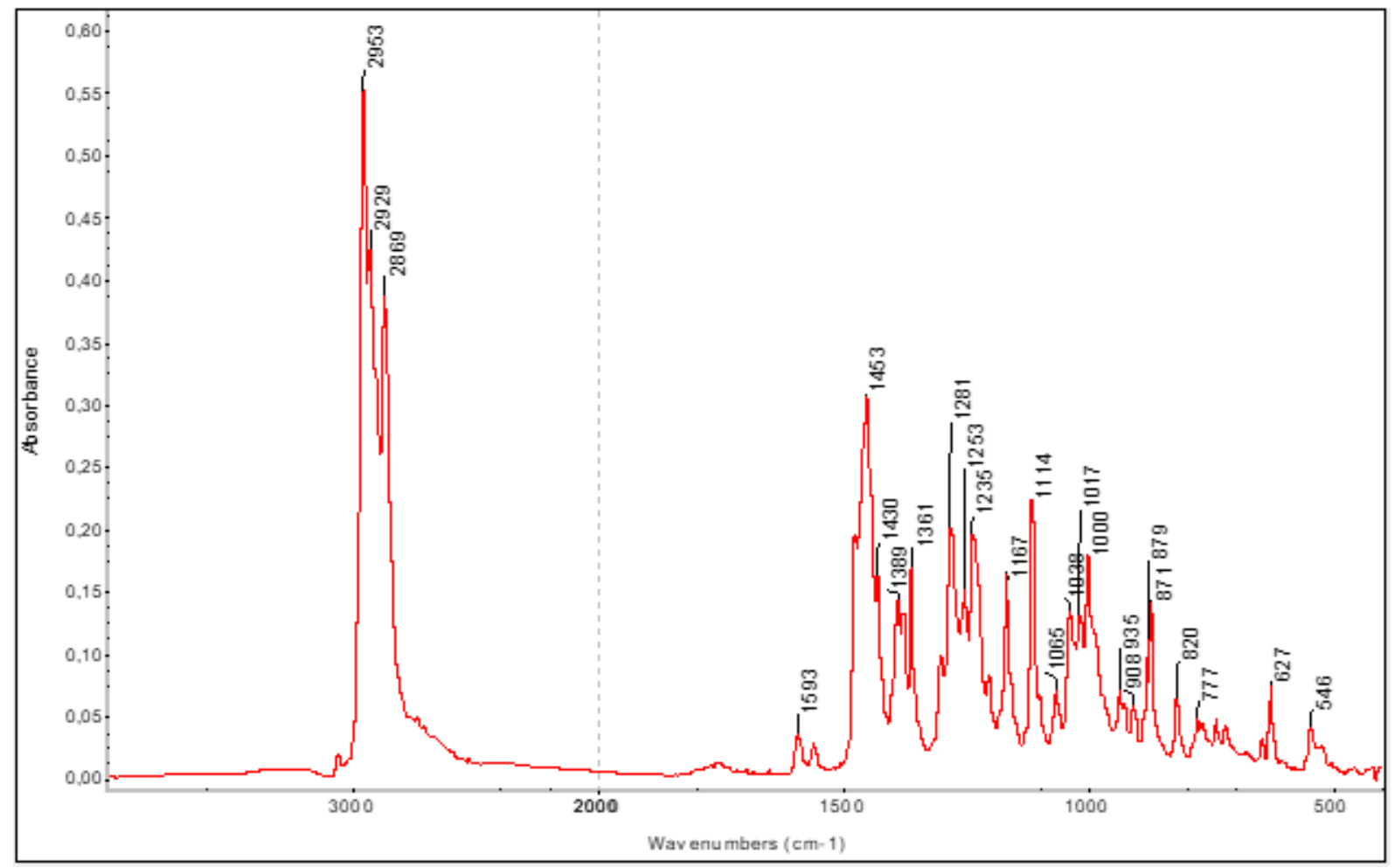

Figure S12: IR of compound $3 \mathrm{Bu}(\mathrm{KBr})$ 


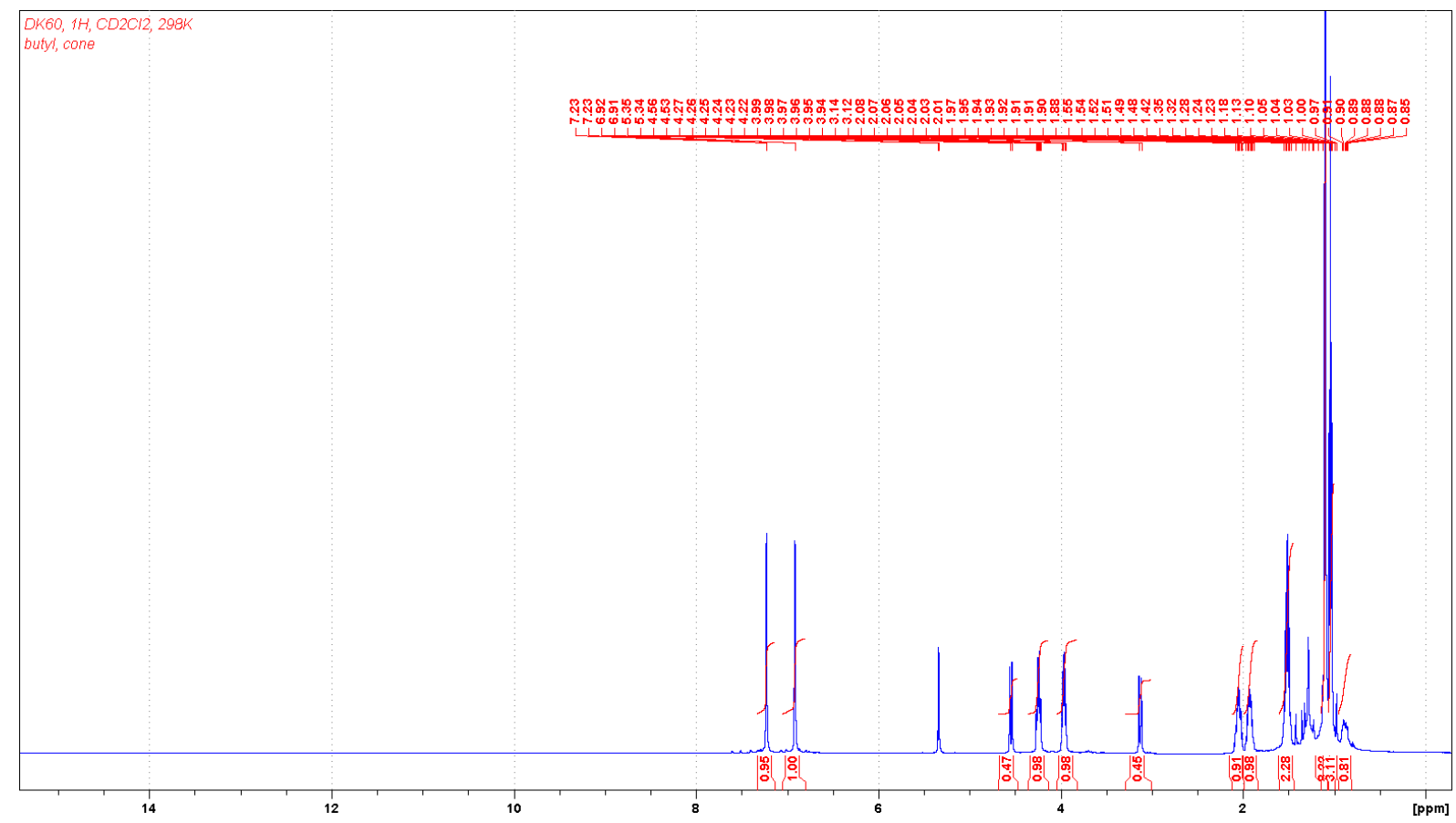

Figure S13: ${ }^{1} \mathrm{H}$ NMR of compound 3Bu $\left(500.1 \mathrm{MHz}, \mathrm{CD}_{2} \mathrm{Cl}_{2}, 298 \mathrm{~K}\right)$

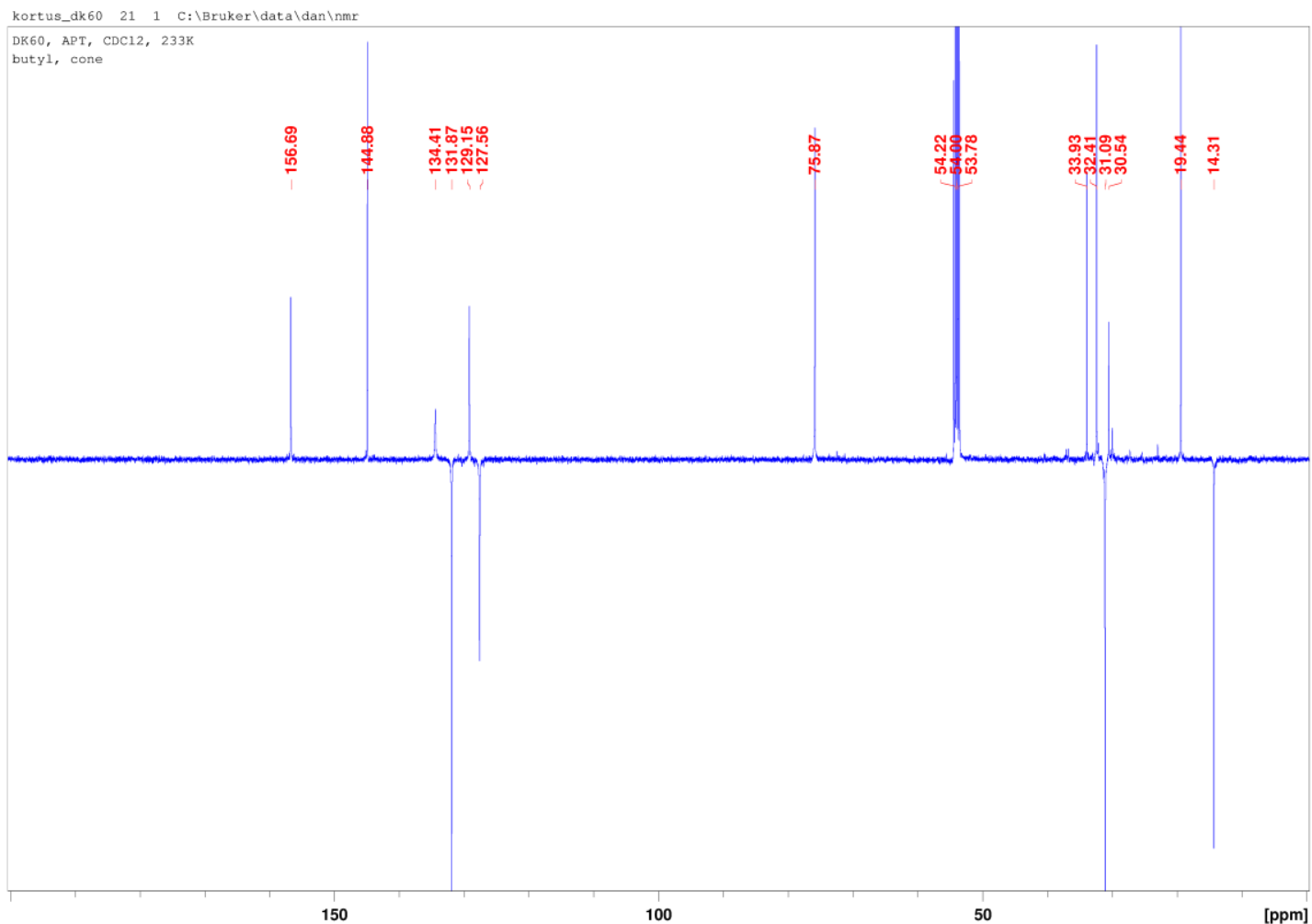

Figure S14: ${ }^{13} \mathrm{C}$ (APT) NMR of compound 3Bu $\left(125.8 \mathrm{MHz}, \mathrm{CDCl}_{3}, 233 \mathrm{~K}\right)$ 


\subsection{Compound 4Et}

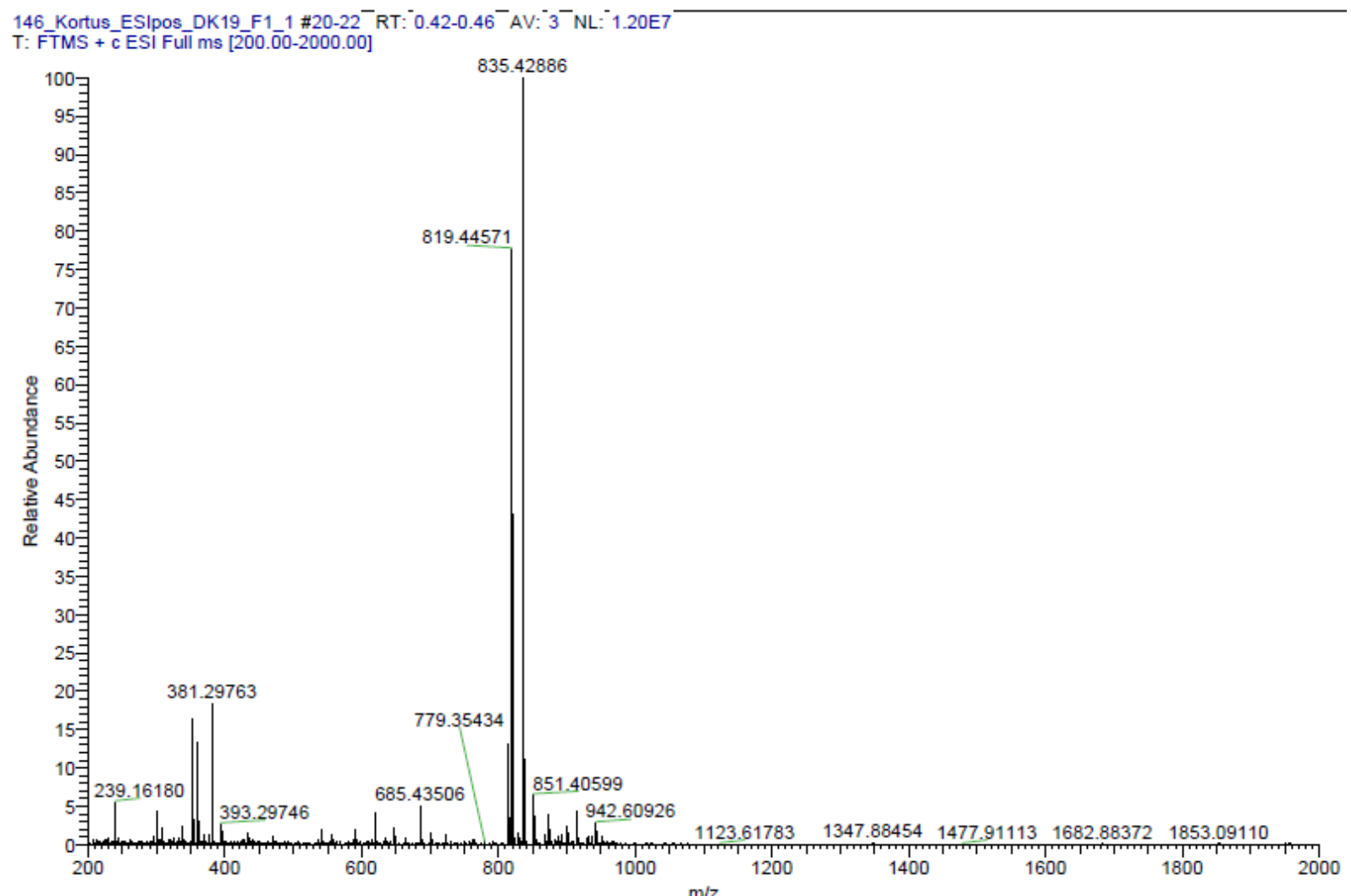

Figure S15: HRMS of compound 4Et $\left(\mathrm{ESI}^{+}\right)$

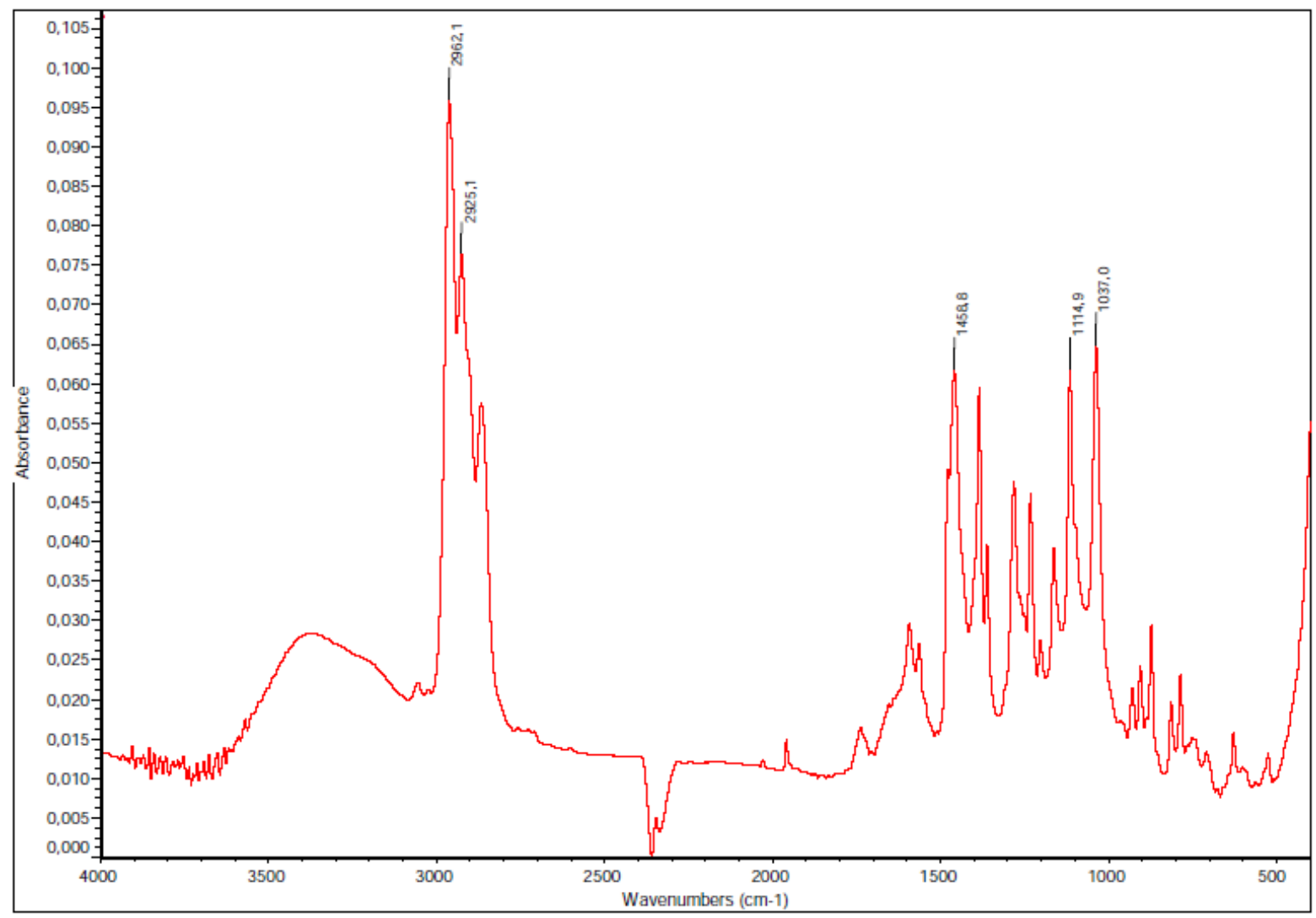

Figure S16: IR of compound 4Et (KBr) 


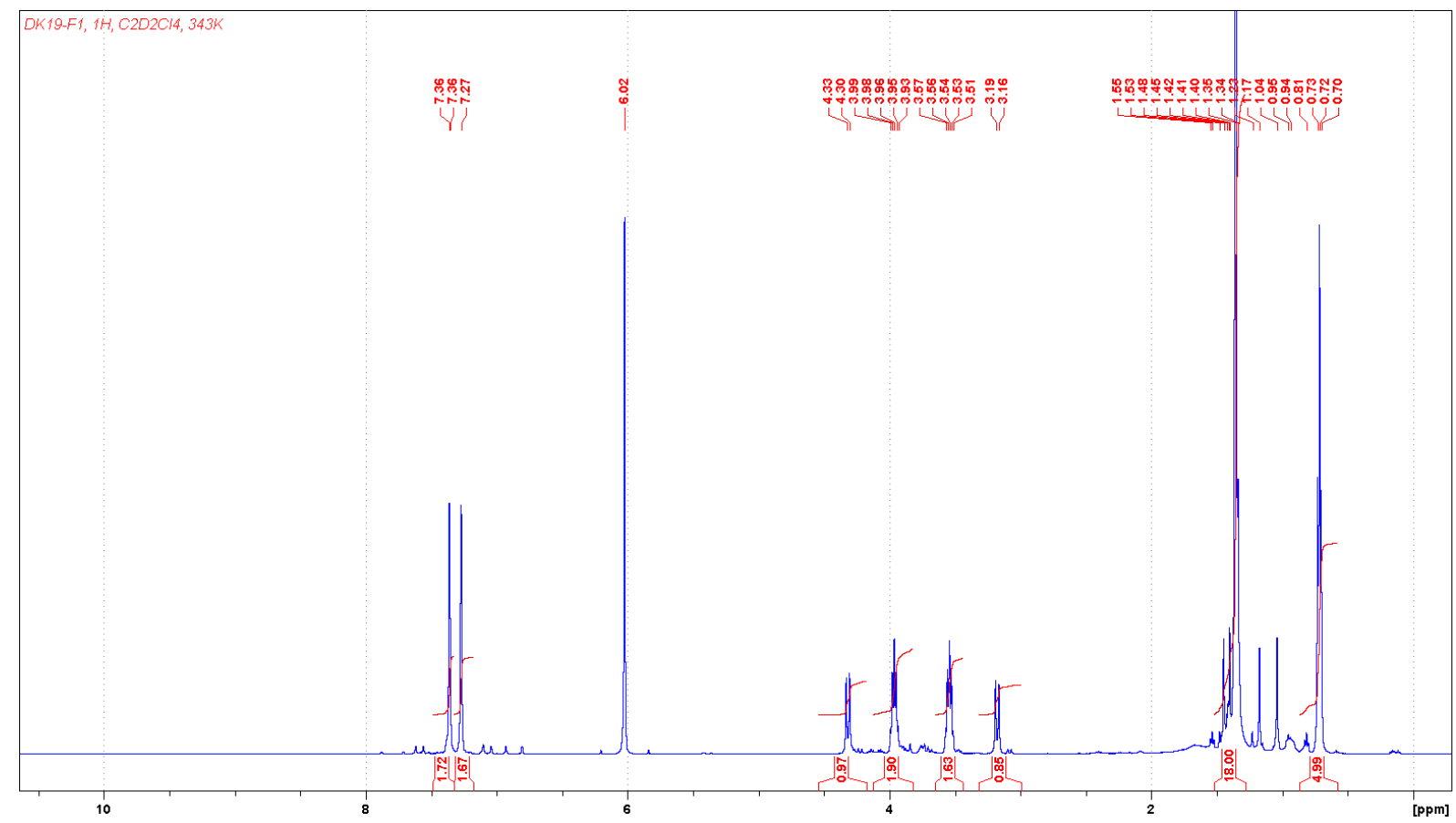

Figure S17: ${ }^{1} \mathrm{H}$ NMR of compound $4 \mathrm{Et}\left(500.1 \mathrm{MHz}, \mathrm{C}_{2} \mathrm{D}_{2} \mathrm{Cl}_{4}, 343 \mathrm{~K}\right)$

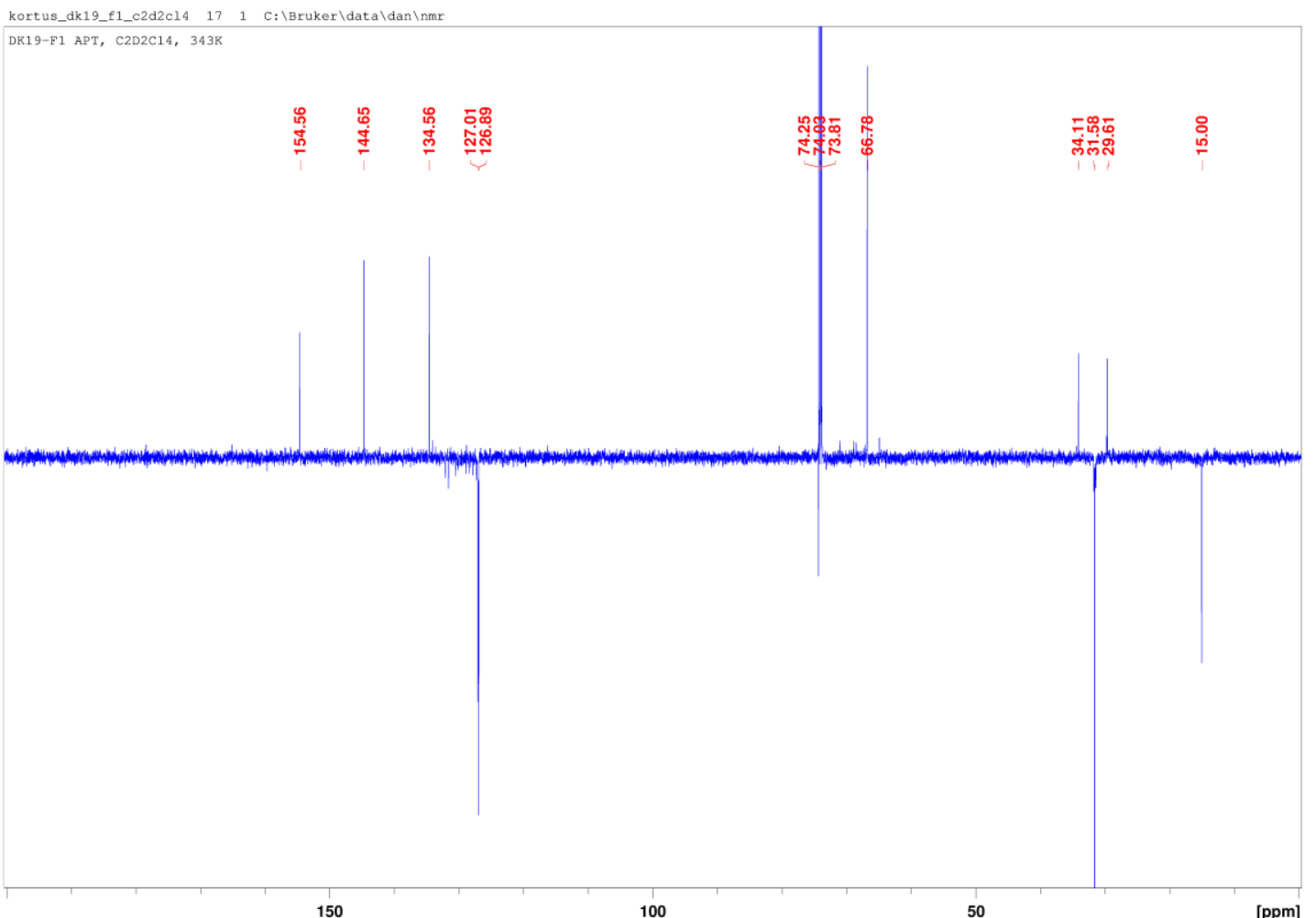

Figure S18: ${ }^{13} \mathrm{C}$ (APT) NMR of compound 4Et (125.8 MHz, $\left.\mathrm{C}_{2} \mathrm{D}_{2} \mathrm{Cl}_{4}, 343 \mathrm{~K}\right)$ 


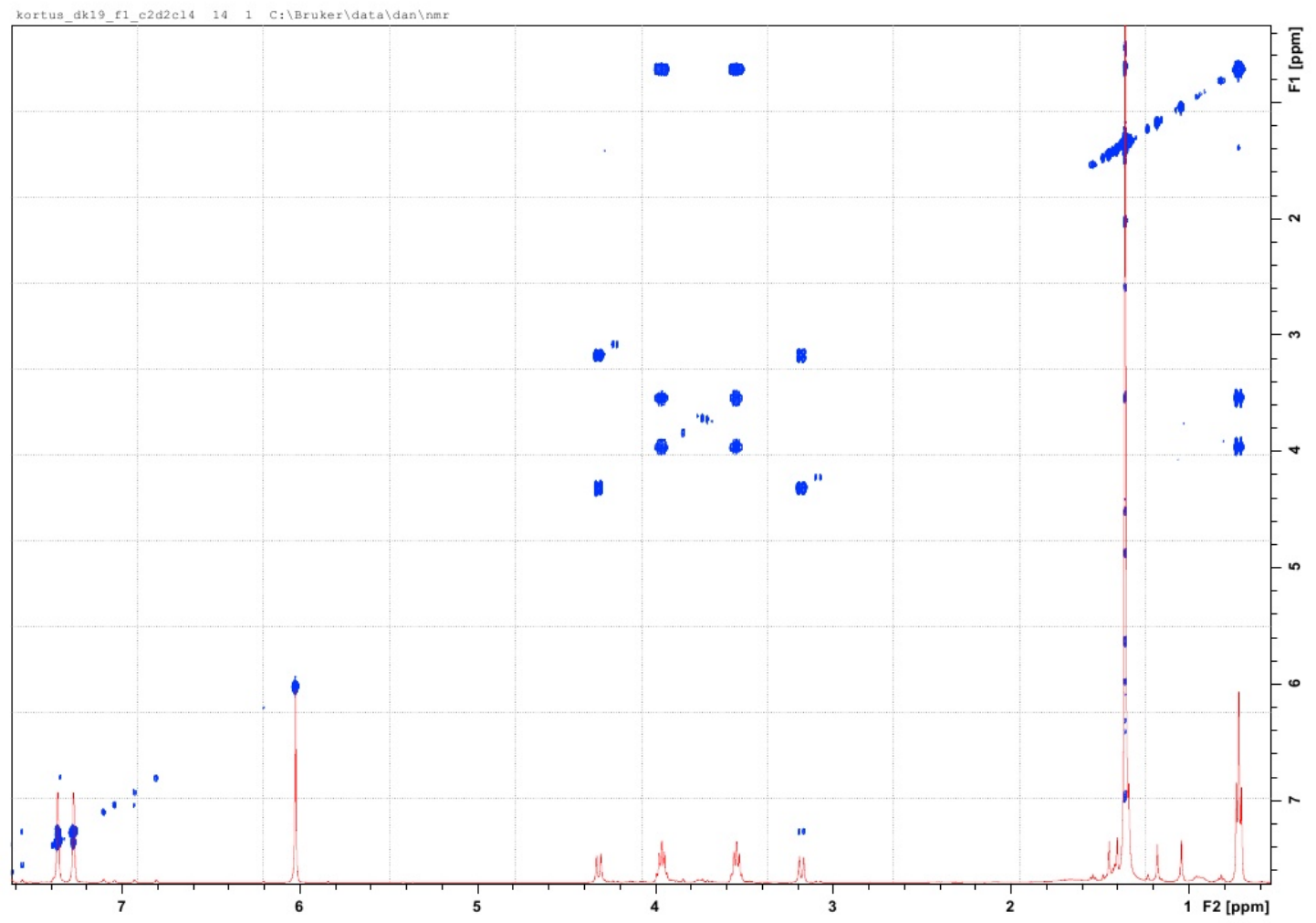

Figure S19: ${ }^{1} \mathrm{H}-{ }^{1} \mathrm{H}$ COSY NMR of compound $4 \mathrm{Et}\left(500 \mathrm{MHz}, \mathrm{C}_{2} \mathrm{D}_{2} \mathrm{Cl}_{4}\right)$

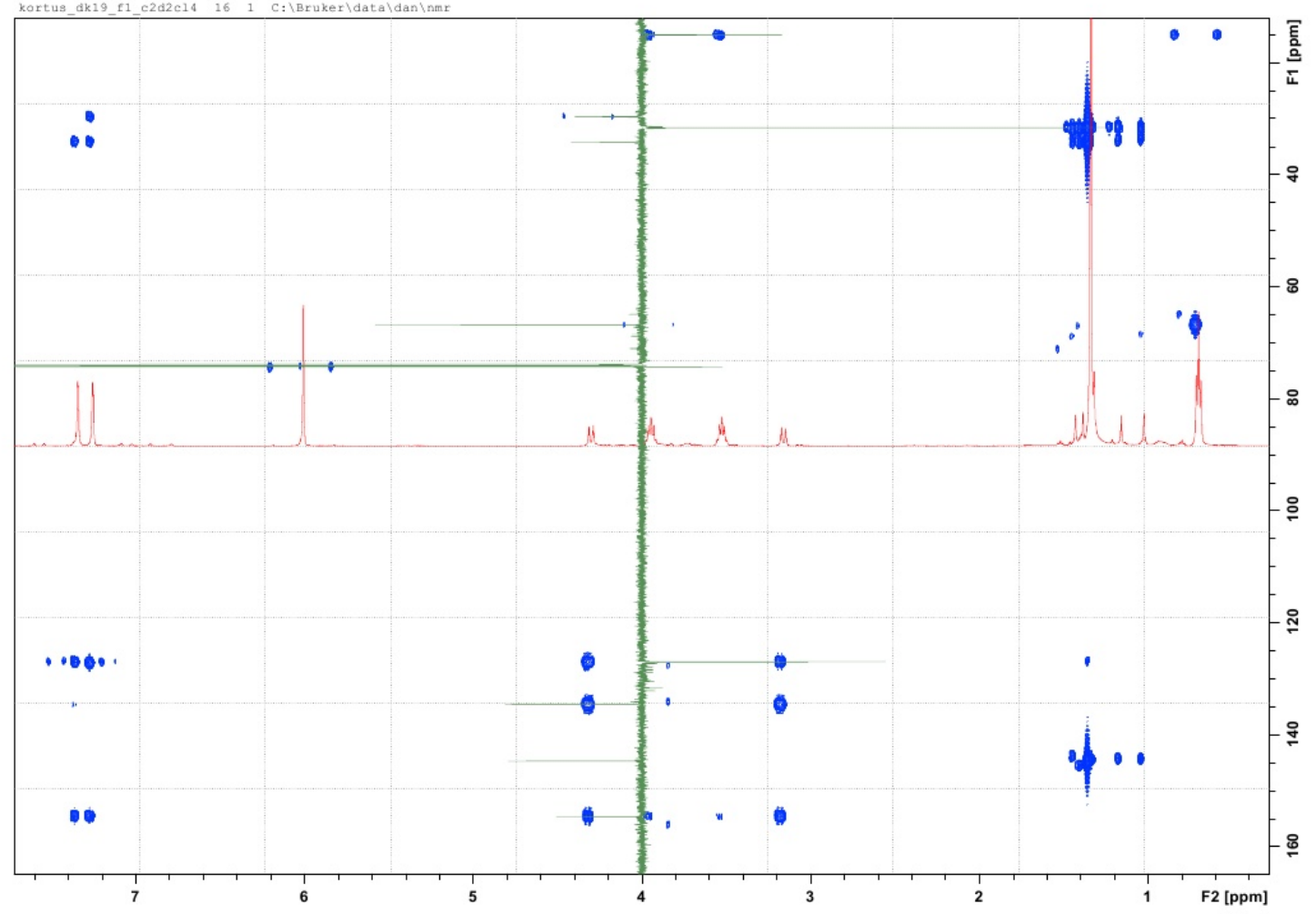

Figure S20: $\mathrm{HMBC}$ NMR of compound 4Et $\left(500 \mathrm{MHz}, \mathrm{C}_{2} \mathrm{D}_{2} \mathrm{Cl}_{4}\right)$ 


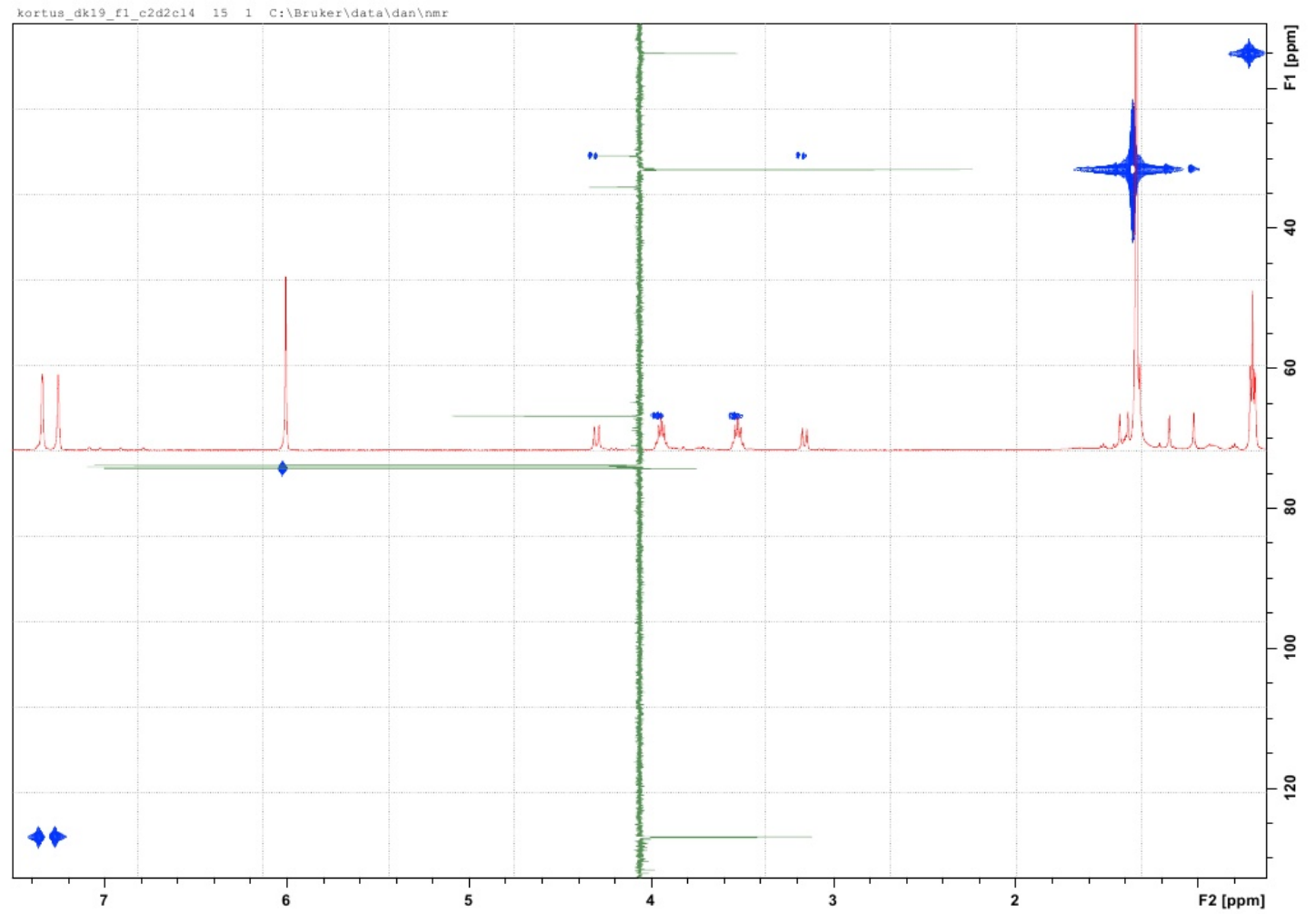

Figure S21: HMQC NMR of compound 4Et (500 MHz, $\left.\mathrm{C}_{2} \mathrm{D}_{2} \mathrm{Cl}_{4}\right)$ 


\subsection{Compound 4Pr}

155_Kortus_ESIpos_DK58_1 \#52-56 RT: 0.75-0.81 AV: 5 NL: $2.82 E 7$

T: FTMS + c ESI Full ms [200.00-2000.00]

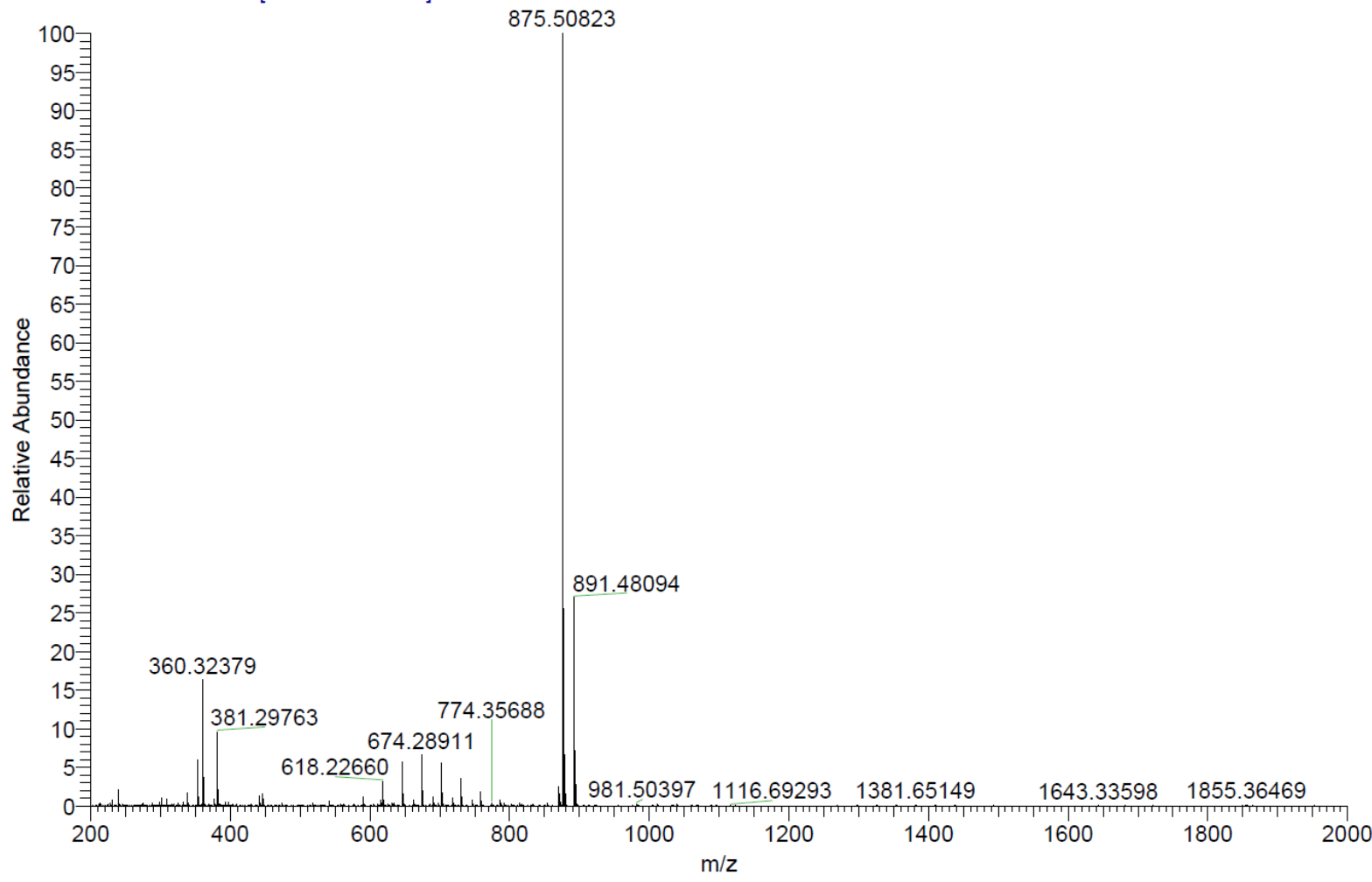

Figure S22: HRMS of compound $4 \operatorname{Pr}\left(\mathrm{ESI}^{+}\right)$

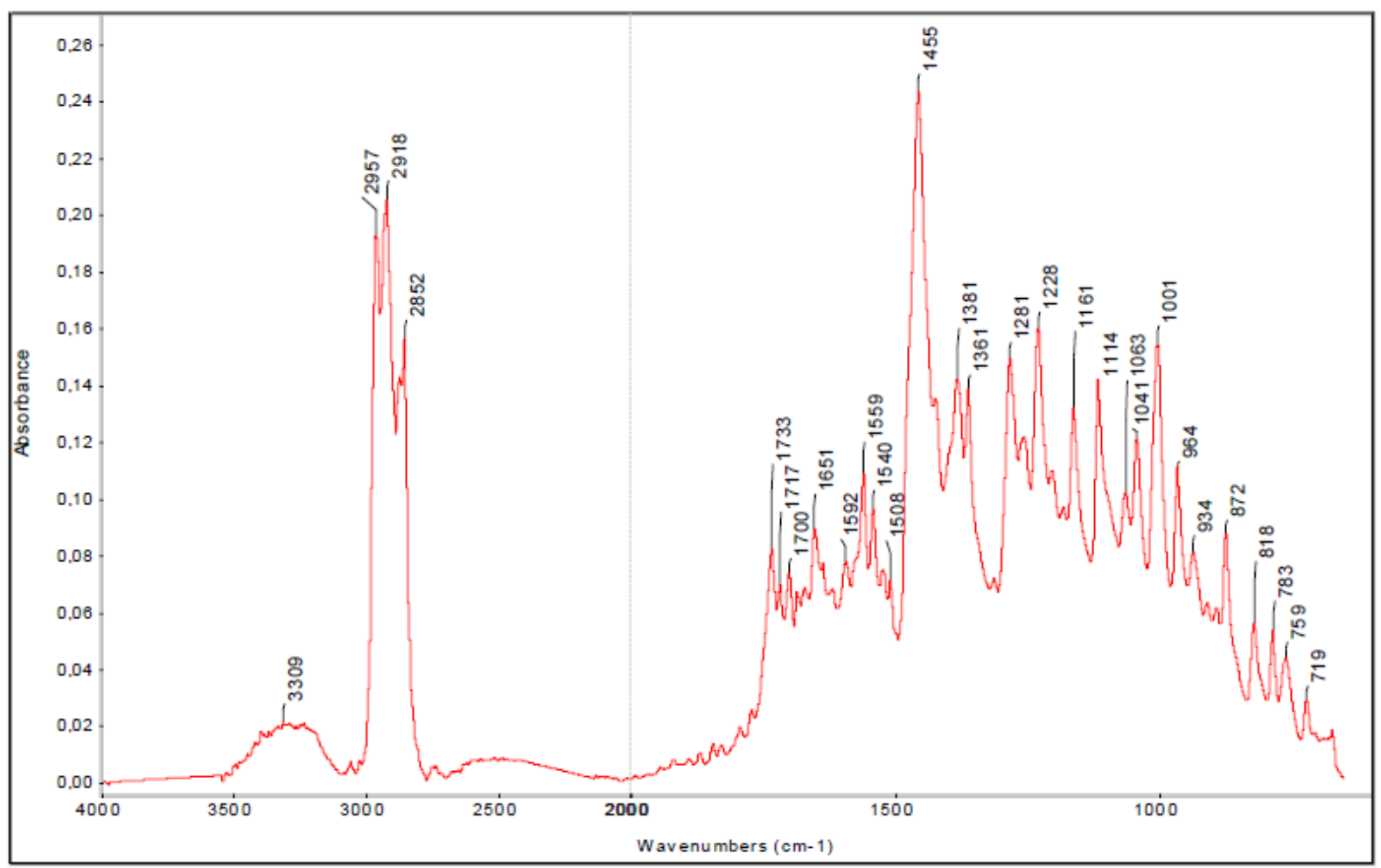

Figure S23: IR of compound $4 \mathrm{Pr}(\mathrm{KBr})$ 


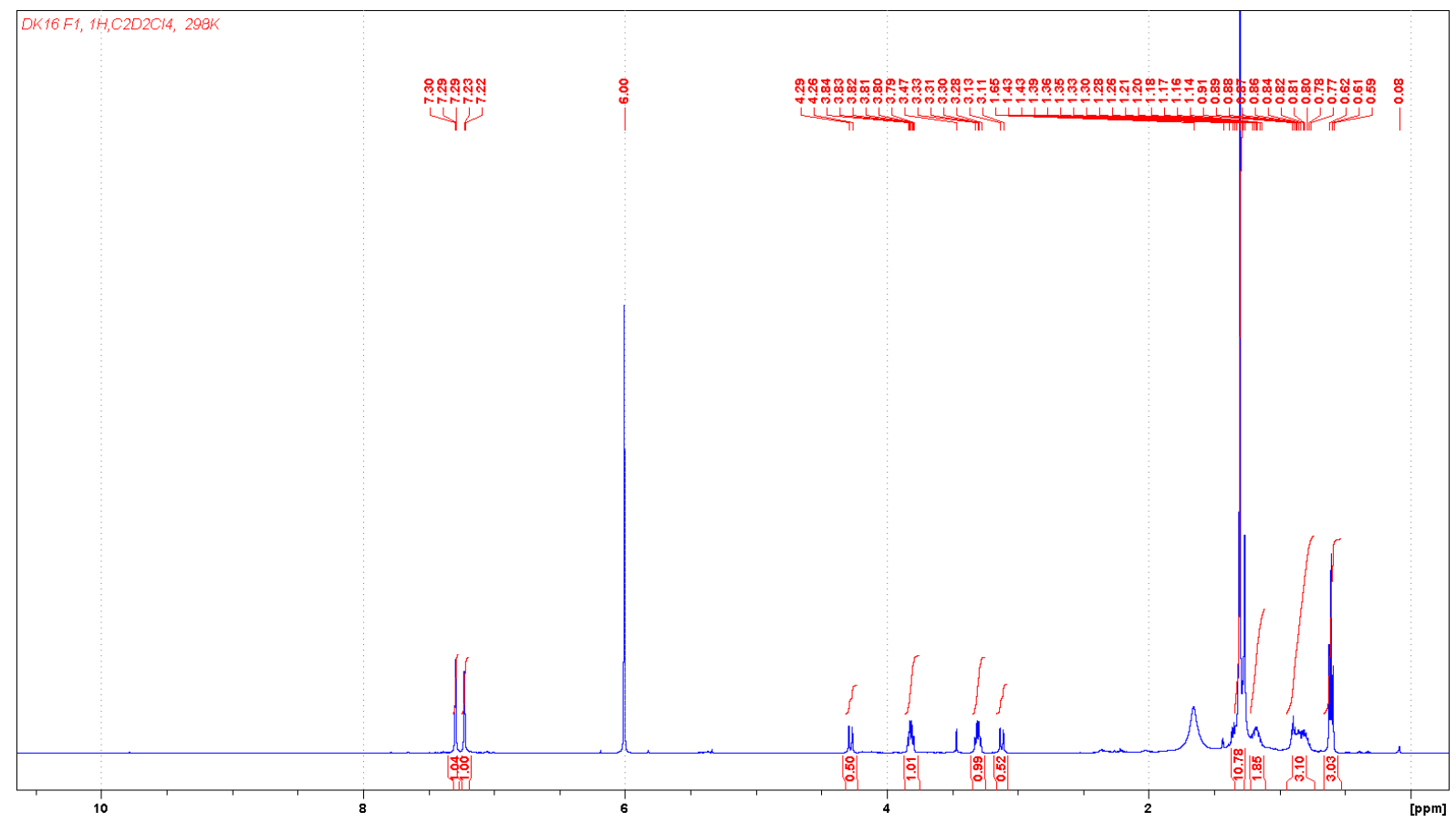

Figure S24: ${ }^{1} \mathrm{H} N M R$ of compound $4 \operatorname{Pr}\left(600.1 \mathrm{MHz}, \mathrm{C}_{2} \mathrm{D}_{2} \mathrm{Cl}_{4}, 298 \mathrm{~K}\right)$

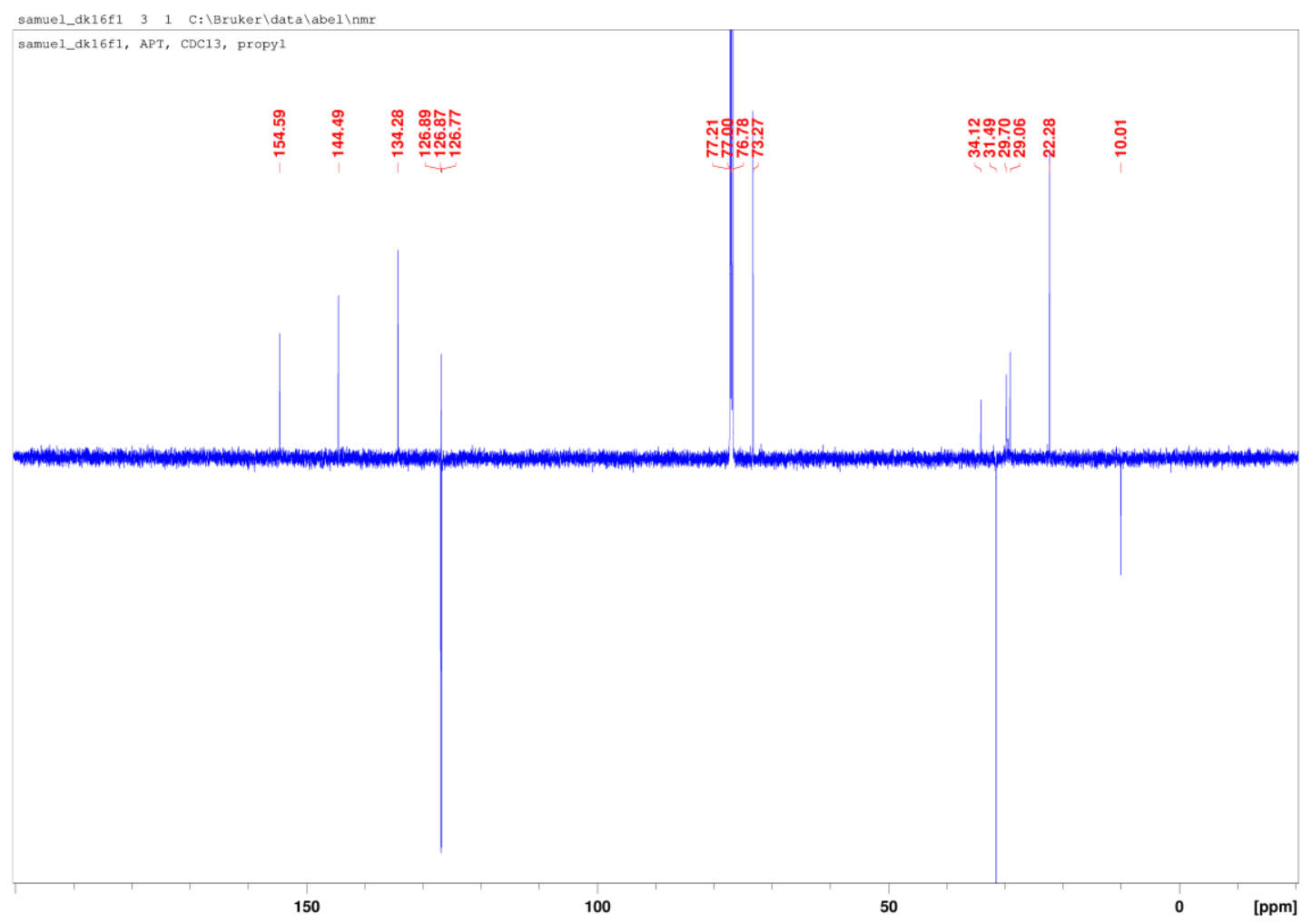

Figure S25: ${ }^{13} \mathrm{C}(\mathrm{APT}) \mathrm{NMR}$ of compound $4 \operatorname{Pr}\left(150.9 \mathrm{MHz}, \mathrm{CDCl}_{3}\right)$ 


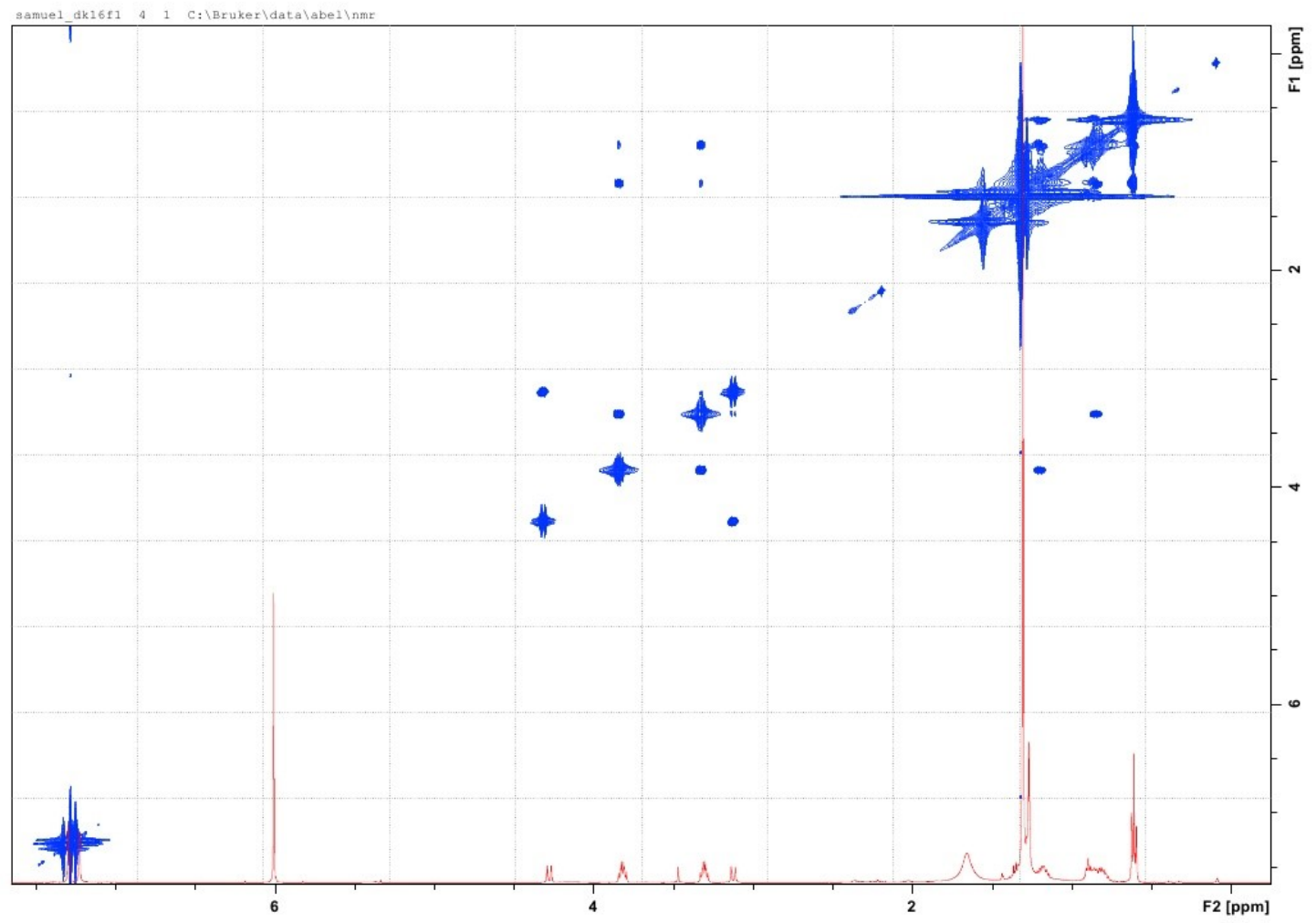

Figure S26: ${ }^{1} \mathrm{H}-{ }^{1} \mathrm{H}$ COSY NMR of compound $4 \operatorname{Pr}\left(600 \mathrm{MHz}, \mathrm{C}_{2} \mathrm{D}_{2} \mathrm{Cl}_{4}\right)$

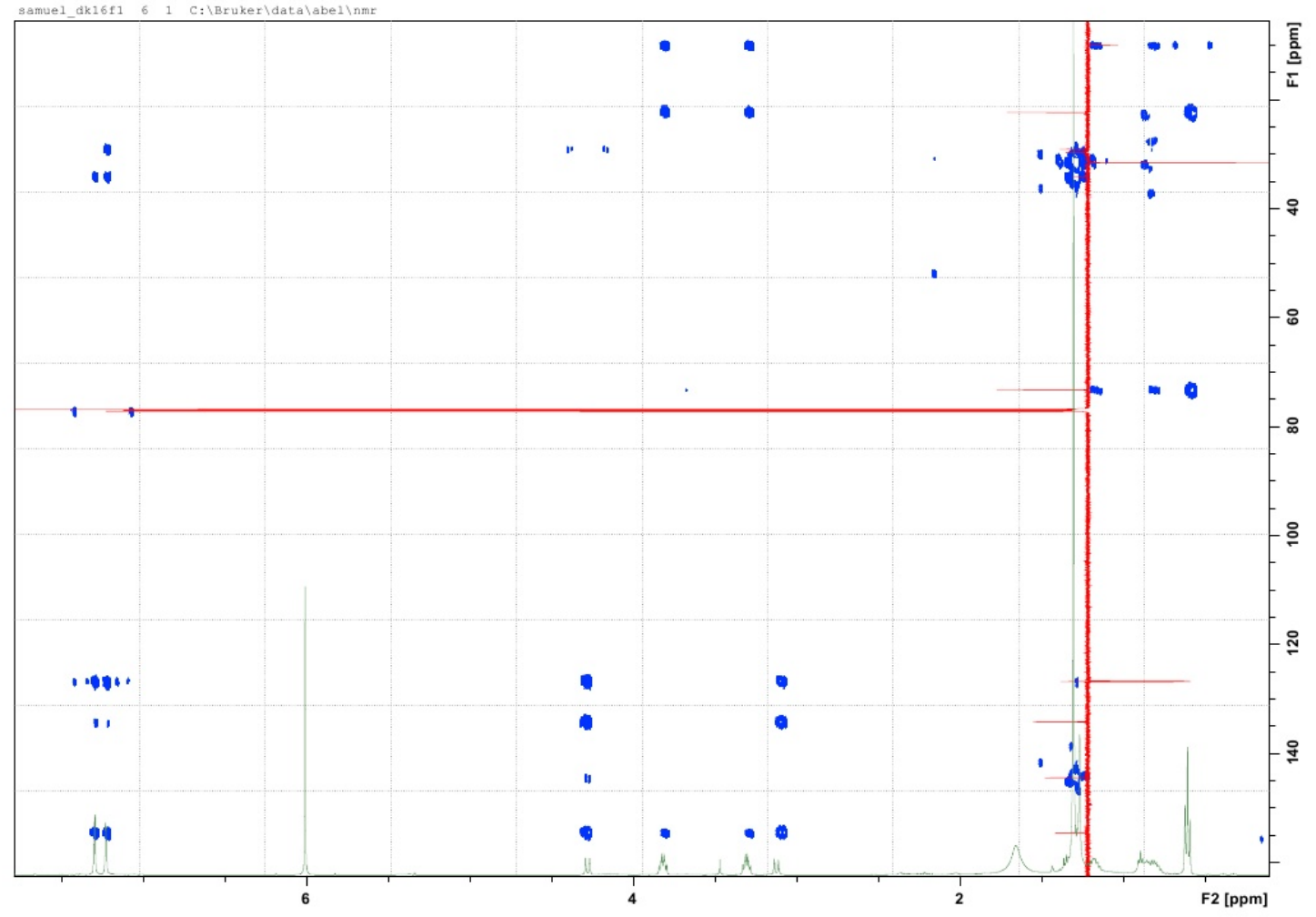

Figure S27: $\mathrm{HMBC} N M R$ of compound $4 \operatorname{Pr}\left(600 \mathrm{MHz}, \mathrm{C}_{2} \mathrm{D}_{2} \mathrm{Cl}_{4}\right)$ 


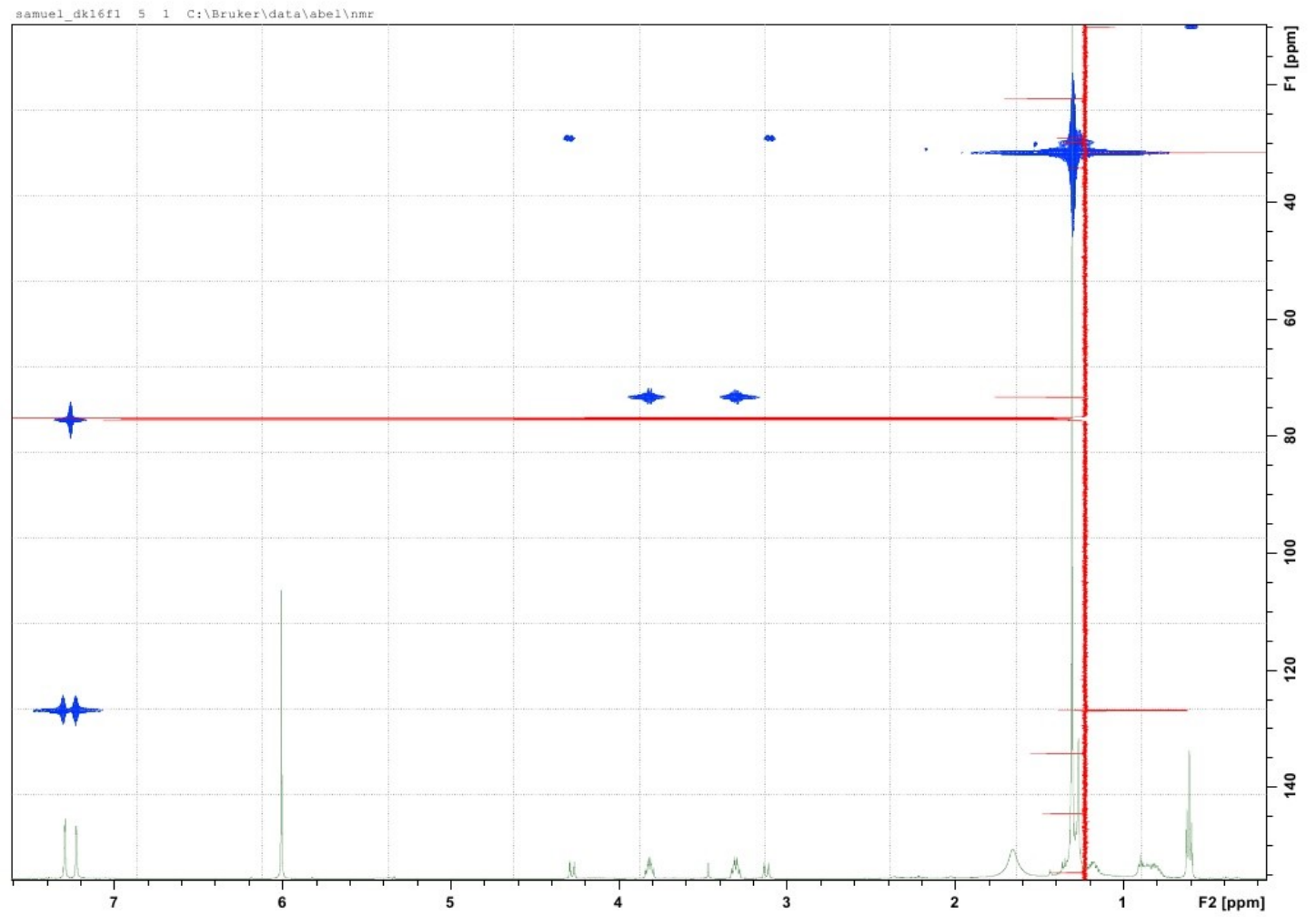

Figure S28: $\mathrm{HMQC}$ NMR of compound $4 \operatorname{Pr}\left(600 \mathrm{MHz}, \mathrm{C}_{2} \mathrm{D}_{2} \mathrm{Cl}_{4}\right)$ 


\subsection{Compound 4Bu}

316_Kortus_ESIpos_DK59_1_1 \#46-50 RT: 0.67-0.73 AV: 5 NL: 2.41E7

T: FTMS + c ESI Full ms [200.00-2000.00]

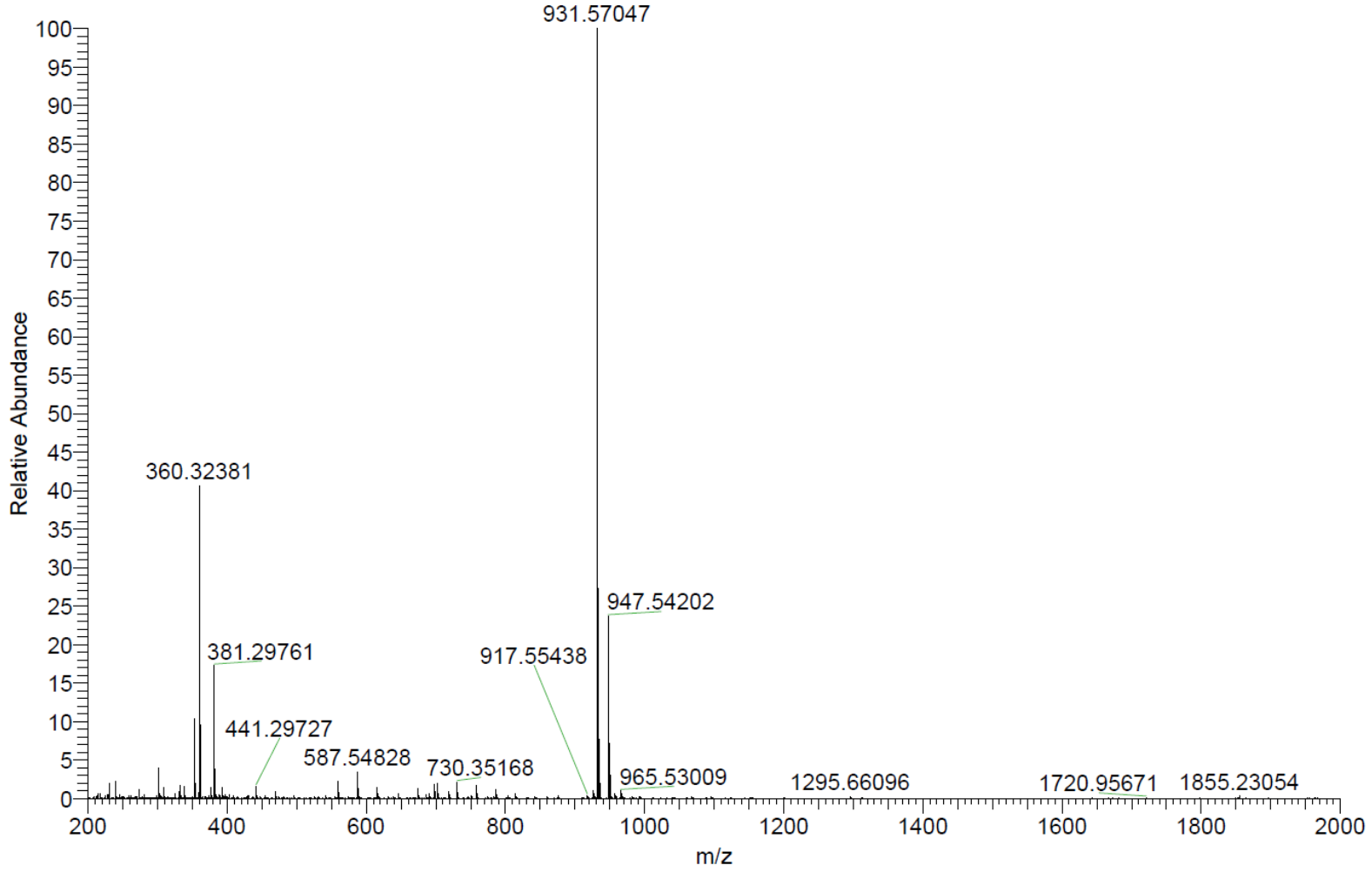

Figure S29: HRMS of compound 4Bu (ESI $\left.{ }^{+}\right)$

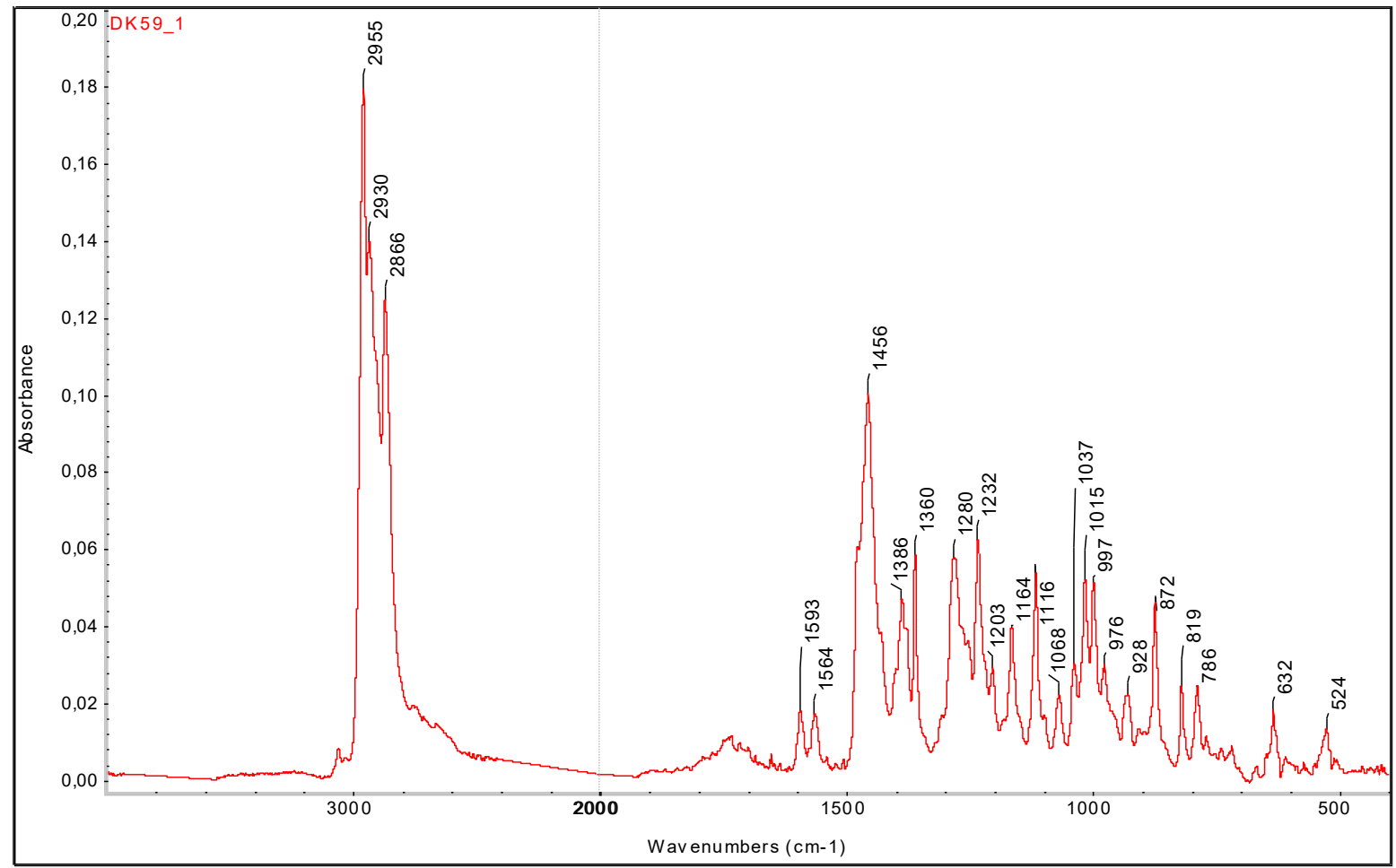

Figure S30: IR of compound 4Bu (KBr) 


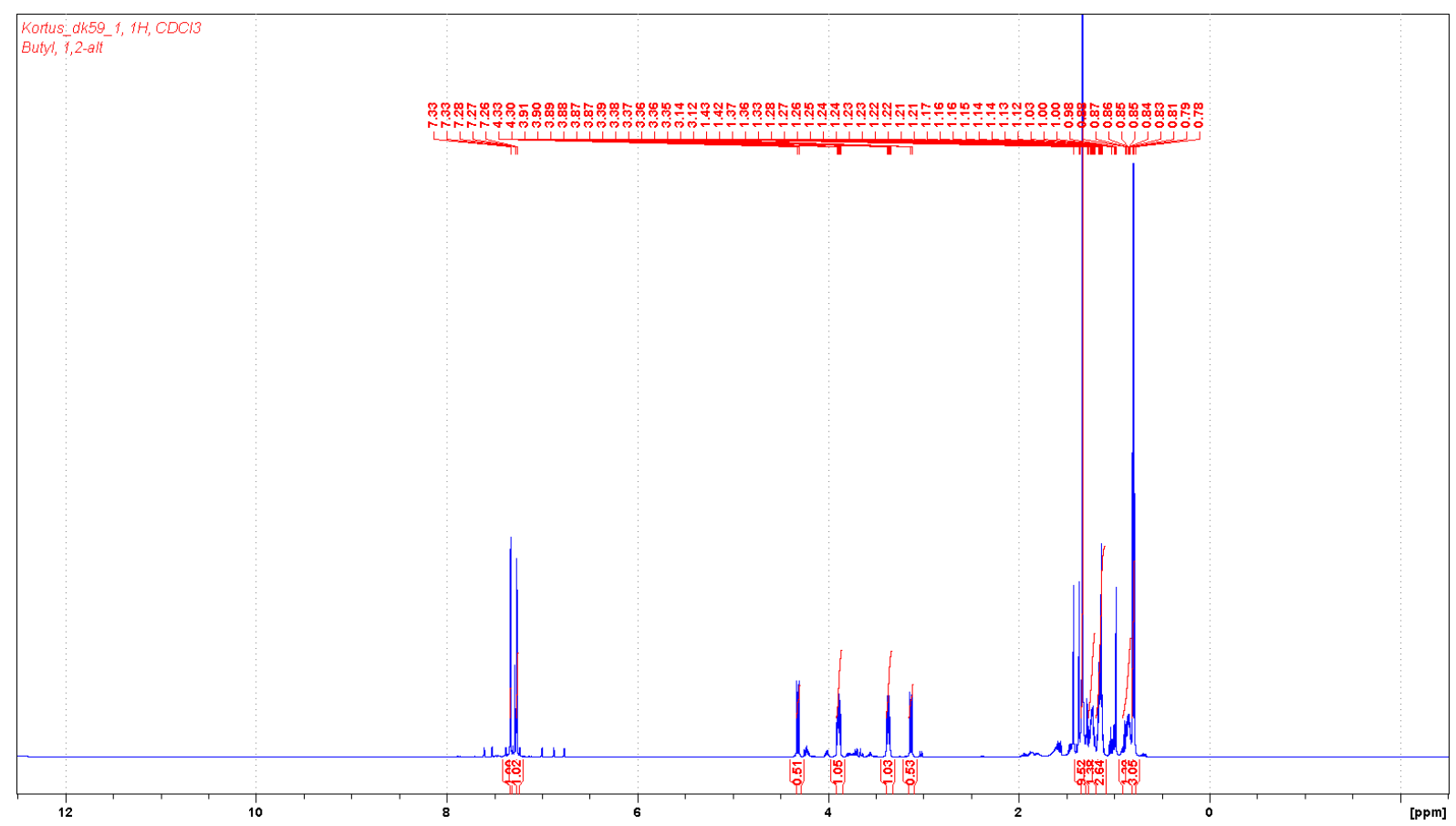

Figure S31: ${ }^{1} \mathrm{H}$ NMR of compound $4 \mathrm{Bu}\left(600.1 \mathrm{MHz}, \mathrm{CDCl}_{3}\right)$
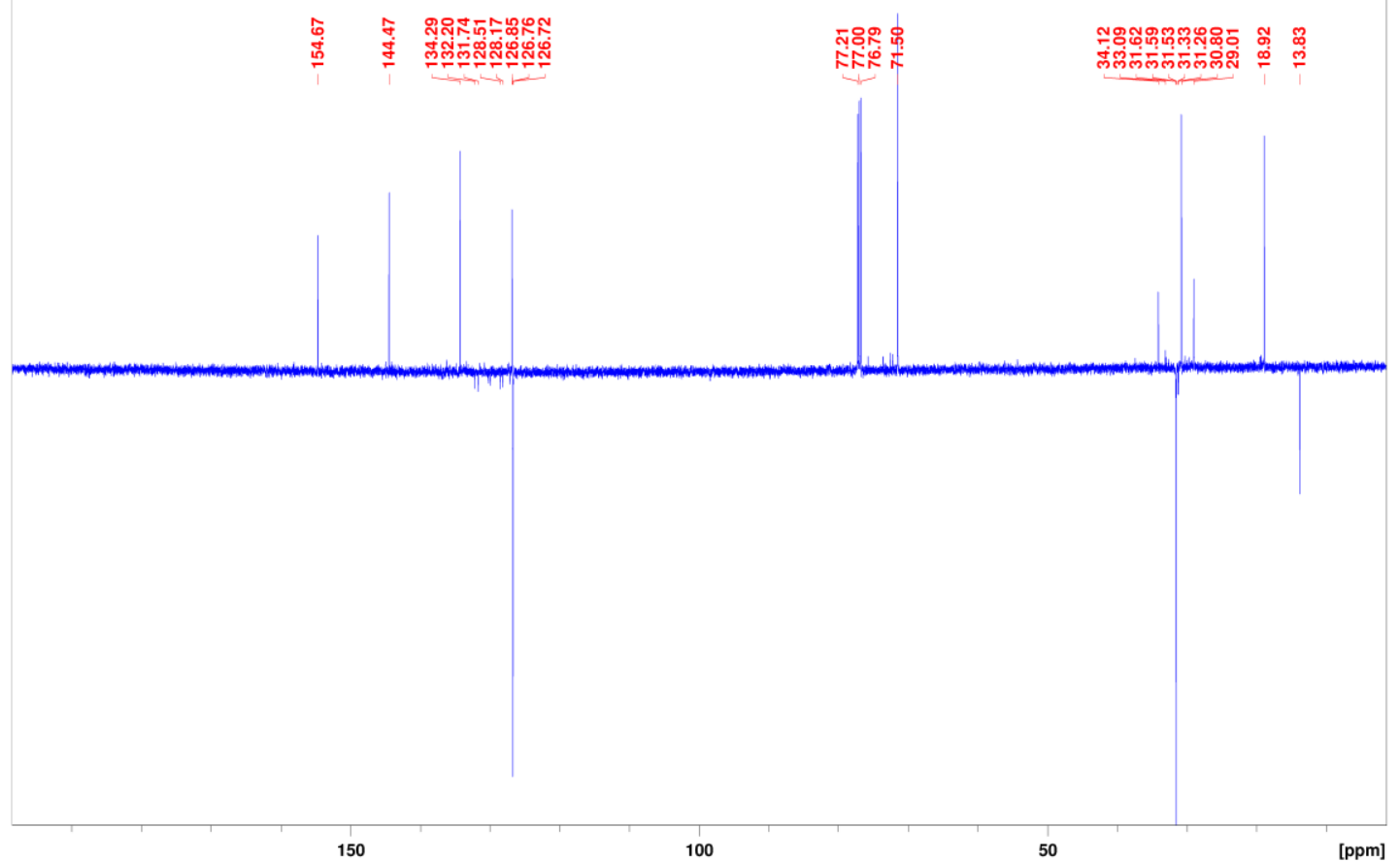

Figure S32: ${ }^{13} \mathrm{C}(\mathrm{APT}) \mathrm{NMR}$ of compound $4 \mathrm{Bu}\left(150.9 \mathrm{MHz}, \mathrm{CDCl}_{3}\right)$ 


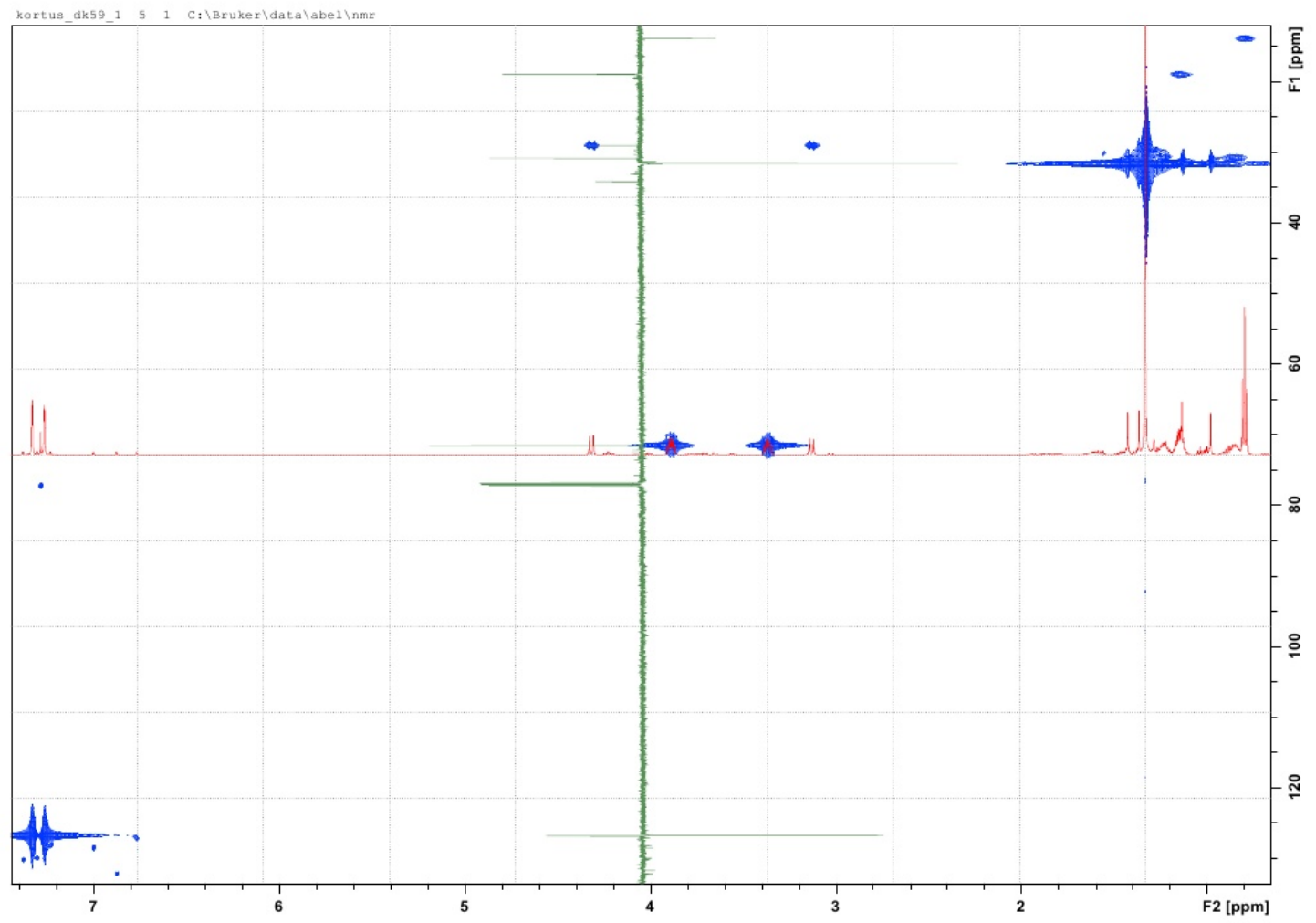

Figure S33: $\mathrm{HMQC} \mathrm{NMR} \mathrm{of} \mathrm{compound} \mathrm{4Bu}\left(600.1 \mathrm{MHz}, \mathrm{CDCl}_{3}\right)$

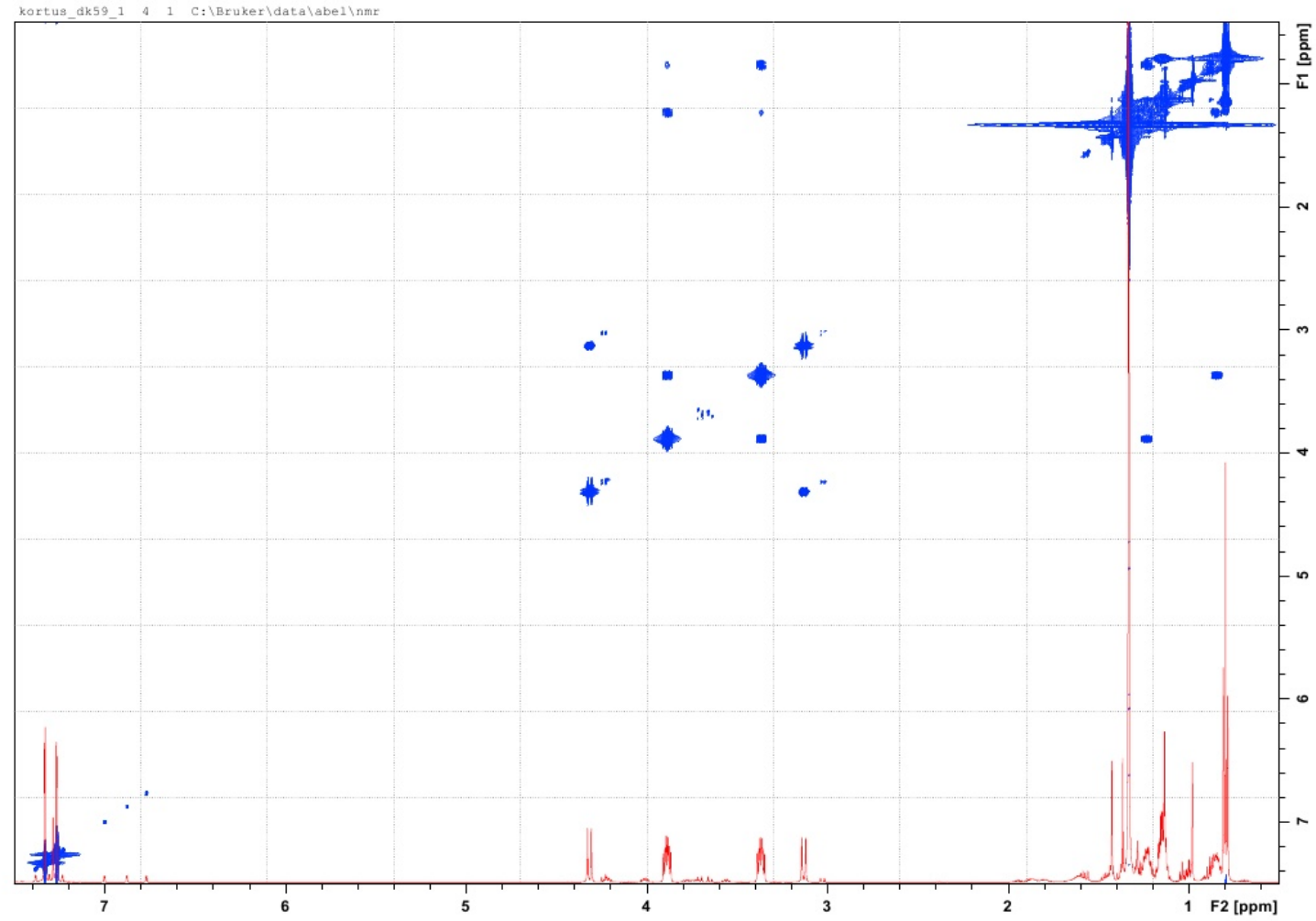

Figure S34: ${ }^{1} \mathrm{H}-{ }^{1} \mathrm{H}$ COSY NMR of compound 4Bu (600.1 MHz, $\mathrm{CDCl}_{3}$ ) 


\subsection{Compounds 5Et and 6Et}

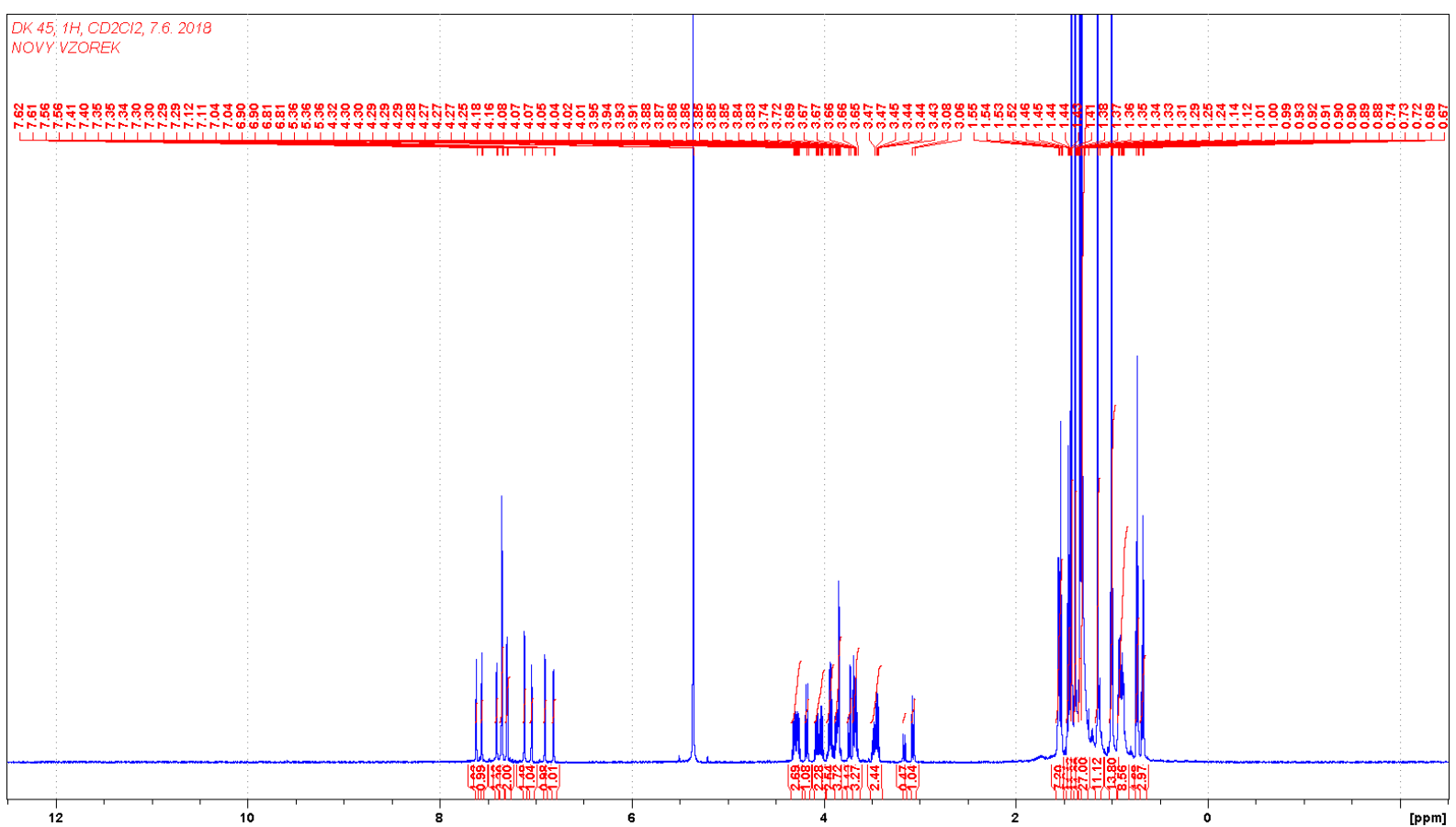

Figure S35: ${ }^{1} \mathrm{H}$ NMR of compounds $5 \mathrm{Et}$ and $6 \mathrm{Et}\left(600.1 \mathrm{MHz}, \mathrm{CD}_{2} \mathrm{Cl}_{2}\right)$

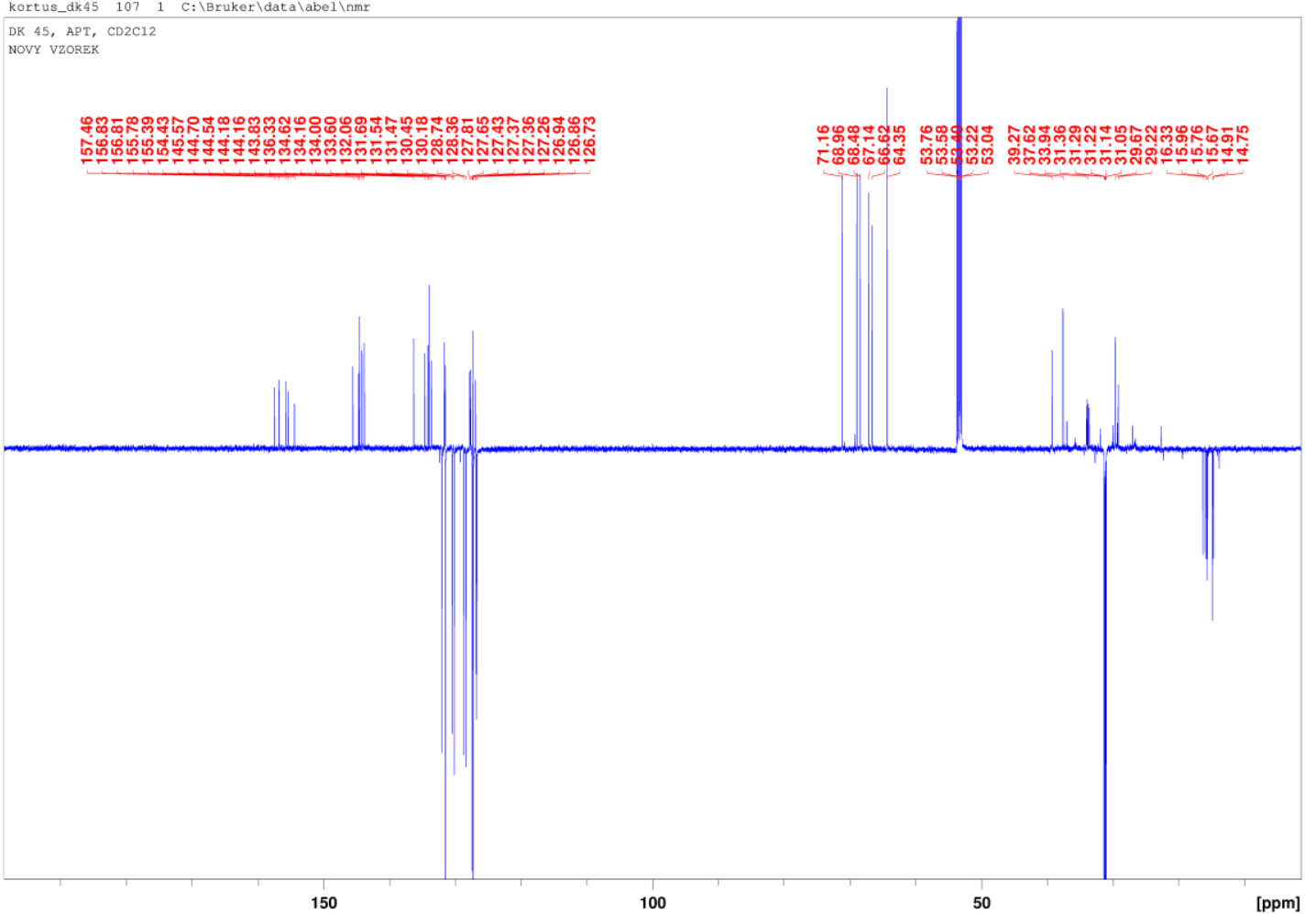

Figure S36: ${ }^{13} \mathrm{C}$ (APT) NMR of compounds $5 \mathrm{Et}$ and $6 \mathrm{Et}\left(150.9 \mathrm{MHz}, \mathrm{C}_{2} \mathrm{D}_{2} \mathrm{Cl}_{4}\right)$ 


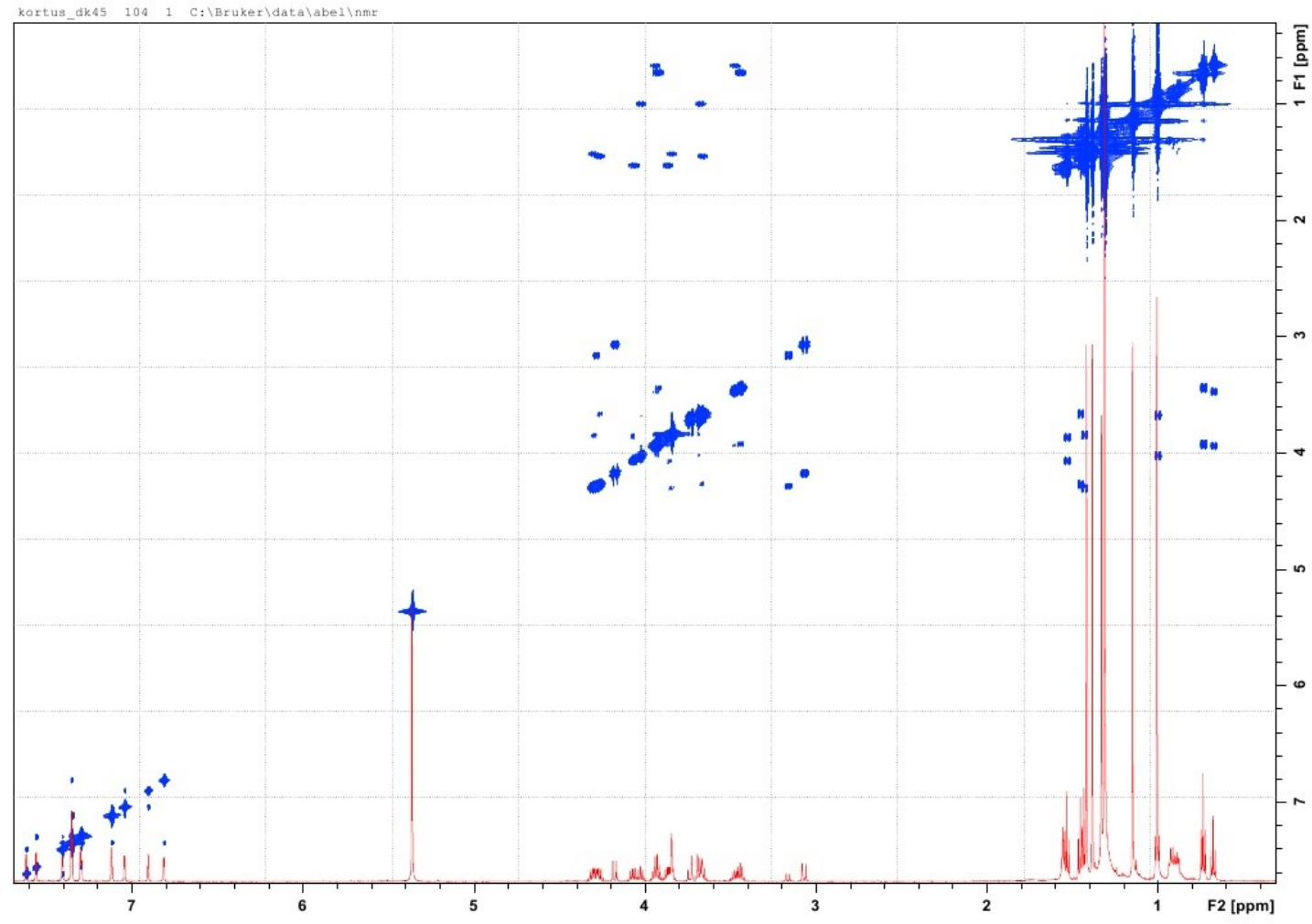

Figure S37: ${ }^{1} \mathrm{H}-{ }^{1} \mathrm{H}$ COSY NMR of compounds 5Et and 6Et $\left(600.1 \mathrm{MHz}, \mathrm{CD}_{2} \mathrm{Cl}_{2}\right)$

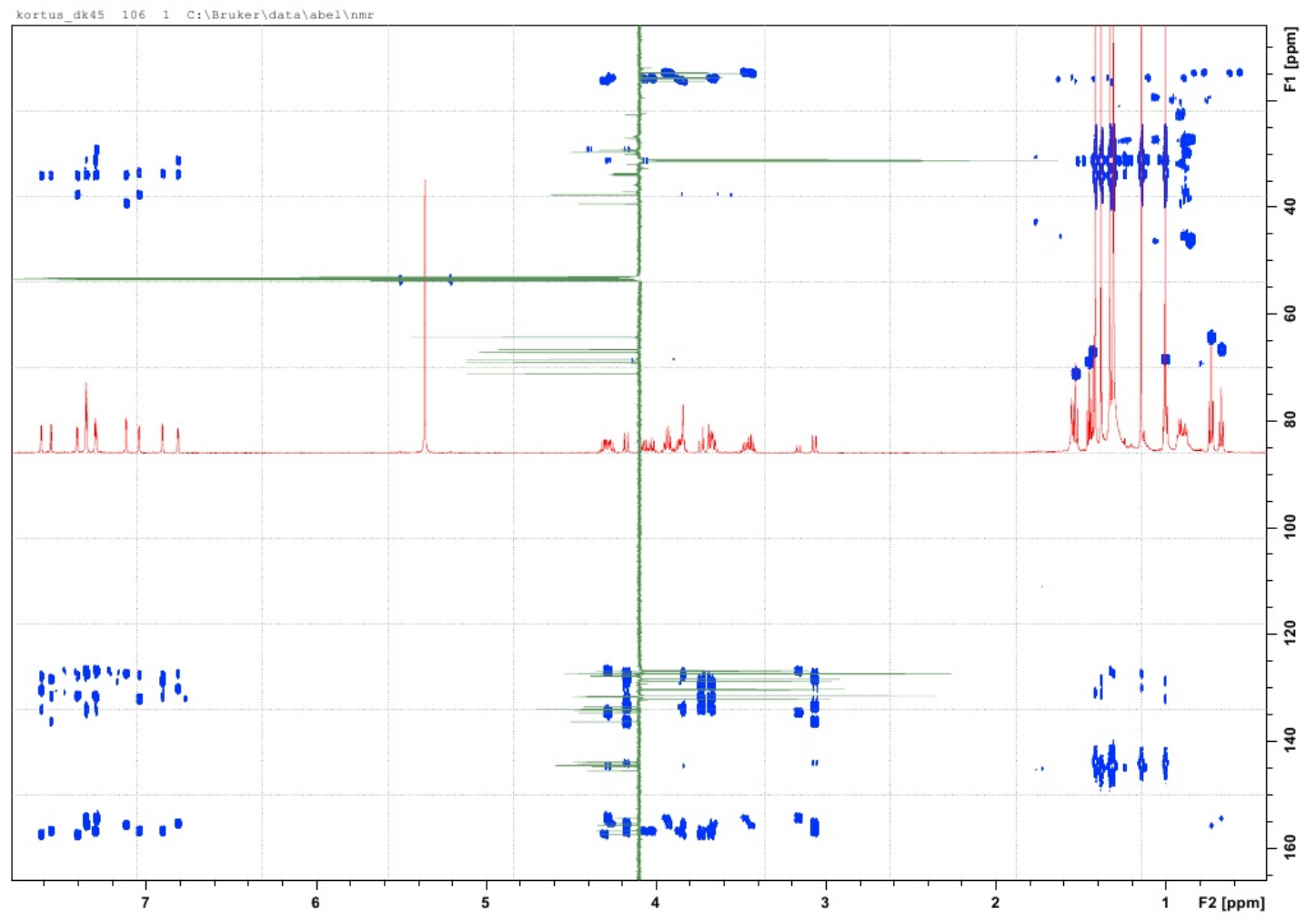

Figure S38: $\mathrm{HMBC}$ NMR of compounds $5 \mathrm{Et}$ and $6 \mathrm{Et}\left(600.1 \mathrm{MHz}, \mathrm{CD}_{2} \mathrm{Cl}_{2}\right)$ 


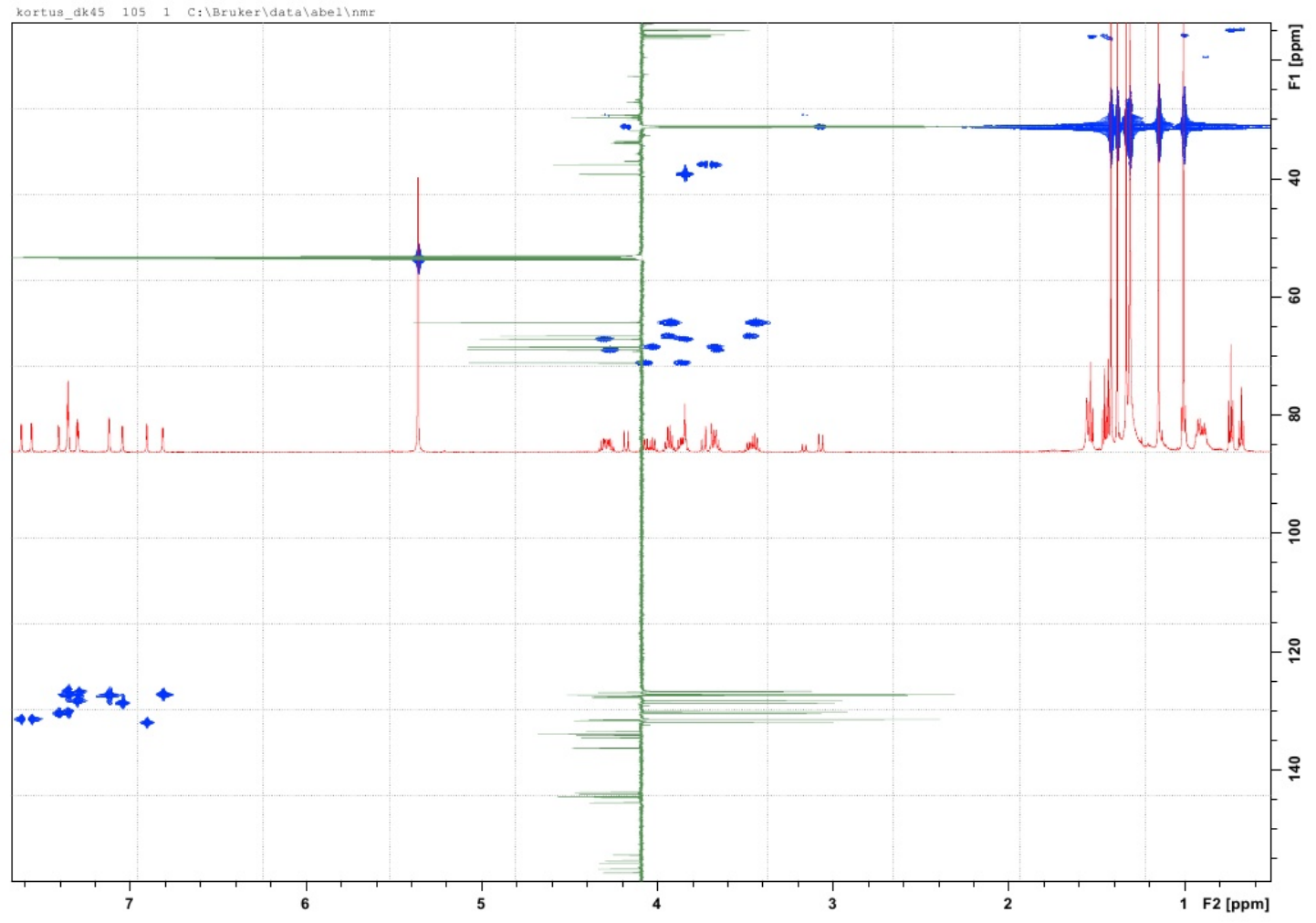

Figure S39: HMQC NMR of compounds 5Et and 6Et $\left(600.1 \mathrm{MHz}, \mathrm{CD}_{2} \mathrm{Cl}_{2}\right)$ 


\subsection{Compound 5Pr}

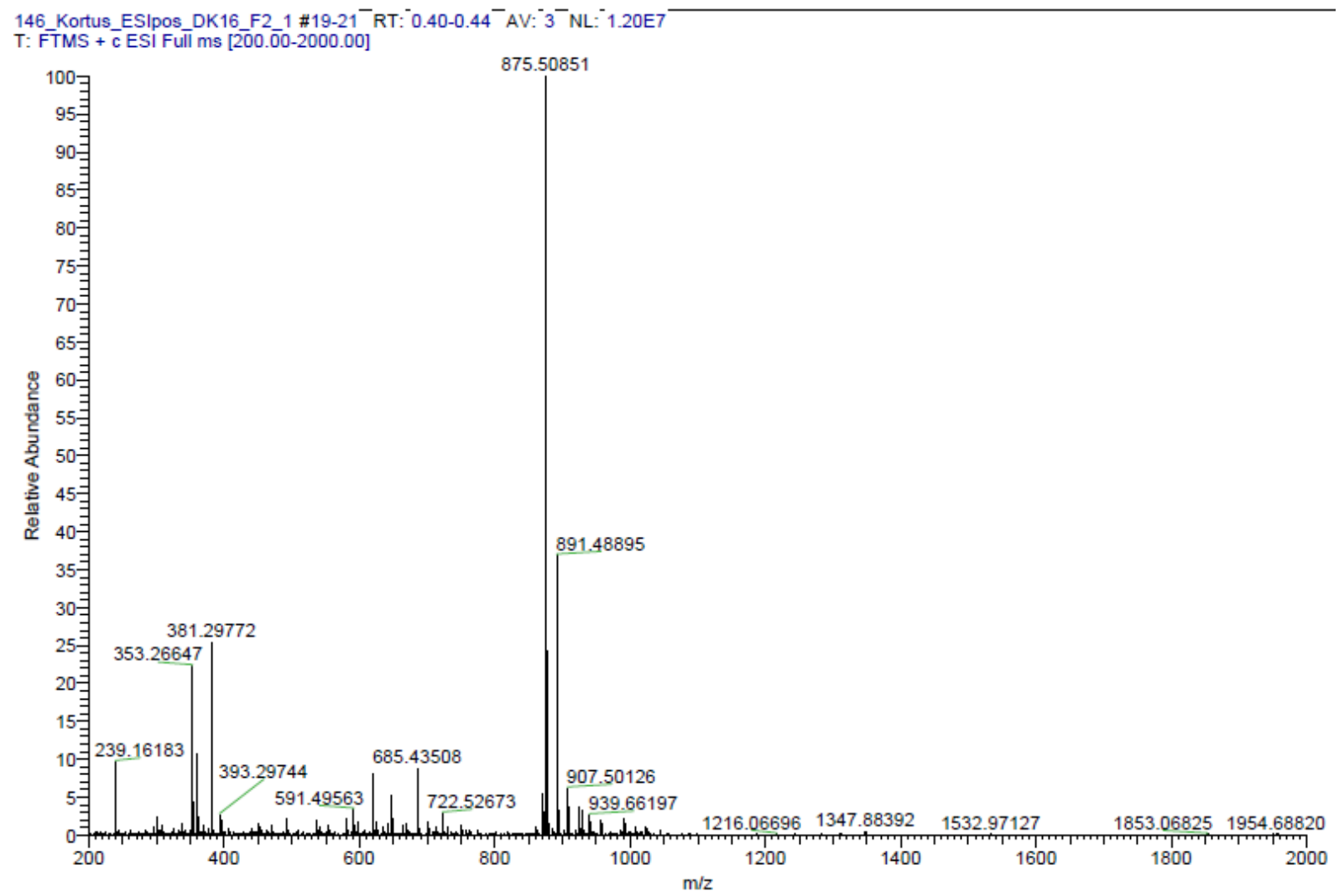

Figure S40: HRMS of compound $5 \mathrm{Pr}\left(\mathrm{ESI}^{+}\right)$

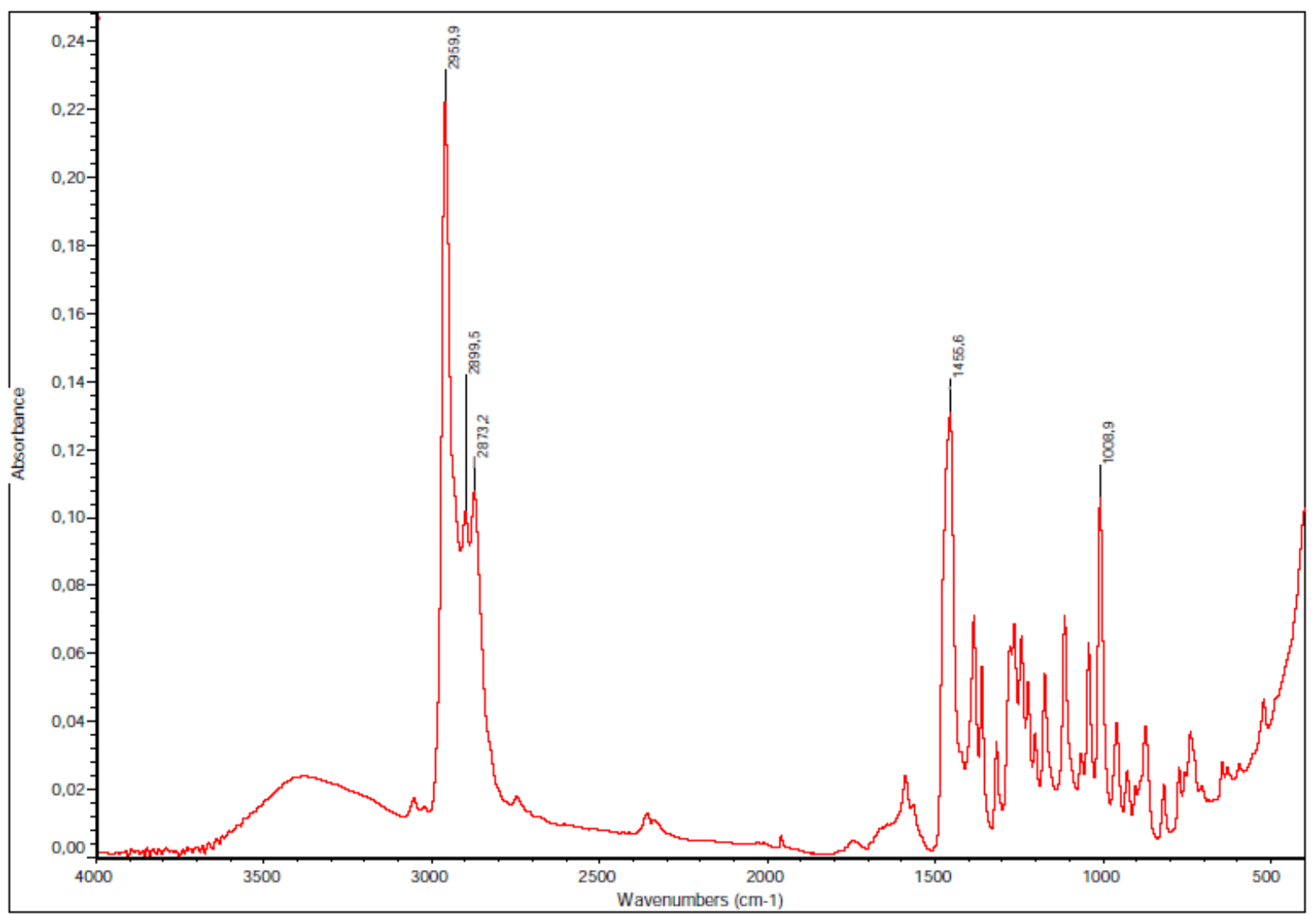

Figure S41: IR of compound $5 \operatorname{Pr}(\mathrm{KBr})$ 


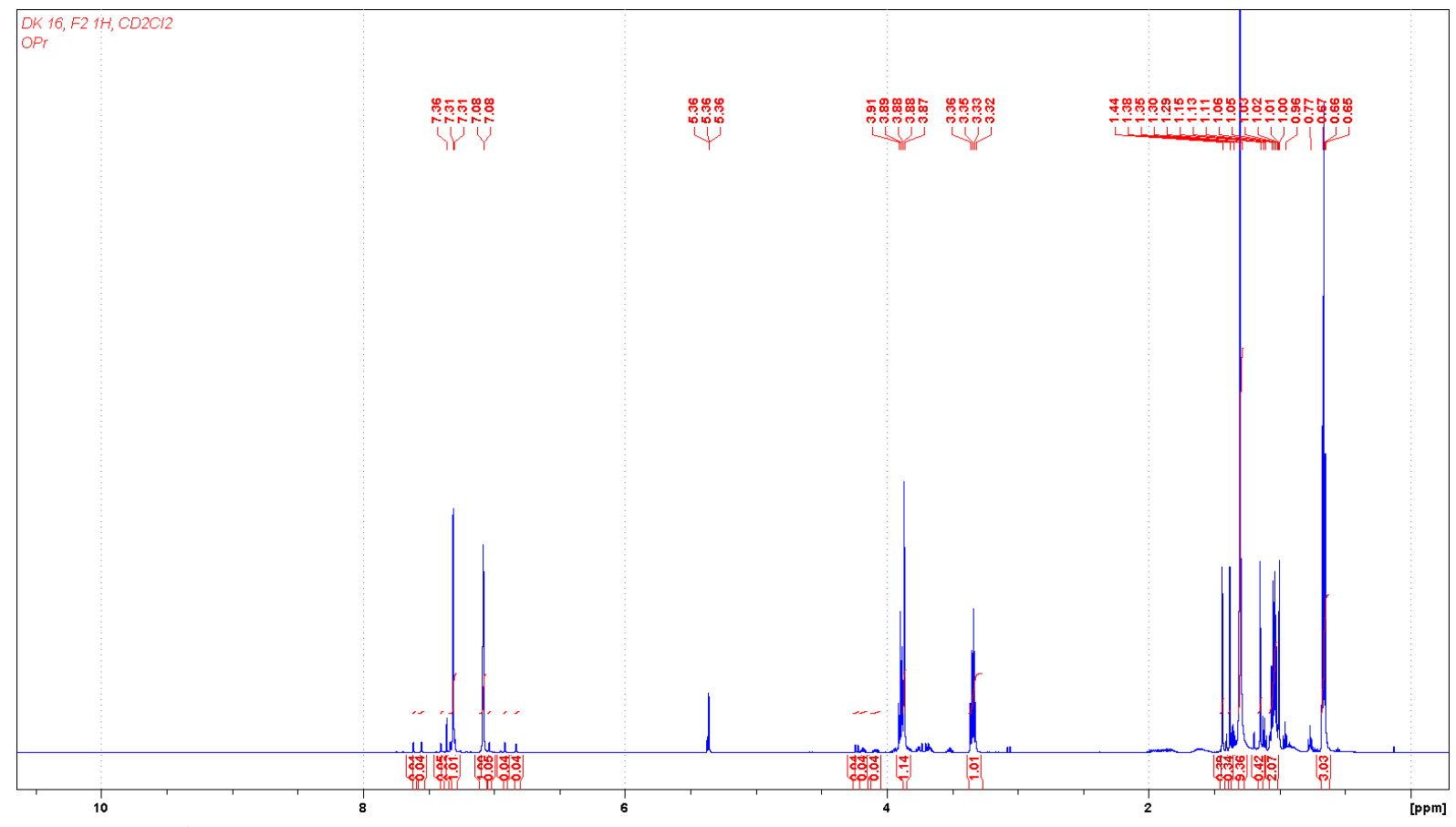

Figure S42: ${ }^{1} \mathrm{H}$ NMR of compound $5 \operatorname{Pr}\left(600.1 \mathrm{MHz}, \mathrm{CD}_{2} \mathrm{Cl}_{2}\right)$

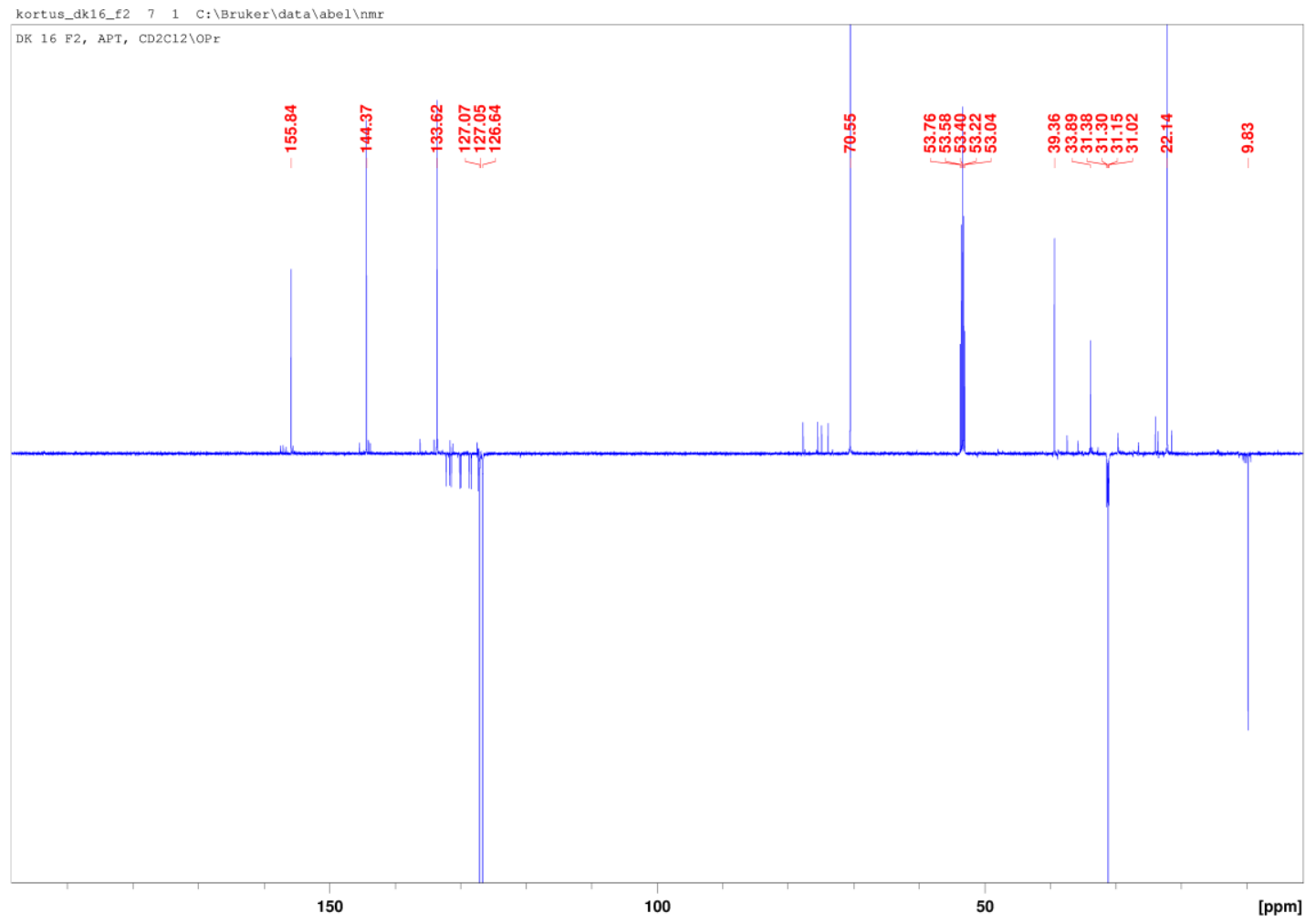

Figure S43: ${ }^{13} \mathrm{C}(\mathrm{APT}) \mathrm{NMR}$ of compound $\operatorname{PPr}\left(150.9 \mathrm{MHz}, \mathrm{CD}_{2} \mathrm{Cl}_{2}\right)$ 


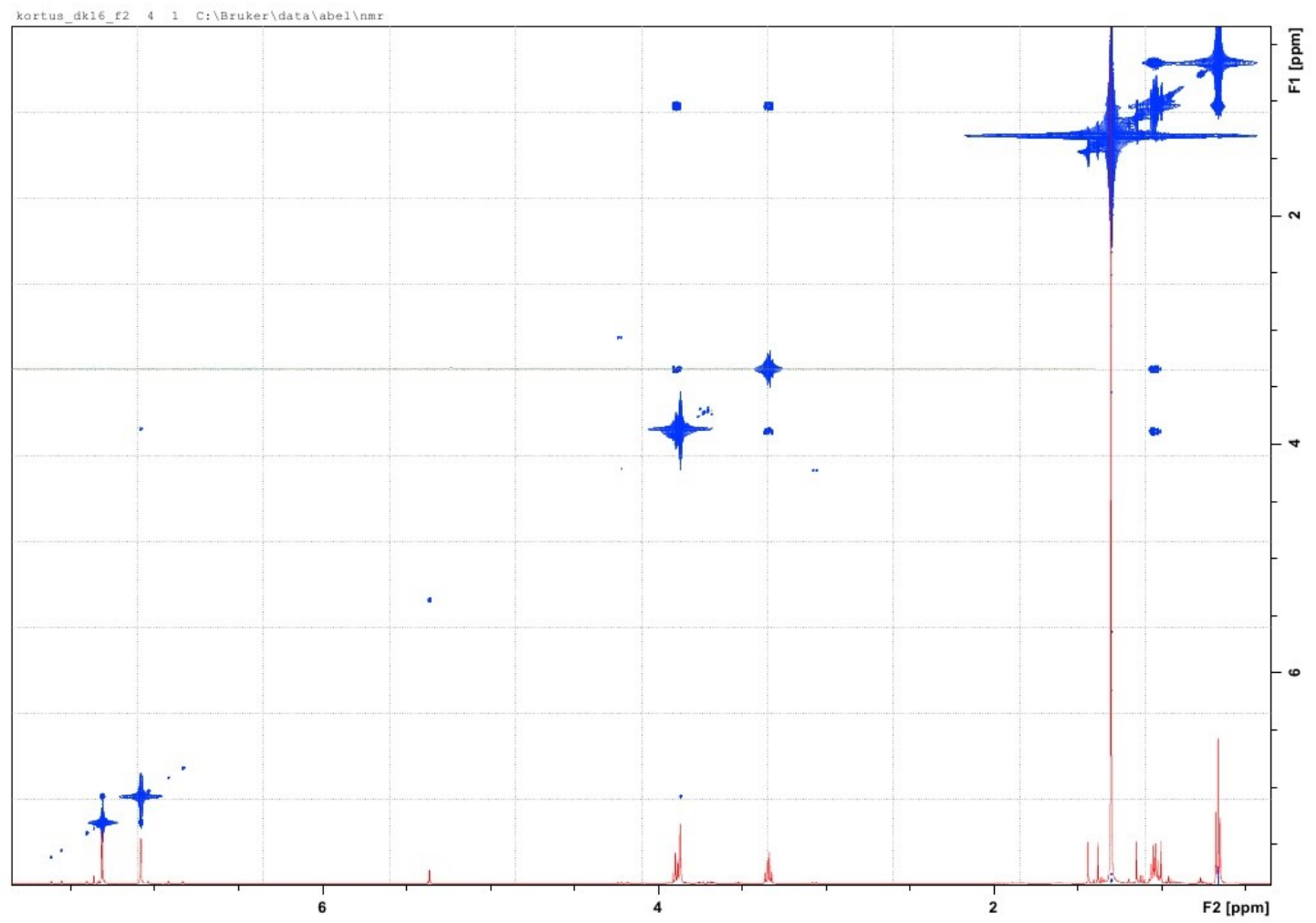

Figure S44: ${ }^{1} \mathrm{H}-{ }^{1} \mathrm{H}$ COSY NMR of compound $5 \operatorname{Pr}\left(600.1 \mathrm{MHz}, \mathrm{CD}_{2} \mathrm{Cl}_{2}\right)$

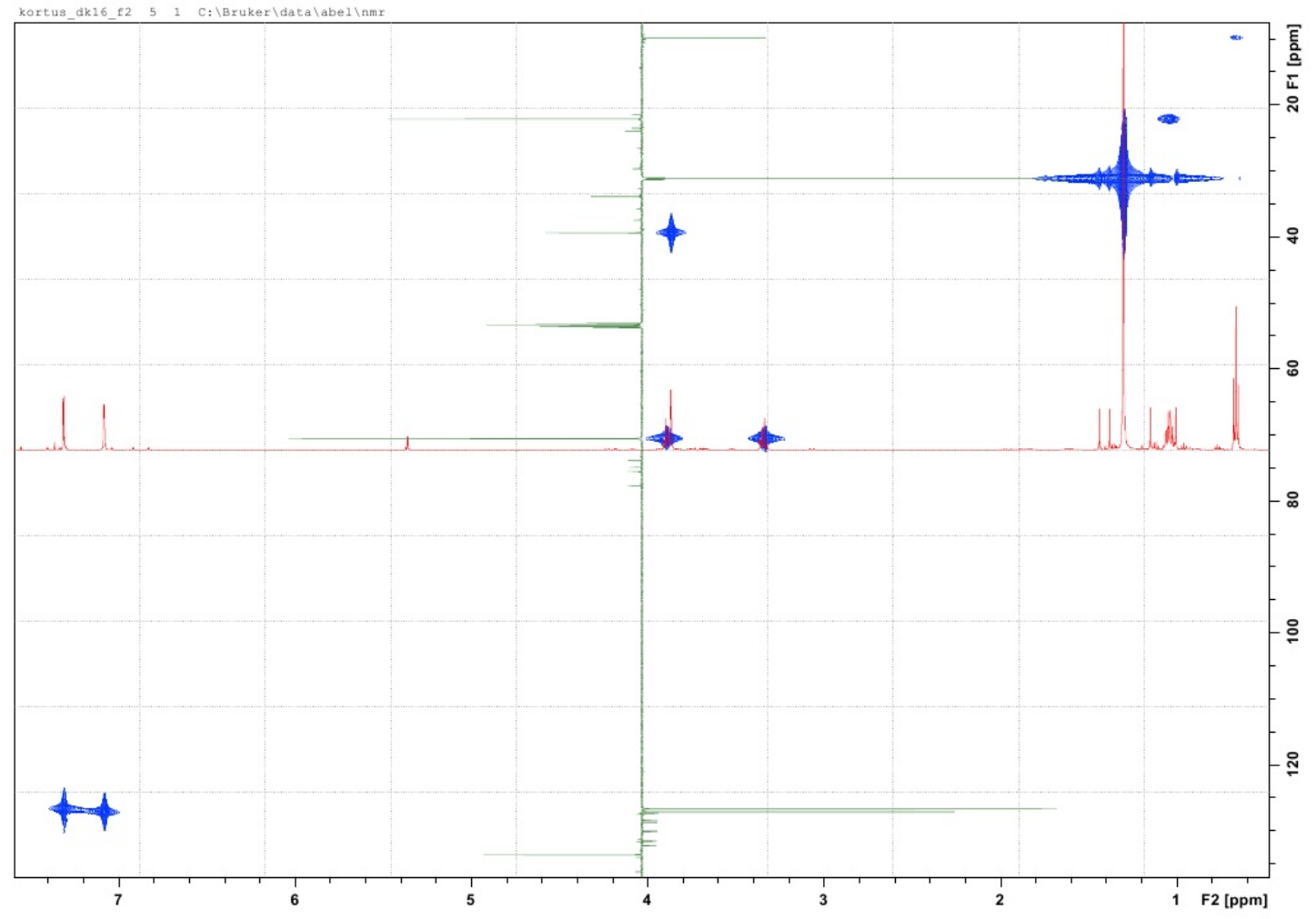

Figure S45: $\mathrm{HMQC} \mathrm{NMR}$ of compound $5 \operatorname{Pr}\left(600.1 \mathrm{MHz}, \mathrm{CD}_{2} \mathrm{Cl}_{2}\right)$ 


\subsection{Compound 5Bu}

316_Kortus_ESIpos_DK59_3_1 \#47-52 RT: 0.68-0.75 AV: 6 NL: $1.02 E 7$

T: FTMS + c ESI Full ms [200.00-2000.00]

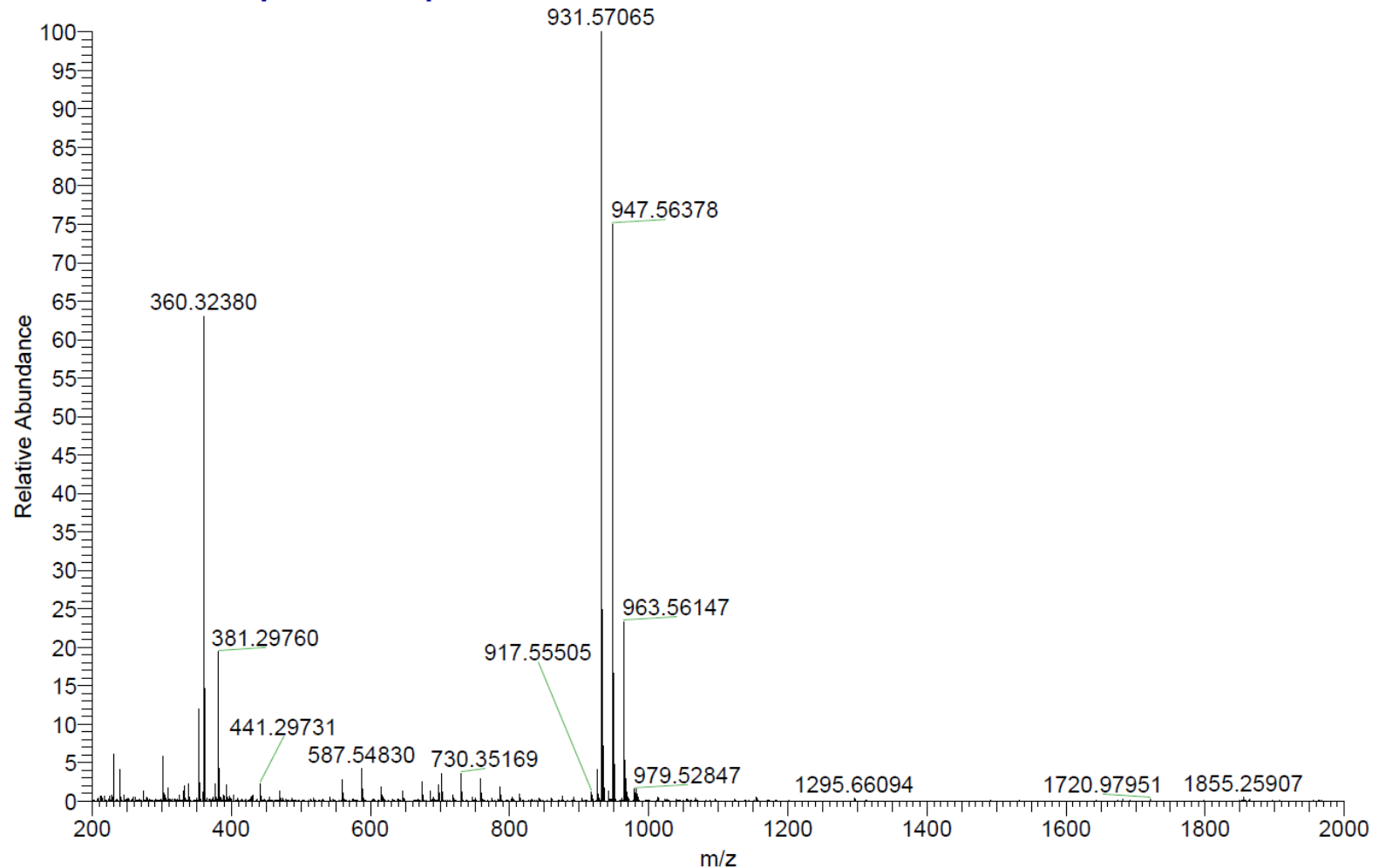

Figure S46: HRMS of compound $5 \mathrm{Bu}\left(\mathrm{ESI}^{+}\right)$

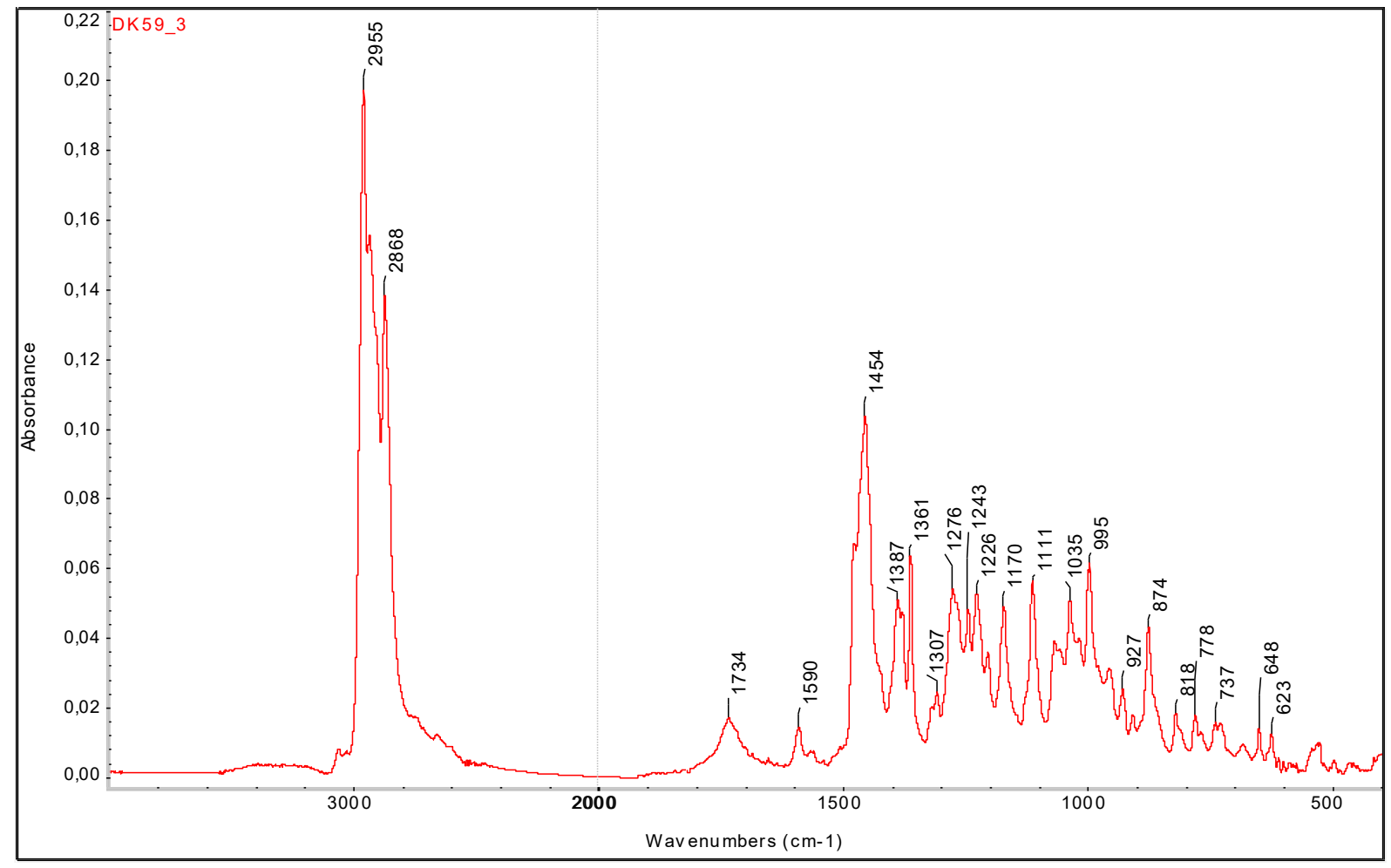

Figure S47: IR of compound $5 \mathrm{Bu}(\mathrm{KBr})$ 


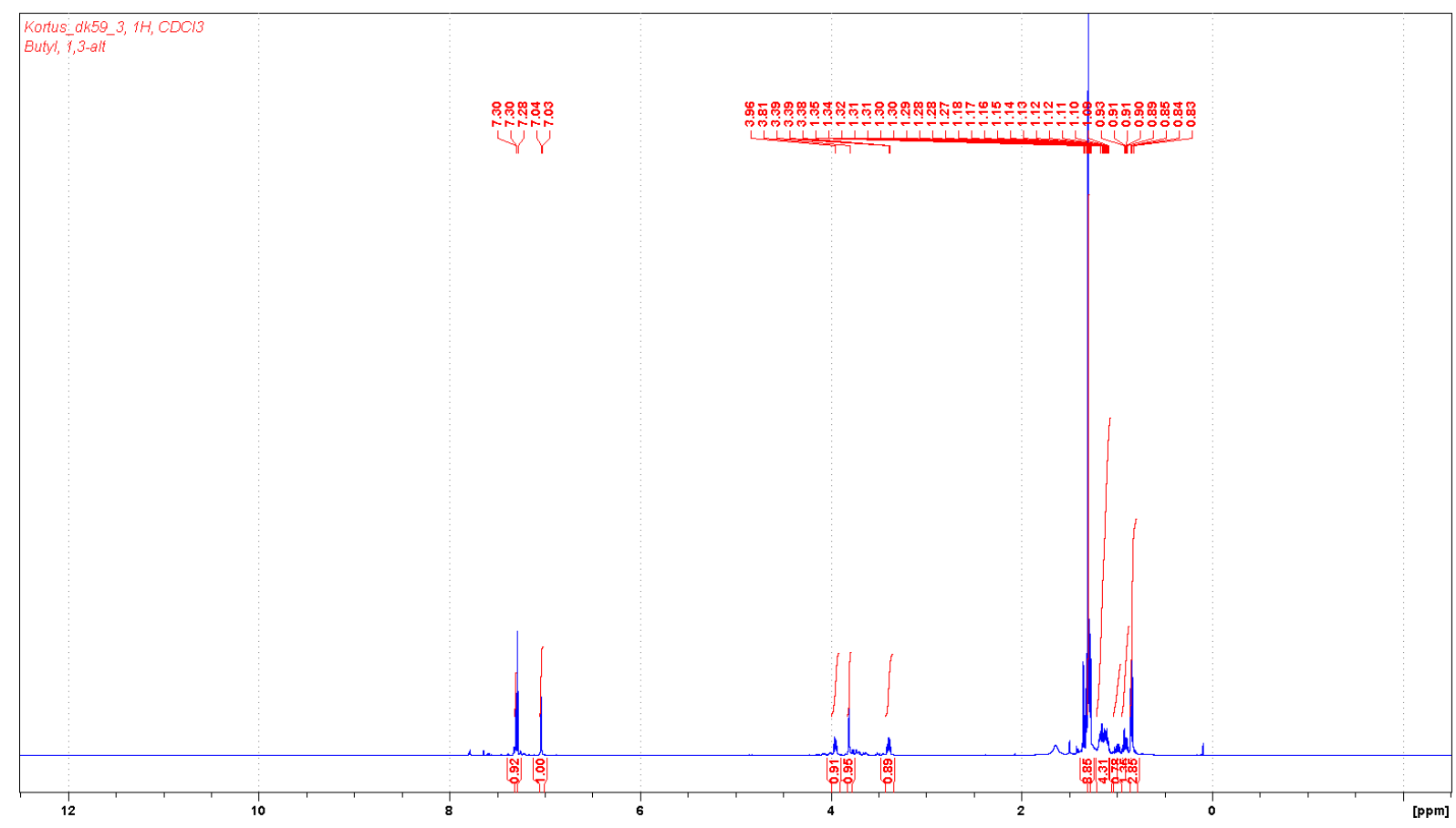

Figure S48: ${ }^{1} \mathrm{H}$ NMR of compound $5 \mathrm{Bu}\left(600.1 \mathrm{MHz}, \mathrm{CDCl}_{3}\right)$

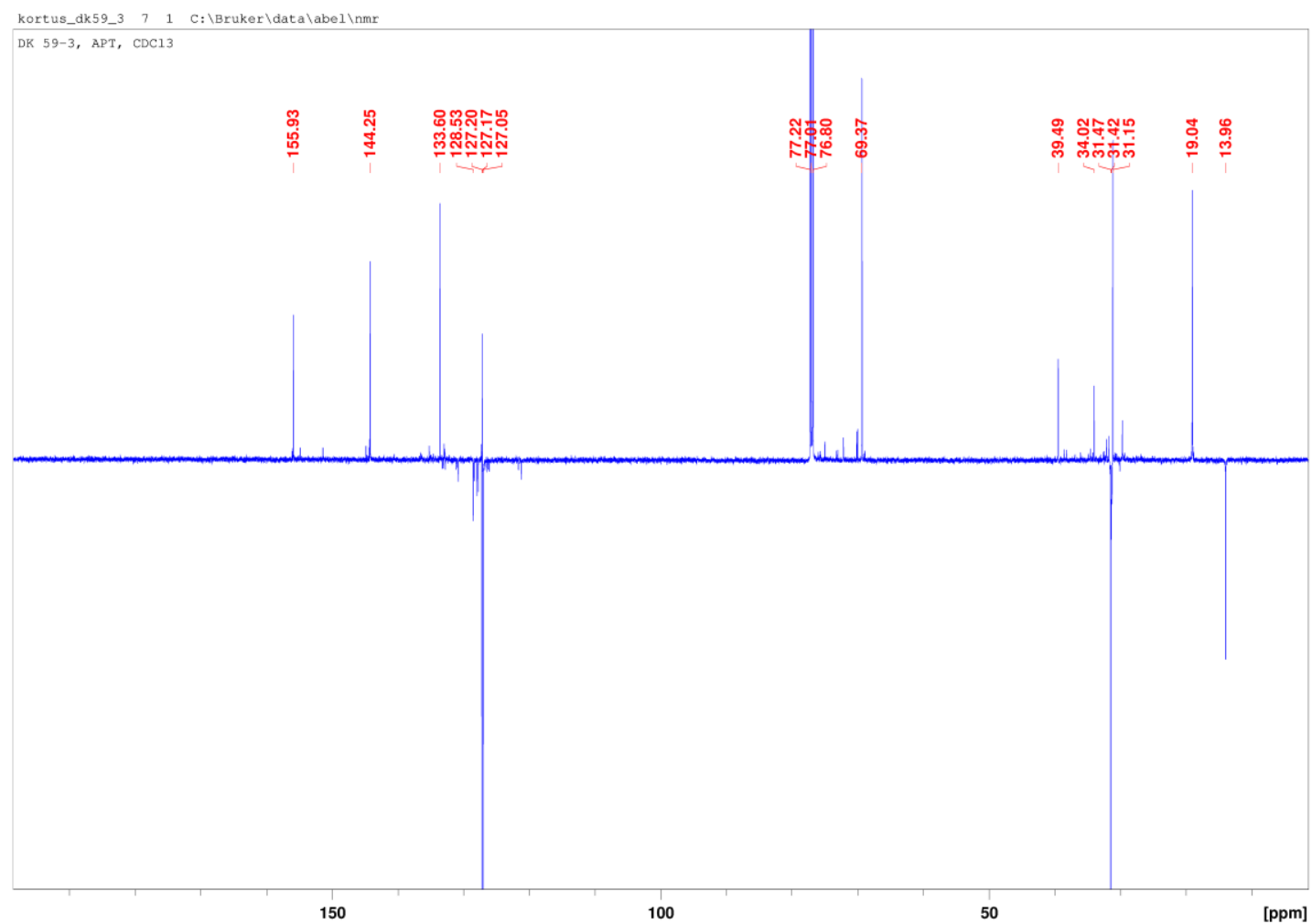

Figure S49: ${ }^{13} \mathrm{C}$ (APT) NMR of compound $5 \mathrm{Bu}\left(150.9 \mathrm{MHz}, \mathrm{CDCl}_{3}\right)$ 


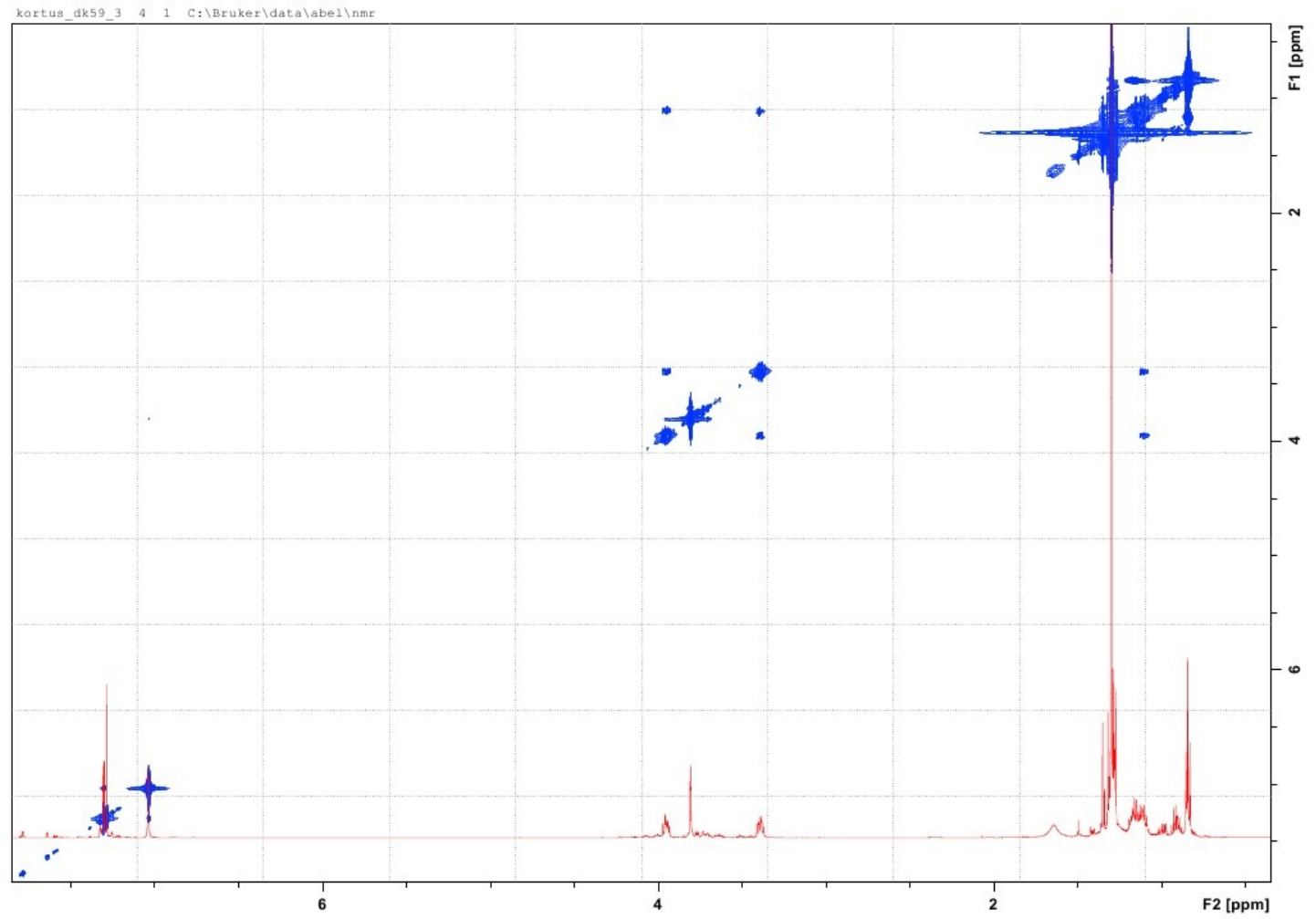

Figure S50: ${ }^{1} \mathrm{H}-{ }^{1} \mathrm{H}$ COSY NMR of compound $5 \mathrm{Bu}\left(600.1 \mathrm{MHz}, \mathrm{CDCl}_{3}\right)$

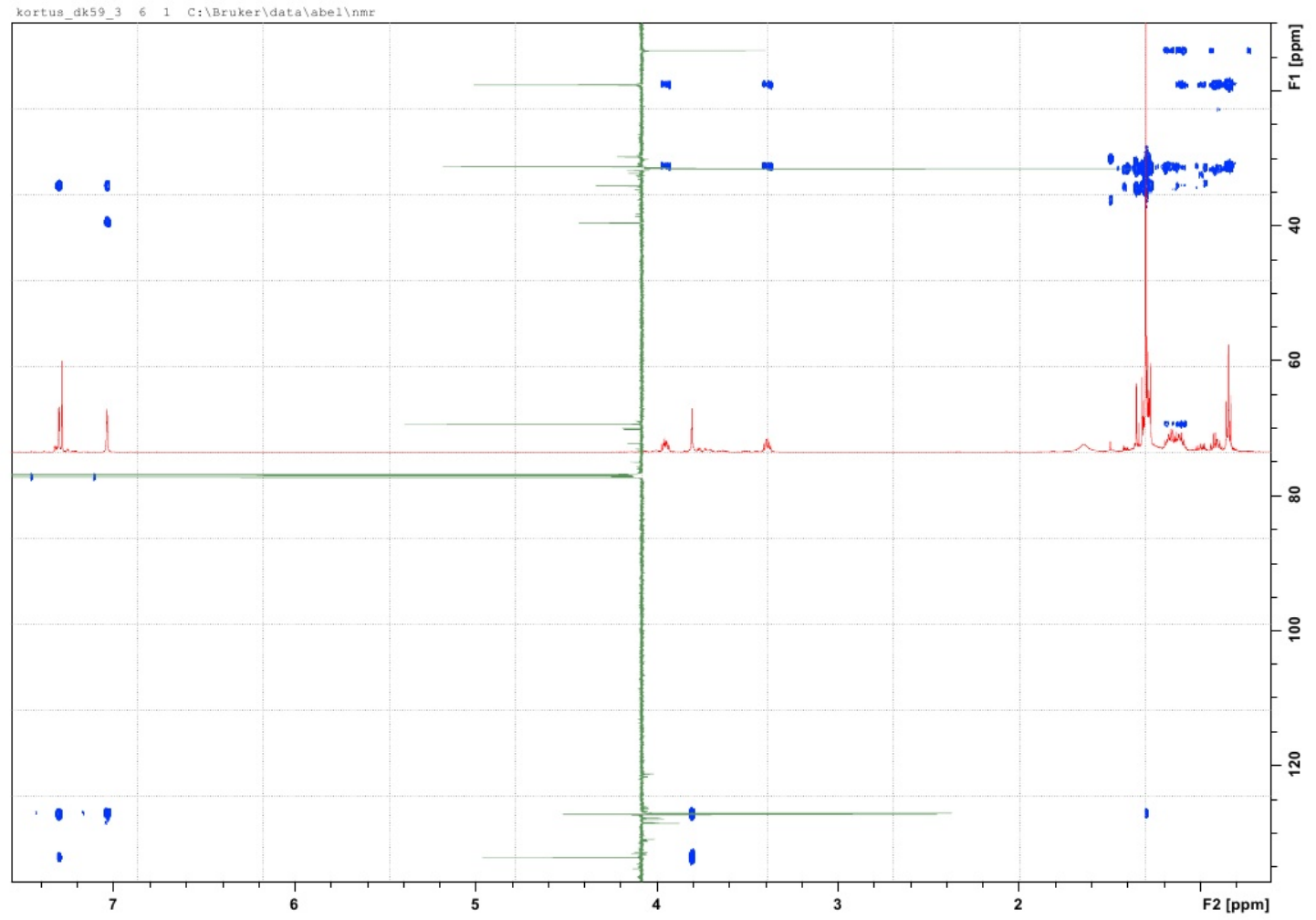

Figure S51: $\mathrm{HMBC}$ NMR of compound $5 \mathrm{Bu}\left(600.1 \mathrm{MHz}, \mathrm{CDCl}_{3}\right)$ 


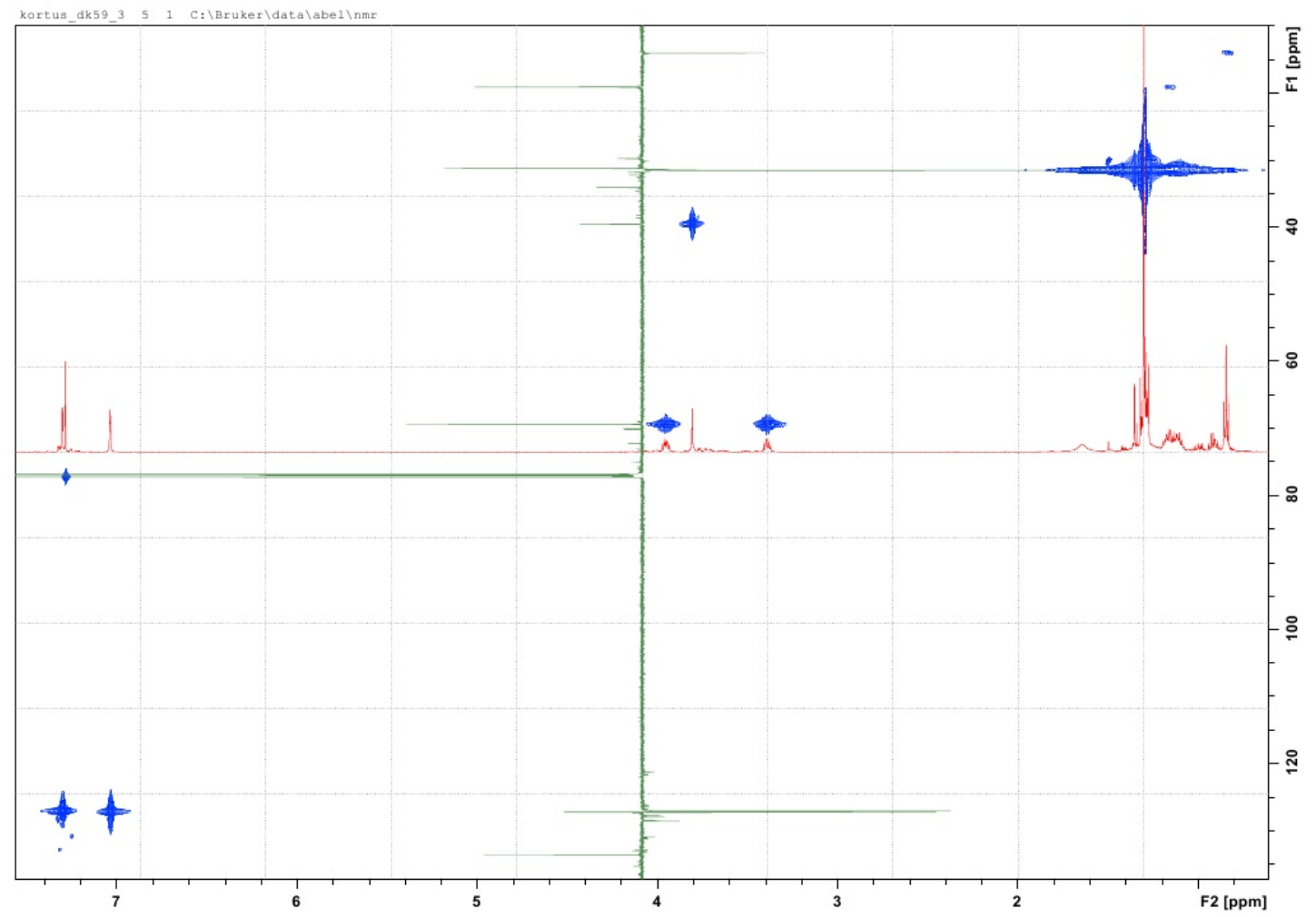

Figure S52: $\mathrm{HMQC} \mathrm{NMR} \mathrm{of} \mathrm{compound} \mathrm{5Bu}\left(600.1 \mathrm{MHz}, \mathrm{CDCl}_{3}\right)$ 


\subsection{Compound 6Pr}

330_Kortus_ESIpos_DK 56_P_1 \#52-56 RT: 0.75-0.81 AV: 5 NL: 1.02E7

T: FTMS + c ESI Full ms [200.00-2000.00]

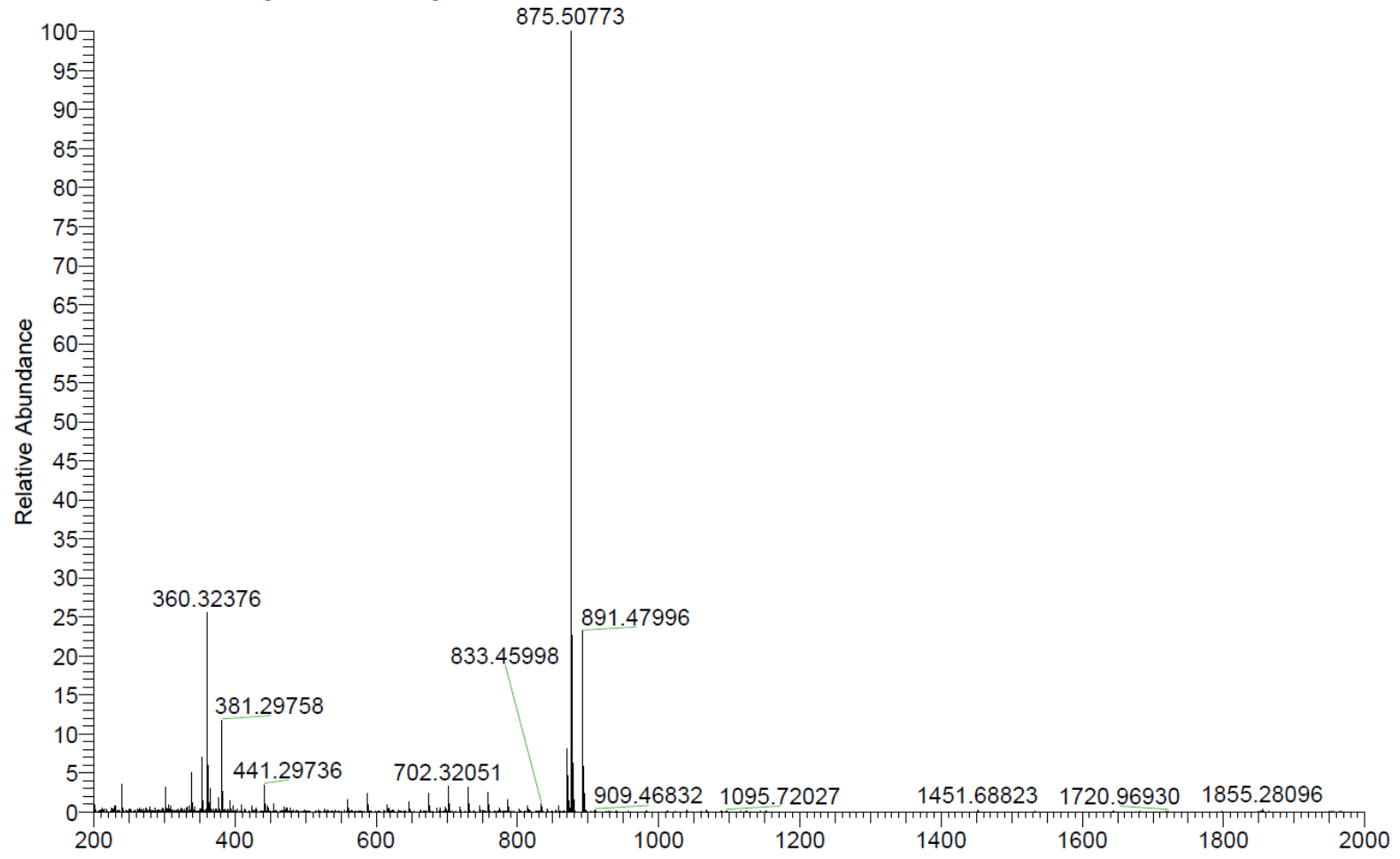

Figure S53: HRMS of compound $6 \operatorname{Pr}\left(\mathrm{ESI}^{+}\right)$

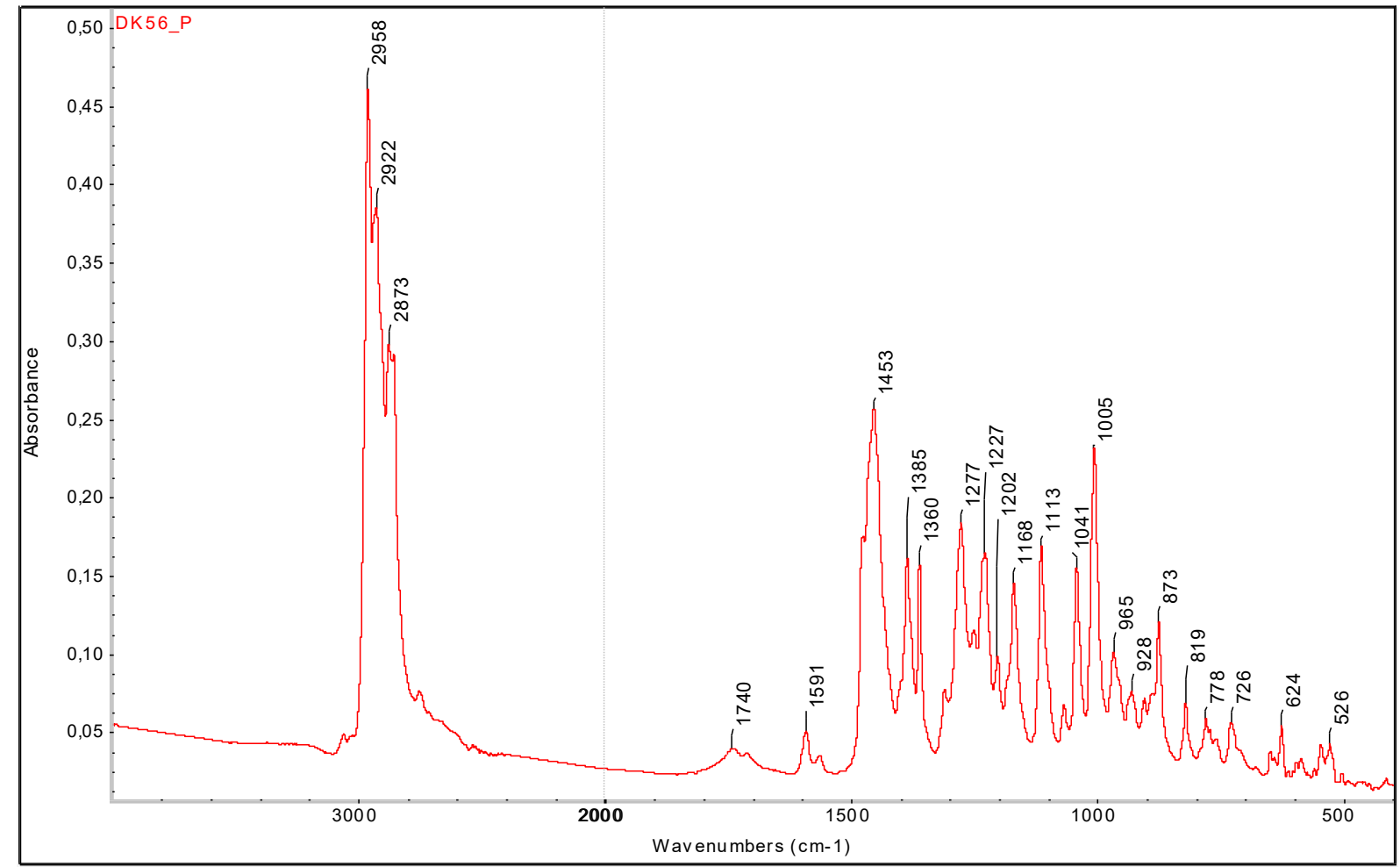

Figure S54: IR of compound 6Pr ( $\mathrm{KBr})$ 


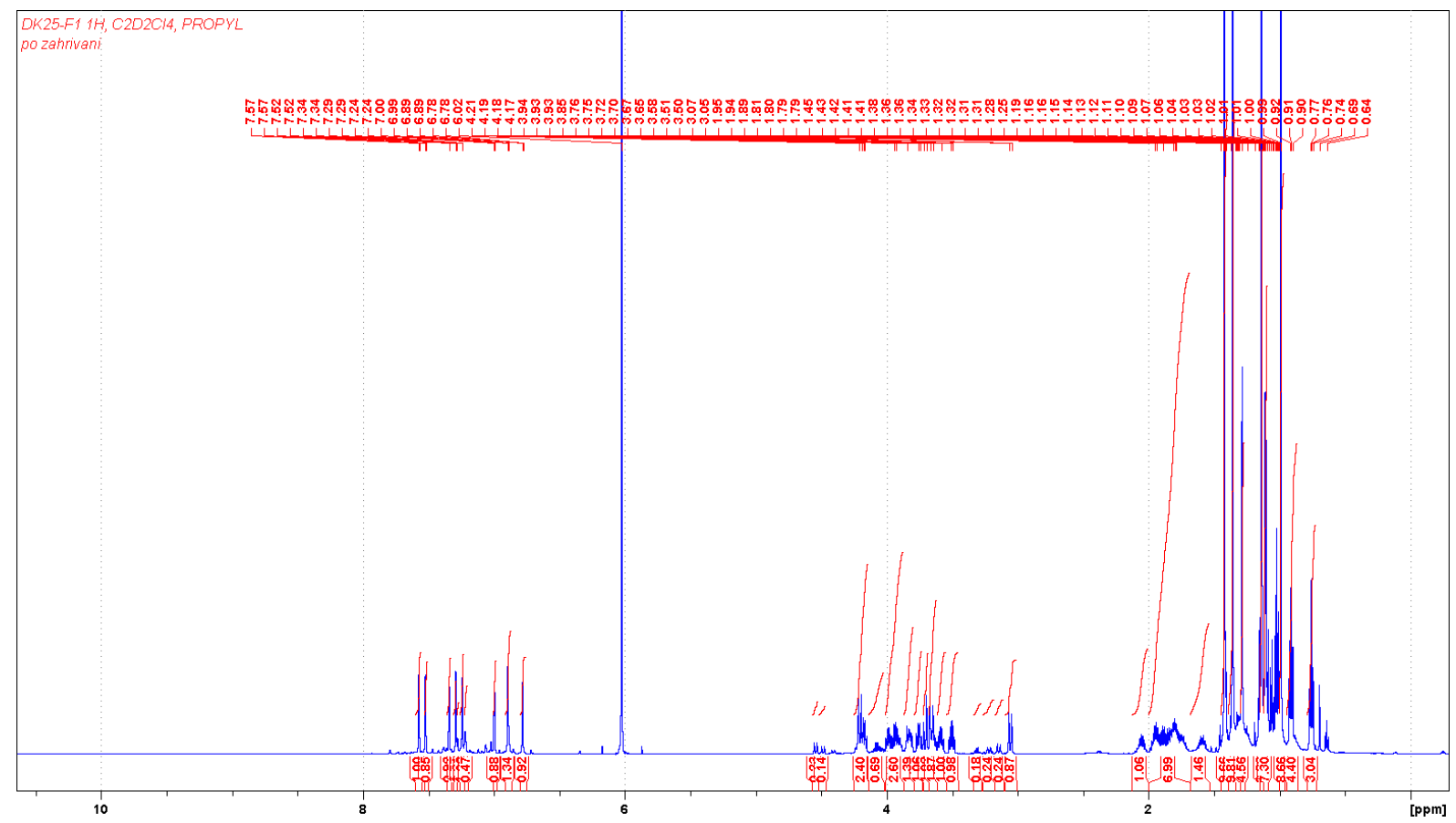

Figure S55: ${ }^{1} \mathrm{H}$ NMR of compound $6 \operatorname{Pr}\left(500.1 \mathrm{MHz}, \mathrm{C}_{2} \mathrm{D}_{2} \mathrm{Cl}_{4}\right)$

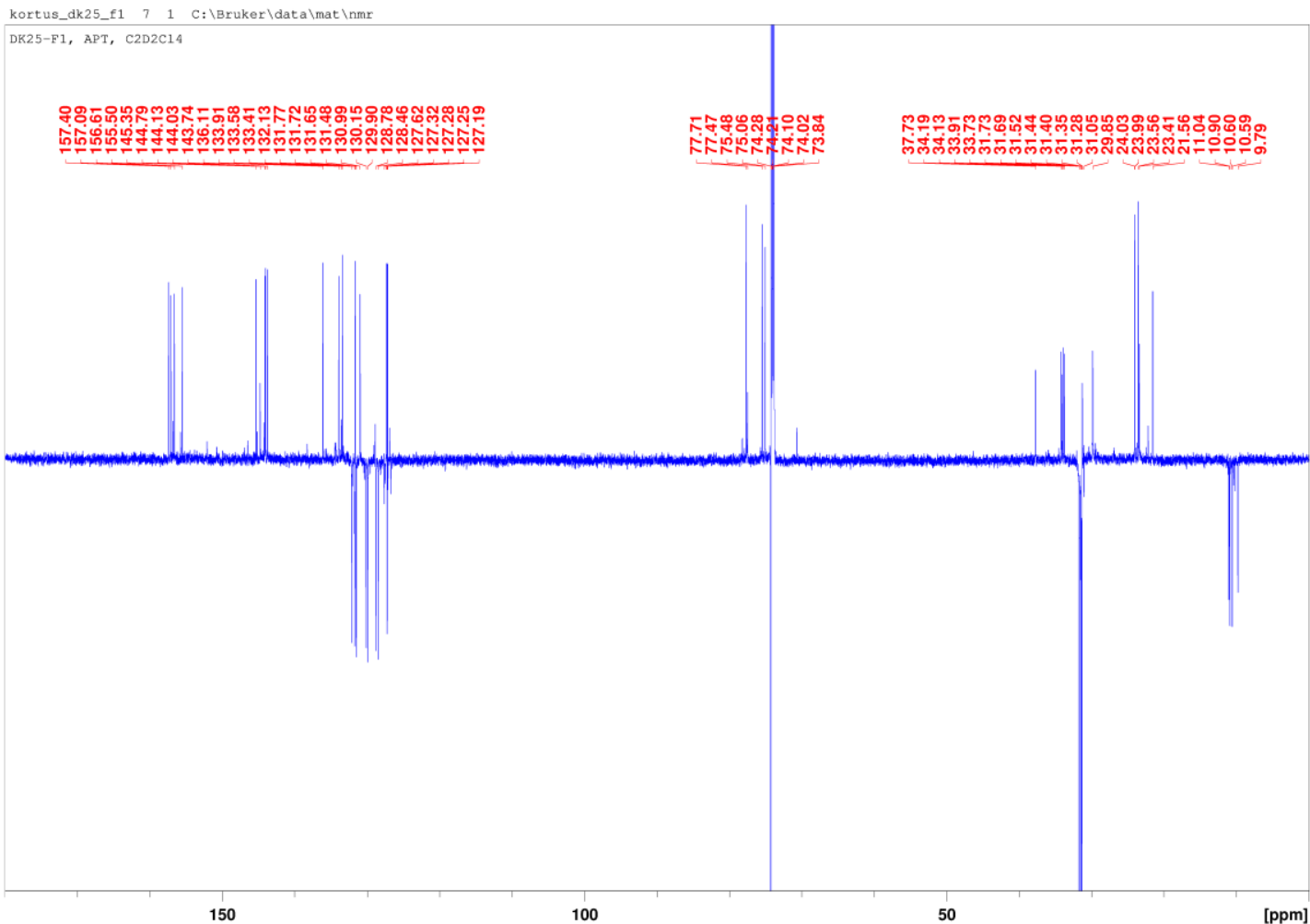

Figure S56: ${ }^{13} \mathrm{C}(\mathrm{APT}) \mathrm{NMR}$ of compound $6 \operatorname{Pr}\left(125.8 \mathrm{MHz}, \mathrm{C}_{2} \mathrm{D}_{2} \mathrm{Cl}_{4}\right)$ 


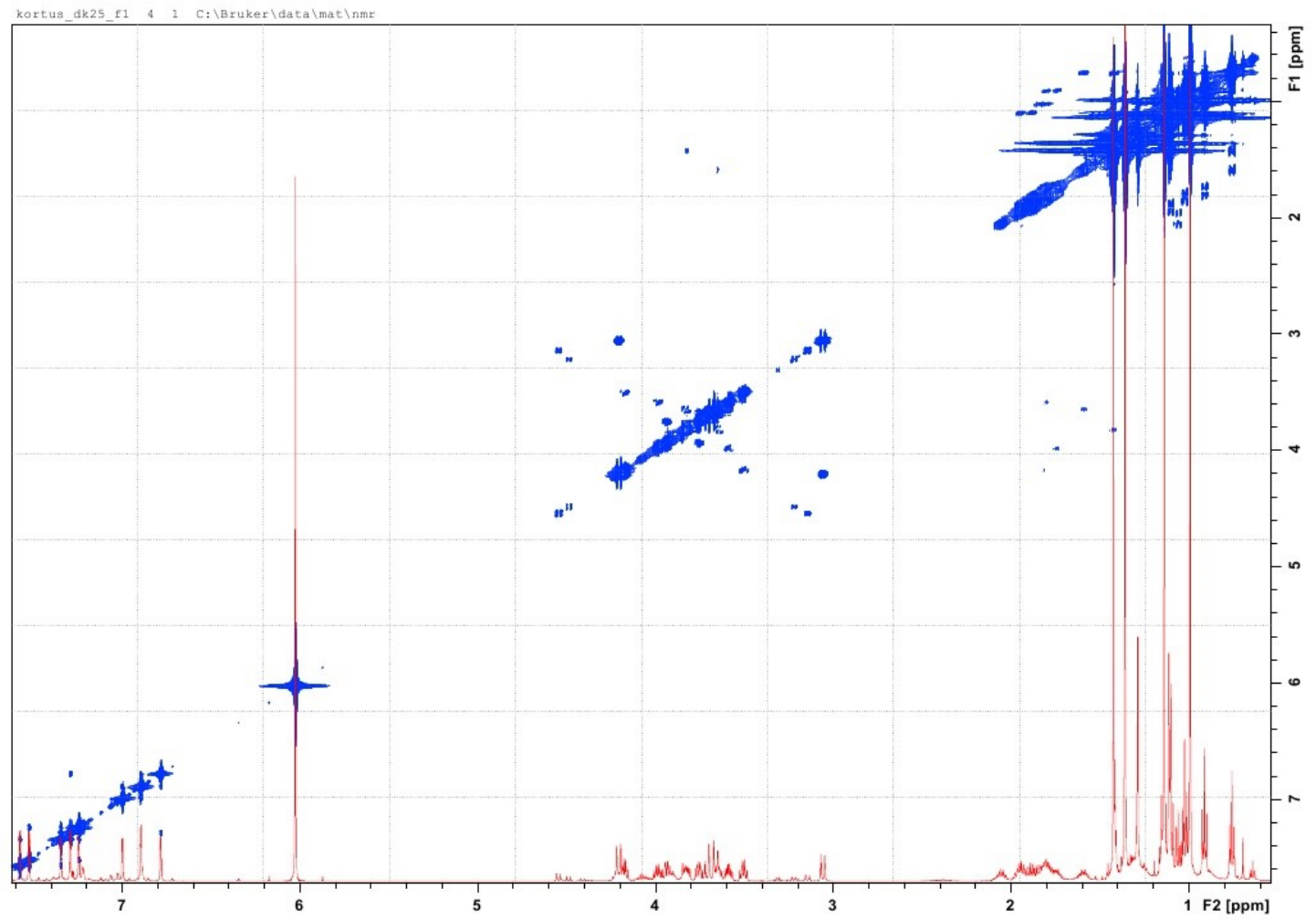

Figure S57: ${ }^{1} \mathrm{H}-{ }^{1} \mathrm{H}$ COSY NMR of compound $6 \operatorname{Pr}\left(500.1 \mathrm{MHz}, \mathrm{C}_{2} \mathrm{D}_{2} \mathrm{Cl}_{4}\right)$

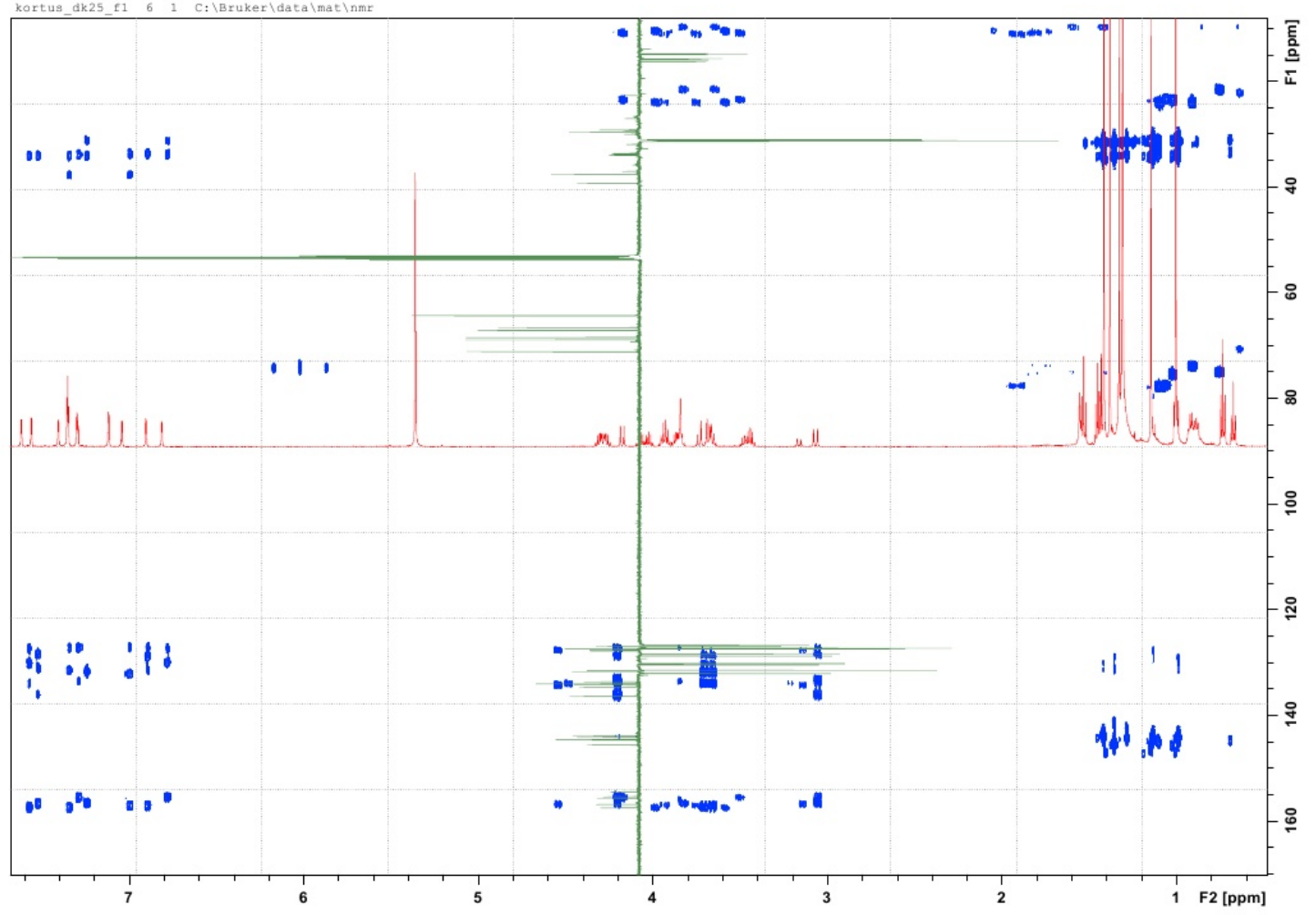

Figure S58: $\mathrm{HMBC}$ NMR of compound $6 \operatorname{Pr}\left(500.1 \mathrm{MHz}, \mathrm{C}_{2} \mathrm{D}_{2} \mathrm{Cl}_{4}\right)$ 


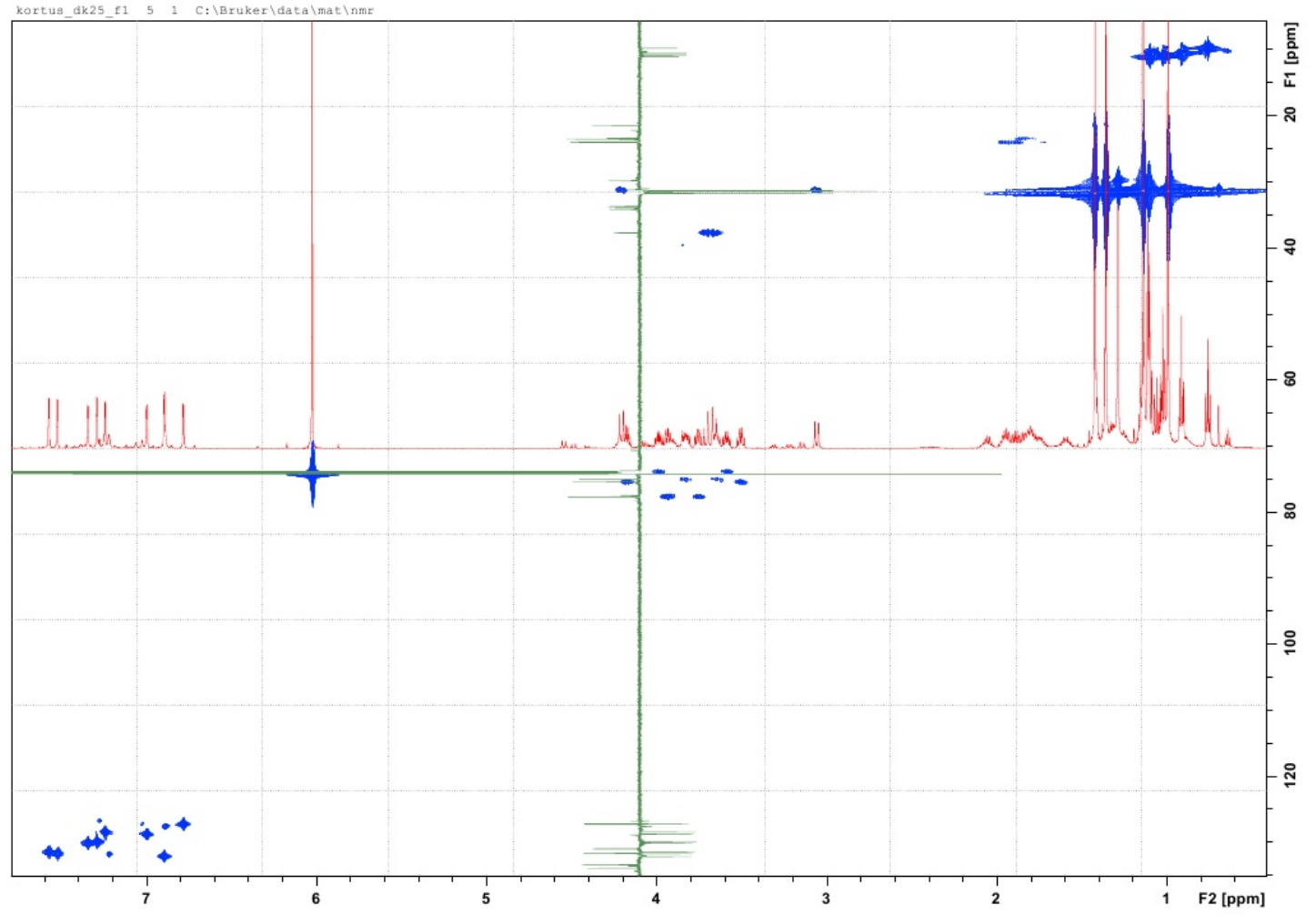

Figure S59: $\mathrm{HMQC}$ NMR of compound $6 \operatorname{Pr}\left(500.1 \mathrm{MHz}, \mathrm{C}_{2} \mathrm{D}_{2} \mathrm{Cl}_{4}\right)$ 


\subsection{Compound 6Bu}

192_Kortus_ESIpos_DK59_F2_1

5/17/2019 10:21:18 AM

192 Kortus ESIpos_DK59 F2 1 \#36-39 RT: 0.55-0.60 AV: 4 NL: 7.69E6

T: FTMS + c ESI Full ms [200.00-2000.00]

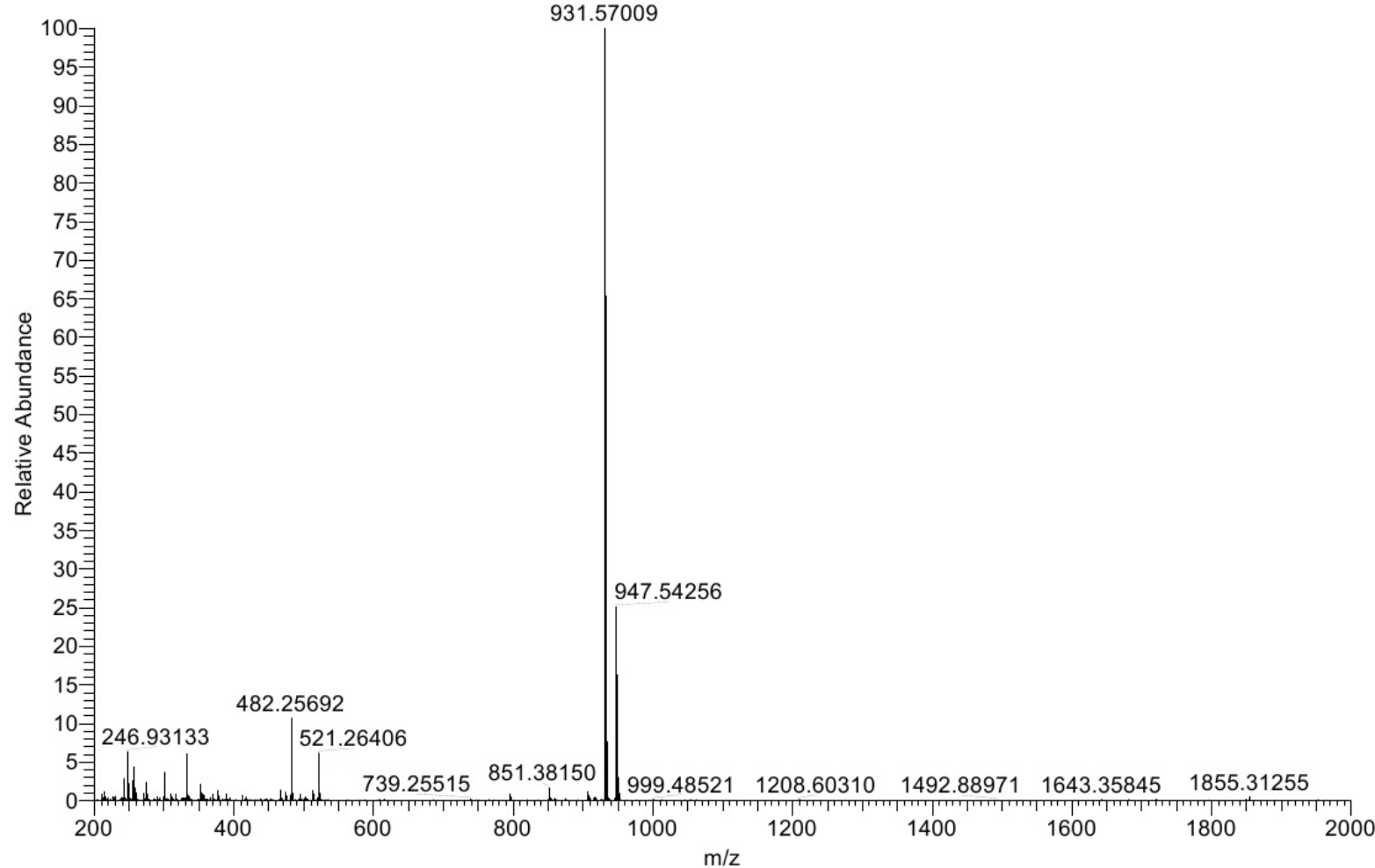

Figure S60: HRMS of compound 6Bu $\left(\mathrm{ESI}^{+}\right)$

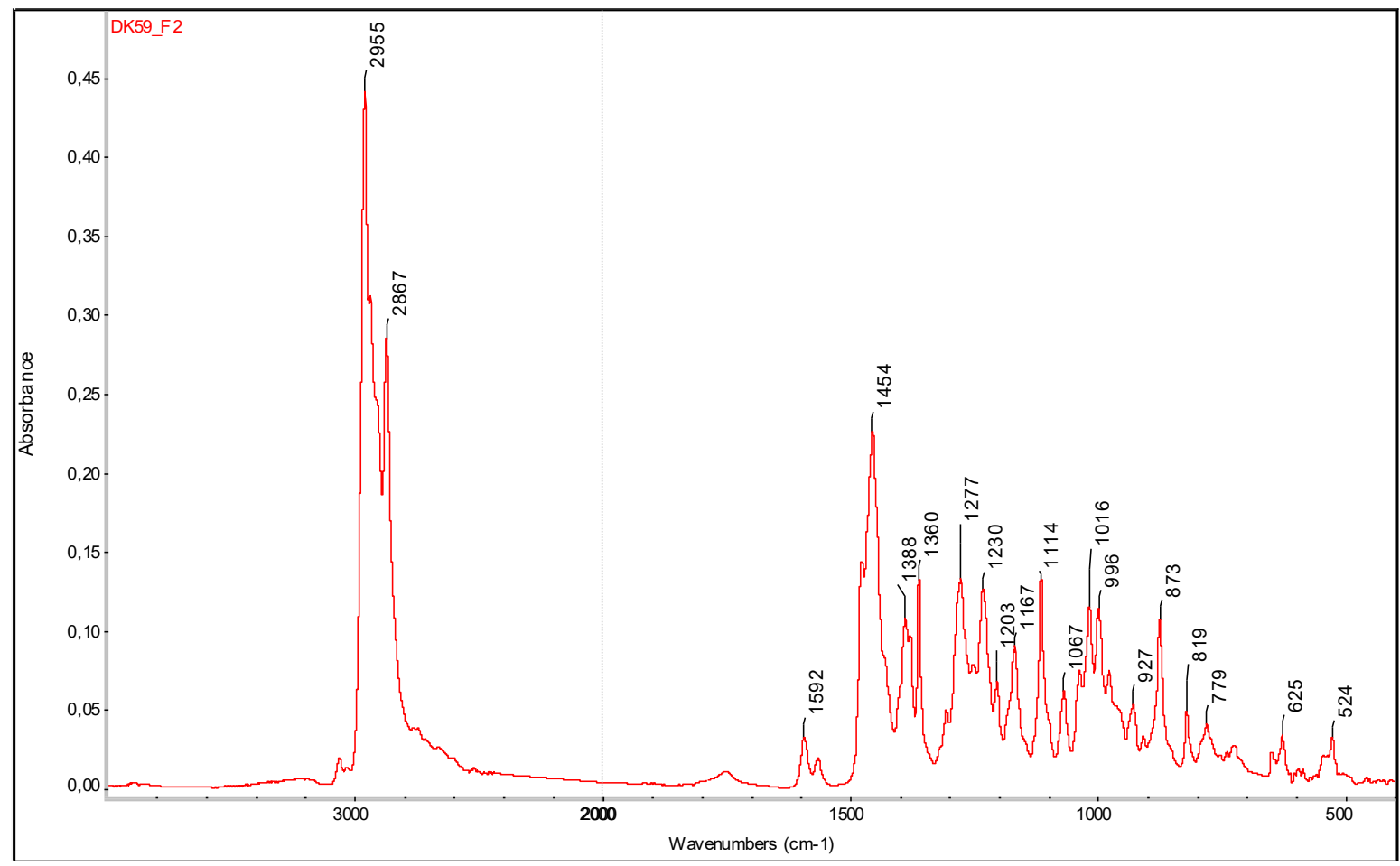

Figure S61: IR of compound 6Bu $(\mathrm{KBr})$ 


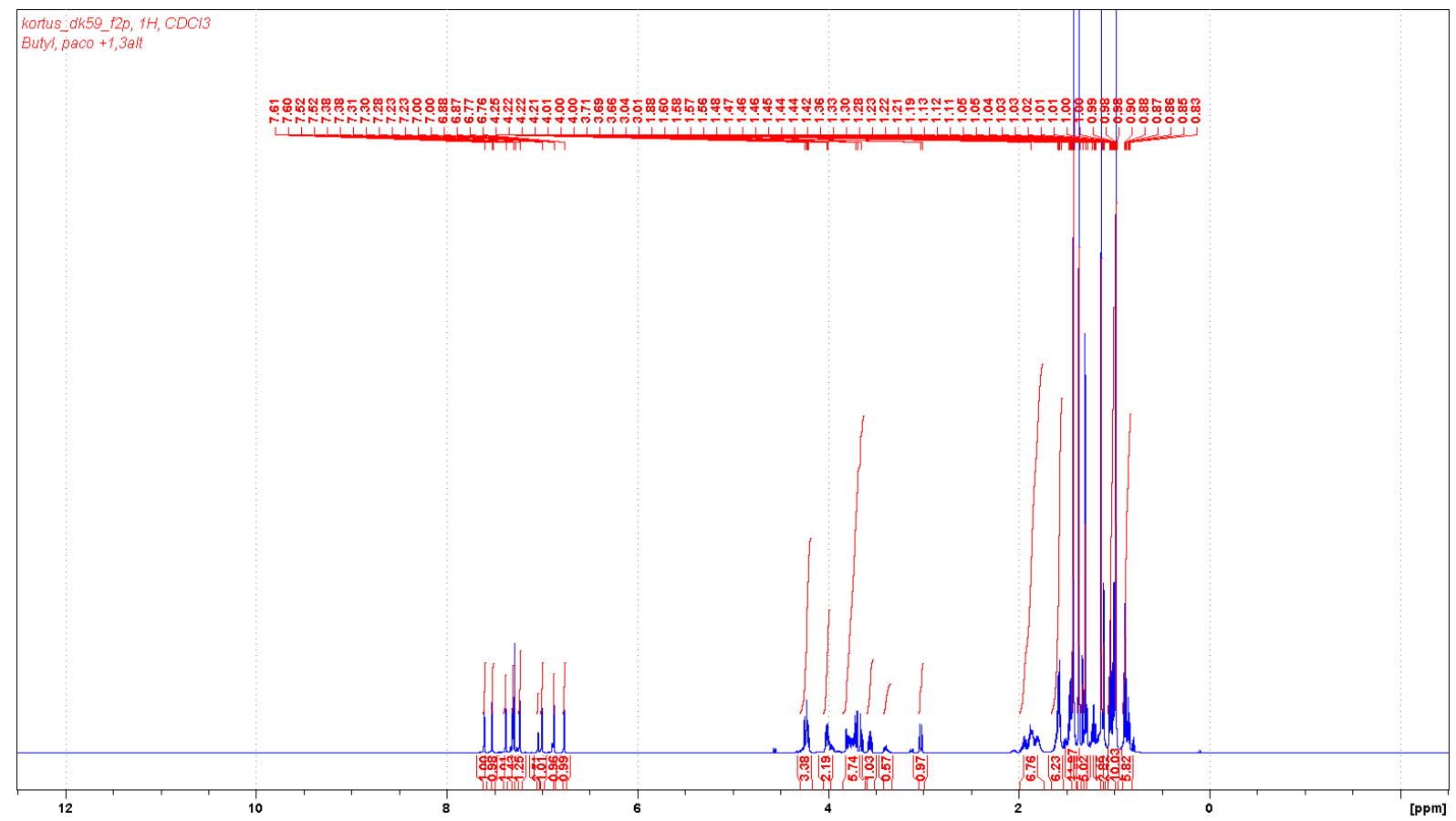

Figure S62: ${ }^{1} \mathrm{H}$ NMR of compound $6 \mathrm{Bu}\left(600.1 \mathrm{MHz}, \mathrm{CDCl}_{3}\right)$

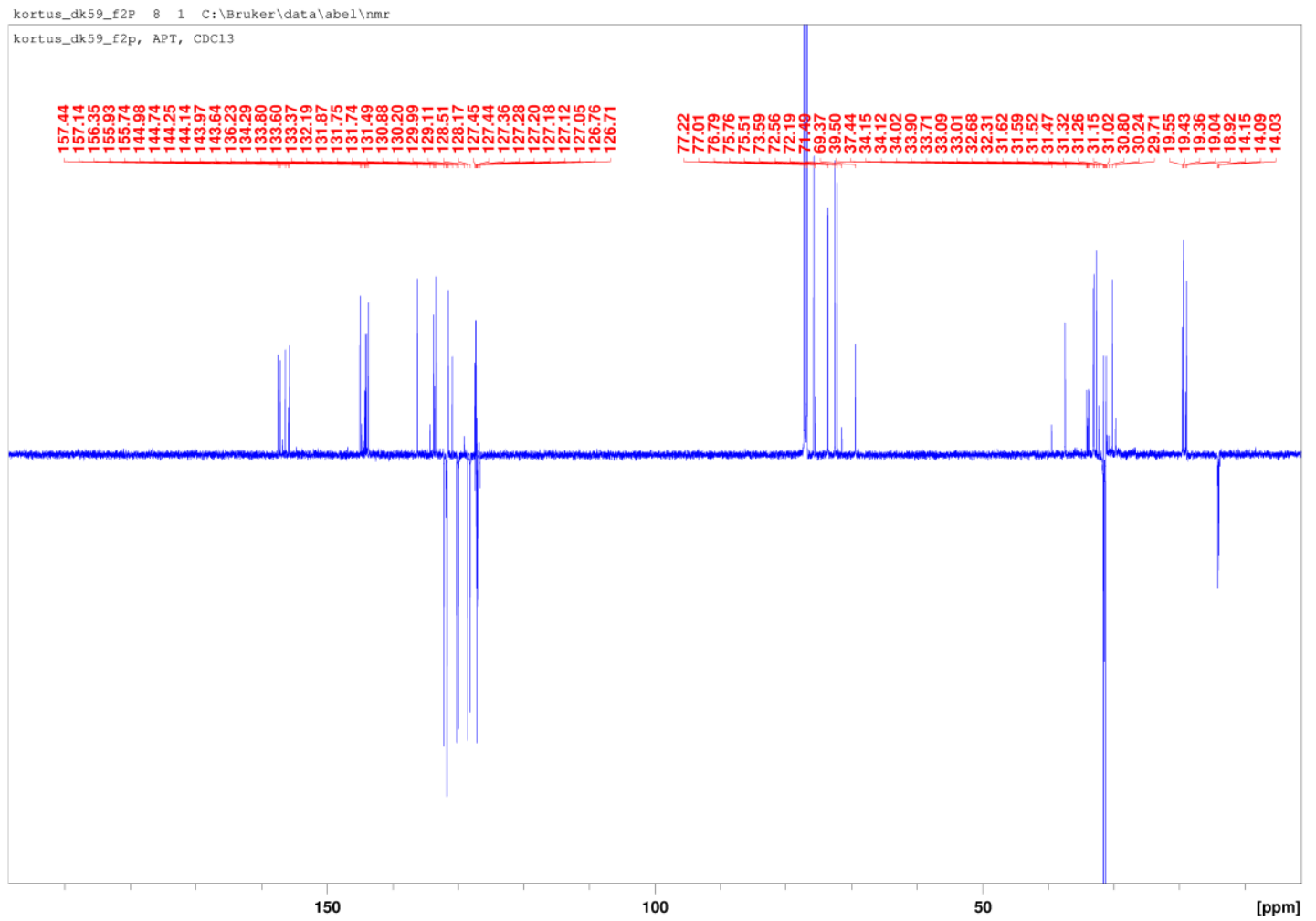

Figure S63: ${ }^{13} \mathrm{C}(\mathrm{APT}) \mathrm{NMR}$ of compound $6 \mathrm{Bu}\left(150.9 \mathrm{MHz}, \mathrm{CDCl}_{3}\right)$ 


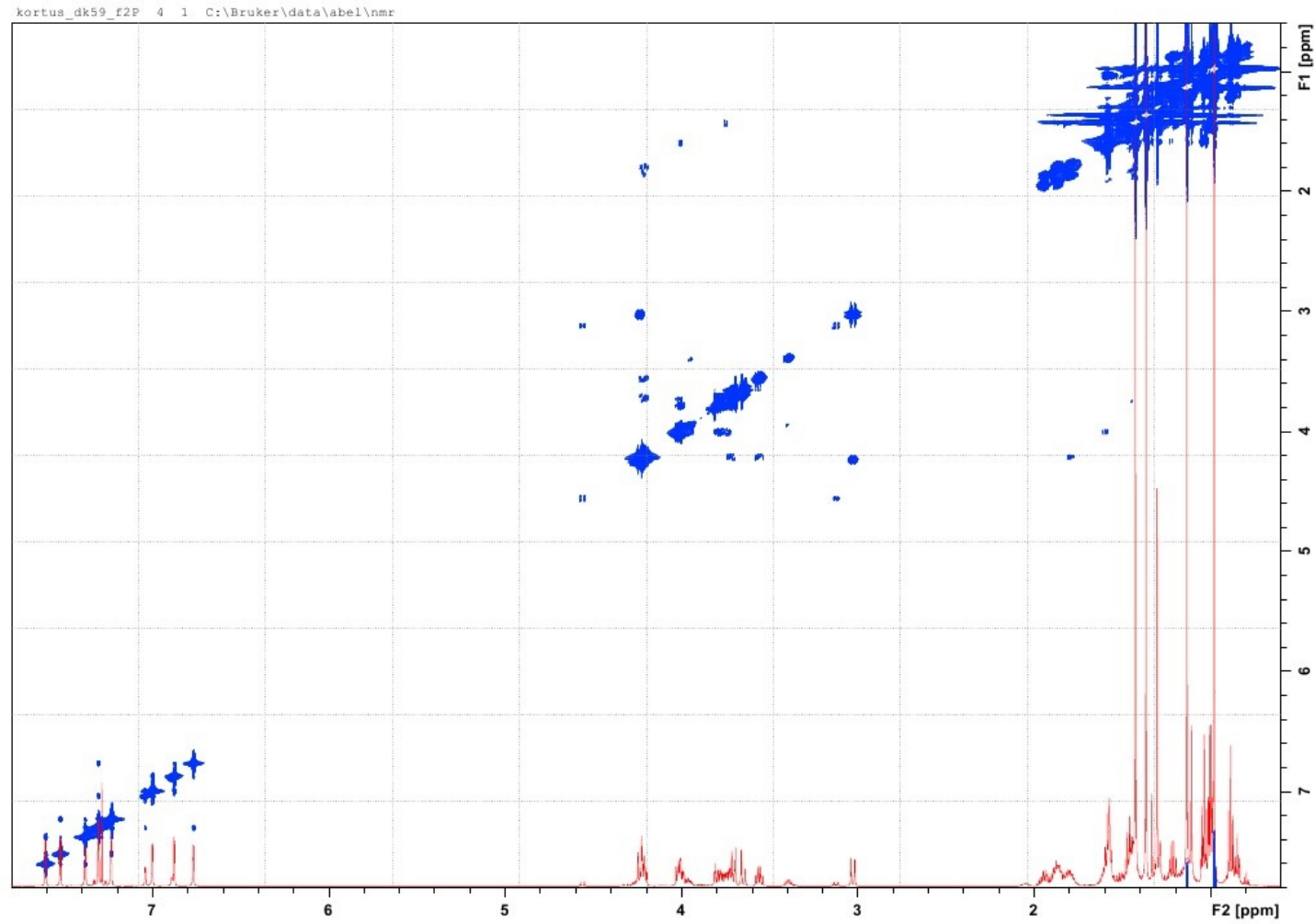

Figure S64: ${ }^{1} \mathrm{H}-{ }^{1} \mathrm{H}$ COSY NMR of compound $6 \mathrm{Bu}\left(600.1 \mathrm{MHz}, \mathrm{CDCl}_{3}\right)$

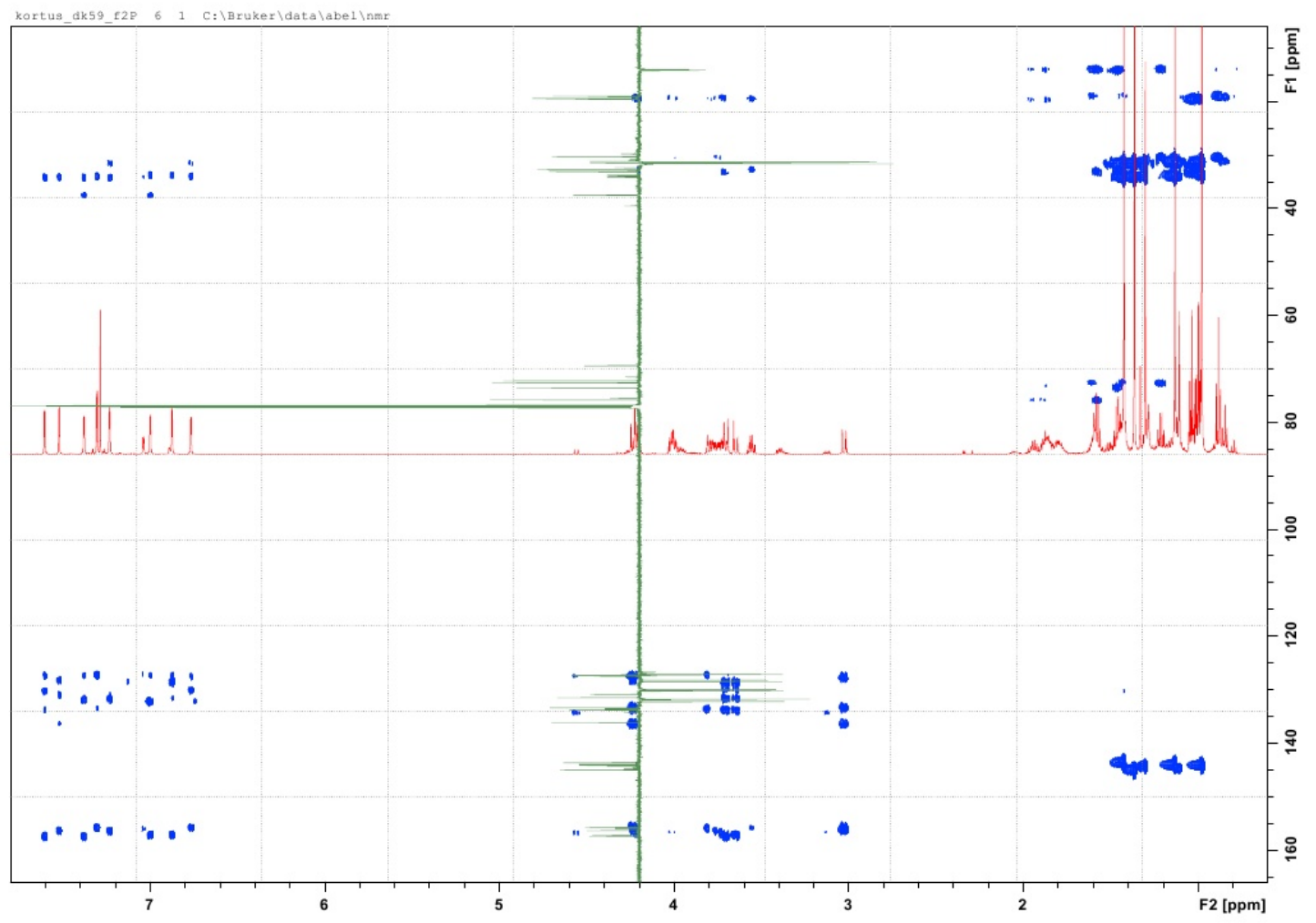

Figure S65: $\mathrm{HMBC}$ of compound $6 \mathrm{Bu}\left(600.1 \mathrm{MHz}, \mathrm{CDCl}_{3}\right)$ 


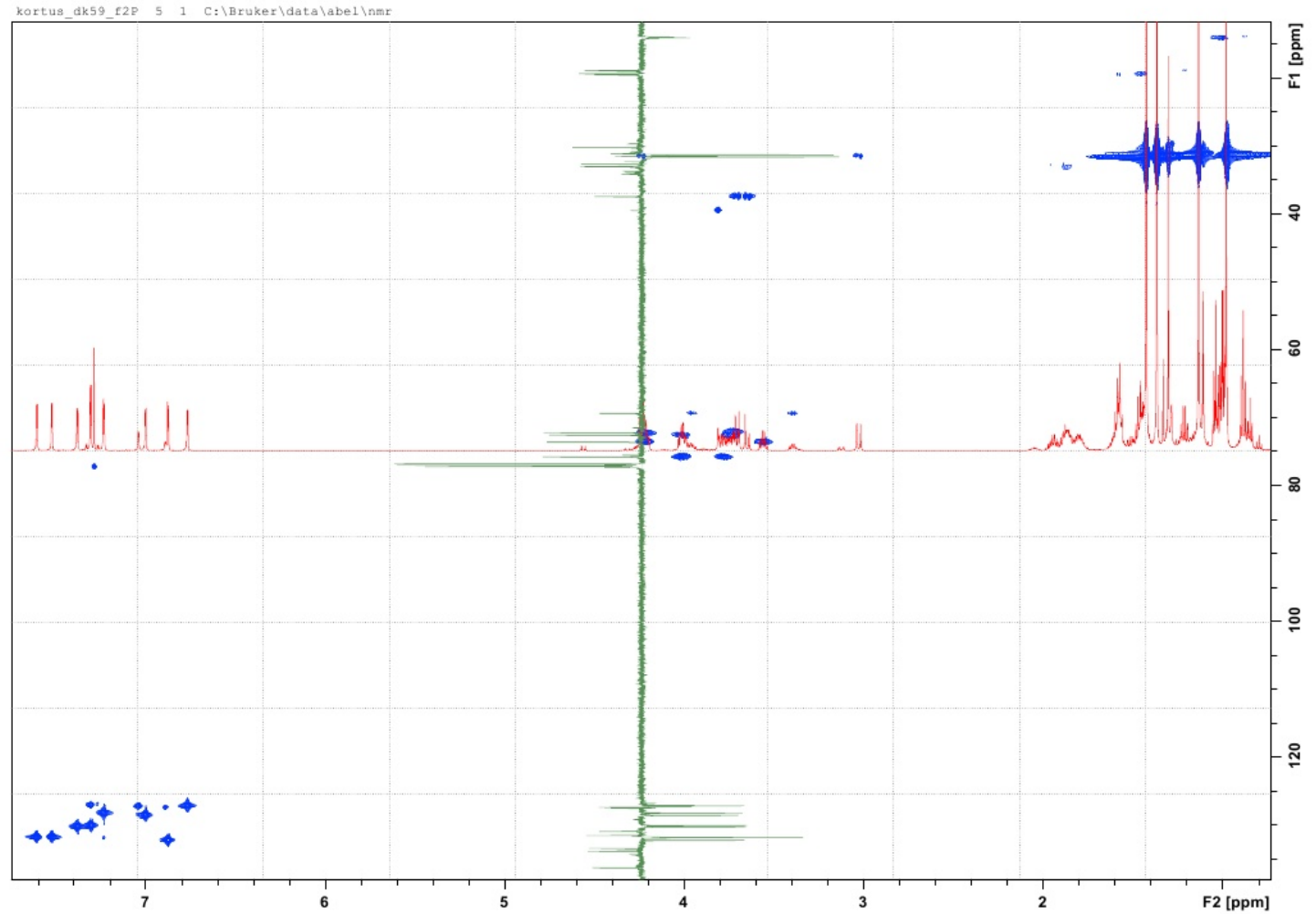

Figure S66: $\mathrm{HMQC}$ of compound $6 \mathrm{Bu}\left(600.1 \mathrm{MHz}, \mathrm{CDCl}_{3}\right)$ 


\section{CRYSTALLOGRAPHIC INFORMATION}

In all cases, slow evaporation of the solvents $\left(\mathrm{CH}_{2} \mathrm{Cl}_{2} / \mathrm{CH}_{3} \mathrm{OH}\right.$ mixtures $)$ was used to crystallise the products from their nearly saturated solutions at room temperature.

\subsection{Compound 3Pr}

The structure of 3Pr was measured using D8 VENTURE equipped with Photon CMOS detector with $\mathrm{Cu}-\mathrm{Ka}(\lambda=1.54178 \AA)$ radiation at $180 \mathrm{~K}$. The structure was in trigonal system, $P 3_{2} 21$ space group with lattice parameters $a=13.0211(4) \AA, b=13.0211(4) \AA, c=25.5008(7) \AA, \alpha=90^{\circ} B=90^{\circ} \gamma=120^{\circ}, Z=$ $2, V=3744.38(19) \AA^{3}, D_{c}=1.135 \mathrm{~g} / \mathrm{cm}^{3}, \mu(\mathrm{Cu}-\mathrm{K} \alpha)=1.287 \mathrm{~mm}^{-1}$. The data reduction and absorption correction were done with Apex3 software. The structure was solved by chargeflipping methods using Superflip software and refined by full matrix least squares on $F$ squared value using Crystals software to final values $R=0.0456$ and $w R=0.1084$ using 4576 independent reflections $\left(\Theta_{\max }=\right.$ $\left.68.218^{\circ}\right), 301$ parameters and 41 restraint. MCE software was used for visualization of residual electron density maps. According to common practice the hydrogen atoms attached to carbon atoms were place geometrically with $U_{\text {iso }}(\mathrm{H})$ in range 1.2-1.5 $U_{\text {eq }}$ of parent atom (C). The disordered functional groups were refined with restrained geometry and occupancy constrained to full for each atomic position. The structure was deposited into Cambridge Structural Database under number CCDC 1916009. 


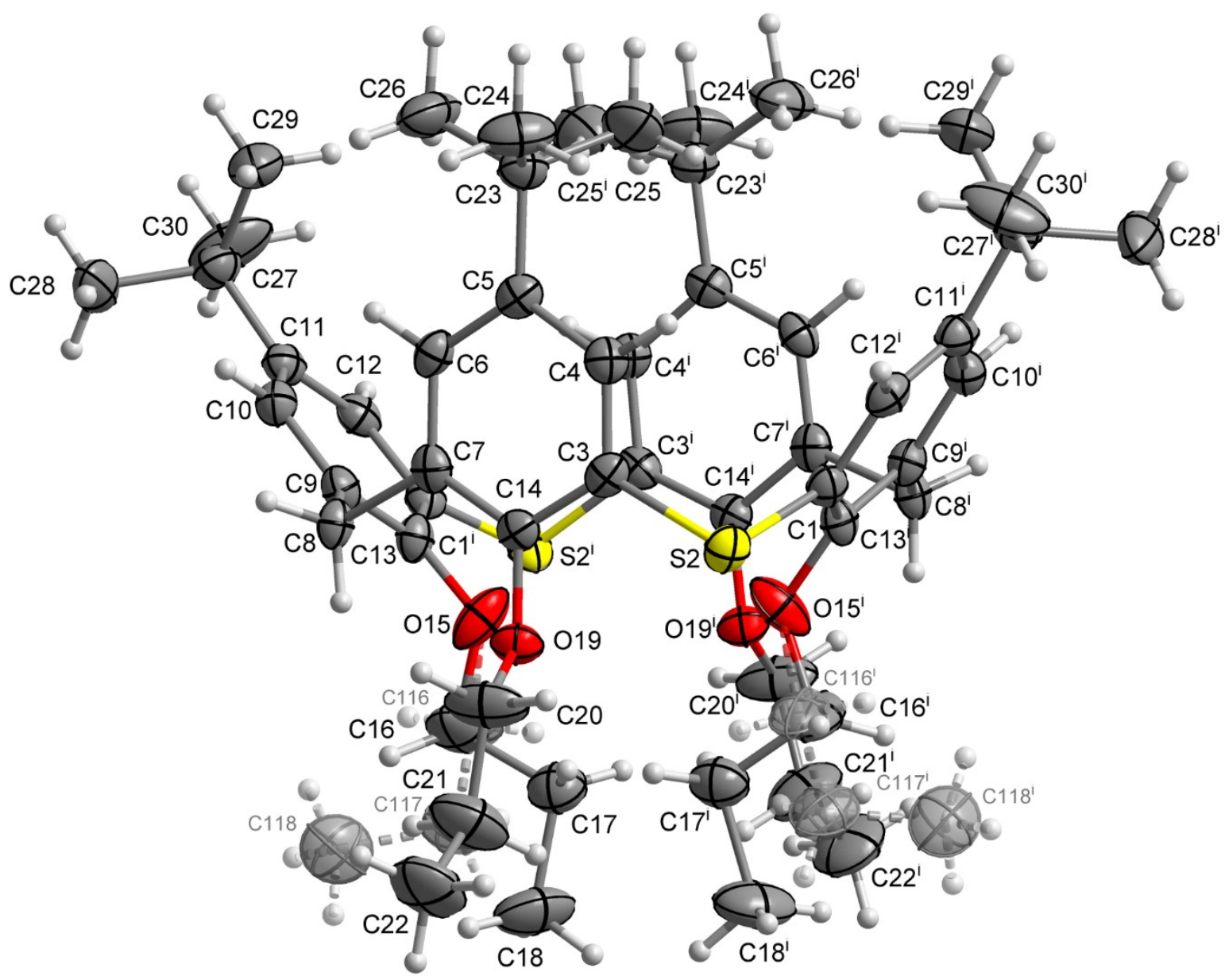

Figure S67: The numbering scheme of structure 3Pr with thermal ellipsoids drawn at $50 \%$ probability level. The weakly occupied atoms drawn as transparent with dashed bonds. Symmetry code: (i) $y, x, 1-$ z. 


\subsection{Compound 3Bu}

The structure of 3Bu was measured using D8 VENTURE equipped with Photon CMOS detector with Cu-Ka $\left(\lambda=1.54178 \AA\right.$ ) radiation at $180 \mathrm{~K}$. The structure was in trigonal system, $P 3_{2}$ space group with lattice parameters $a=13.8672(4) \AA, b=13.8672(4) \AA, c=24.9165(7) \AA, \alpha=90^{\circ} b=109.4783(16)^{\circ} \gamma=120^{\circ}, Z$ $=3, V=4149.5(3) \AA^{3}, D_{c}=1.092 \mathrm{~g} / \mathrm{cm}^{3}, \mu(\mathrm{Cu}-\mathrm{K} \alpha)=1.188 \mathrm{~mm}^{-1}$. The data reduction and absorption correction were done with Apex3 software. The structure was solved by chargeflipping methods using Superflip software and refined by full matrix least squares on $F$ squared value using Crystals software to final values $R=0.0740$ and $w R=0.2005$ using 9328 independent reflections $\left(\Theta_{\max }=\right.$ $\left.70.055^{\circ}\right), 633$ parameters and 205 restraint. The Flack parameter is $0.08(2)$. MCE software was used for visualization of residual electron density maps. According to common practice the hydrogen atoms attached to carbon atoms were place geometrically with $U_{\text {iso }}(H)$ in range 1.2-1.5 $U_{\text {eq }}$ of parent atom (C). The disordered functional groups were refined with restrained geometry and occupancy constrained to full for each atomic position. The structure was deposited into Cambridge Structural Database under number CCDC 1915508. 


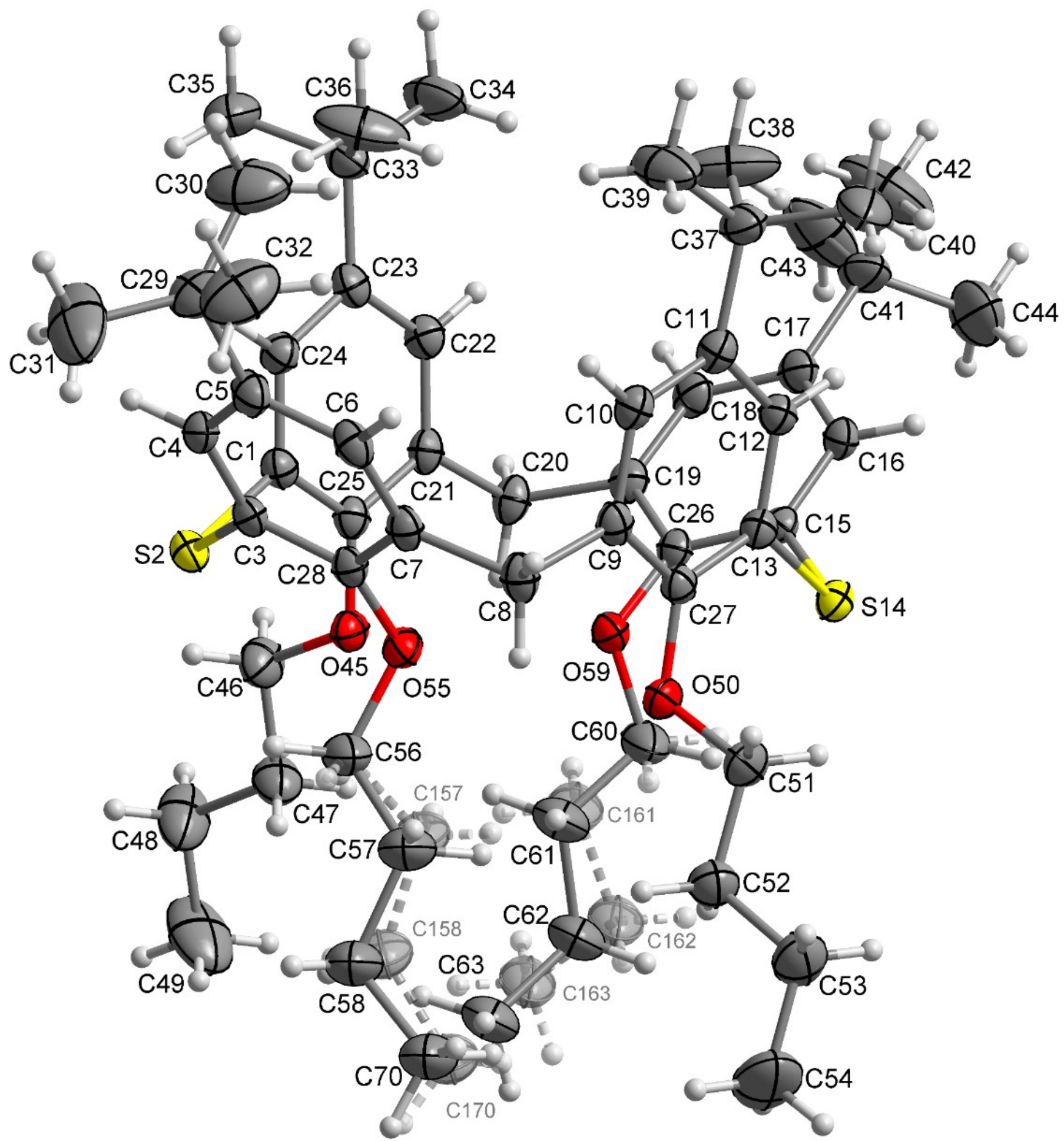

Figure S68: The numbering scheme of structure 3Bu with thermal ellipsoids drawn at $30 \%$ probability level. The weakly occupied atoms drawn as transparent with dashed bonds. 


\subsection{Compound 4Et}

The structure of 4Et was measured using D8 VENTURE equipped with Photon CMOS detector with Cu-Ka $\left(\lambda=1.54178 \AA\right.$ ) radiation at $180 \mathrm{~K}$. The structure was in monoclinic system, $P 2_{1} / n$ space group with lattice parameters $a=10.1236(3) \AA$, $b=22.2391(7) \AA, c=10.7810(3) \AA, \alpha=90^{\circ} b=107.3283(9)^{\circ} \gamma=$ $90^{\circ}, Z=2, V=2317.07(12) \AA^{3}, D_{c}=1.143 \mathrm{~g} / \mathrm{cm}^{3}, \mu(\mathrm{Cu}-\mathrm{K} \alpha)=1.355 \mathrm{~mm}^{-1}$. The data reduction and absorption correction were done with Apex3 software. The structure was solved by chargeflipping methods using Superflip software and refined by full matrix least squares on $F$ squared value using Crystals software to final values $R=0.0348$ and $w R=0.0889$ using 4180 independent reflections $\left(\Theta_{\max }=68.291^{\circ}\right), 292$ parameters and 37 restraint. MCE software was used for visualization of residual electron density maps. According to common practice the hydrogen atoms attached to carbon atoms were place geometrically with $U_{\text {iso }}(\mathrm{H})$ in range 1.2-1.5 $U_{\text {eq }}$ of parent atom $(\mathrm{C})$. The disordered functional groups were refined with restrained geometry and occupancy constrained to full for each atomic position. The structure was deposited into Cambridge Structural Database under number CCDC 1916008. 


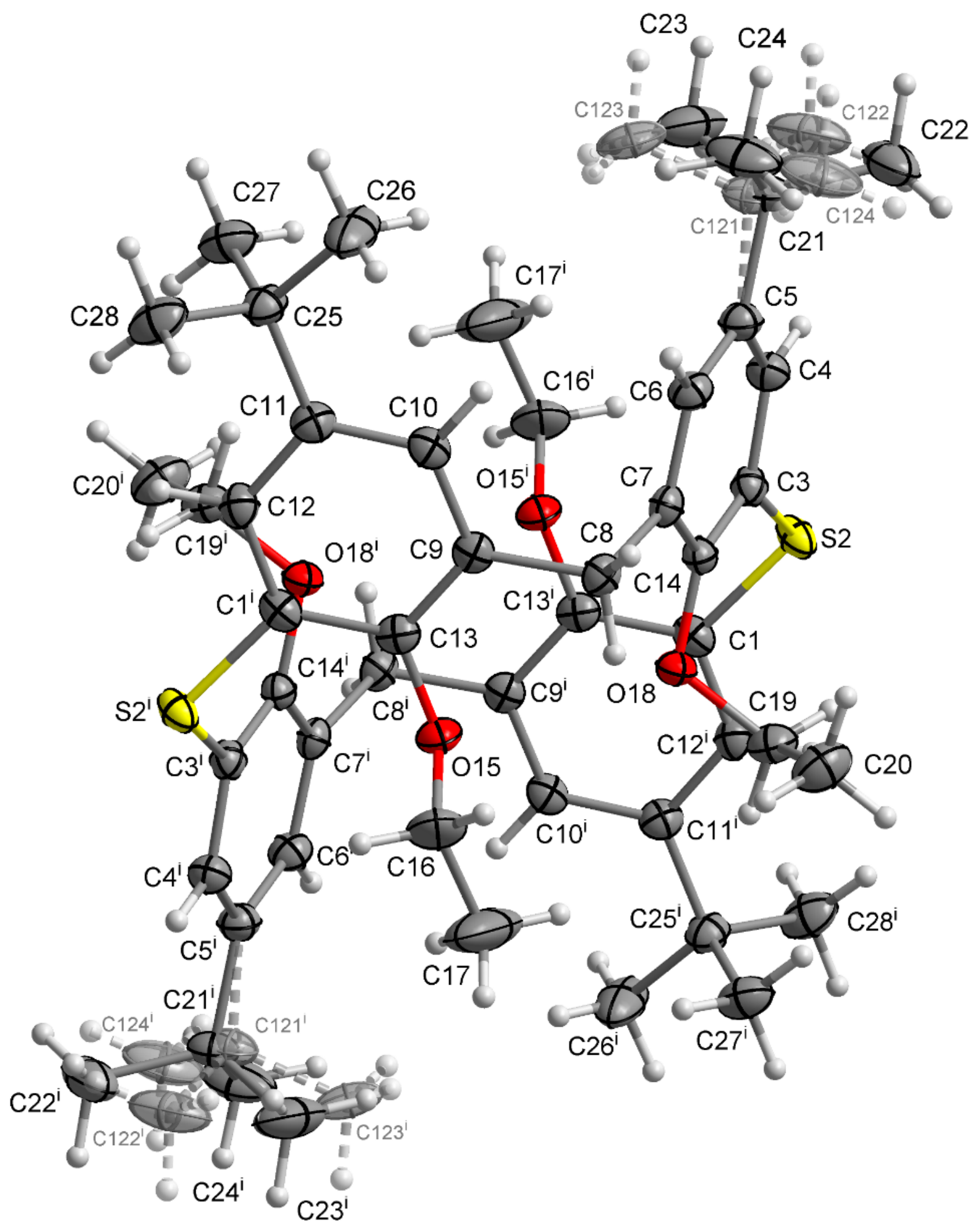

Figure S69: The numbering scheme of structure 4Et with thermal ellipsoids drawn at $50 \%$ probability level. The weakly occupied atoms drawn as transparent with dashed bonds. Symmetry code: (i) $-x, 1-$ $y,-z$. 


\subsection{Compound 4Pr}

The structure of 4Pr was measured using D8 VENTURE equipped with Photon CMOS detector with Cu-Ka $(\lambda=1.54178 \AA$ ) radiation at $180 \mathrm{~K}$. The structure was in tetragonal system, $P-1$ space group with lattice parameters $a=9.3862(3) \AA, b=14.2171(5) \AA, c=19.5174(7) \AA, \alpha=100.4797(10)^{\circ} b=$ 99.0902 (10) $)^{\circ} \gamma=93.0281(10)^{\circ}, Z=2, V=2519.94(15) \AA^{3}, D_{c}=1.125 \mathrm{~g} / \mathrm{cm}^{3}, \mu(\mathrm{Cu}-\mathrm{K} \alpha)=1.275 \mathrm{~mm}^{-1}$. The data reduction and absorption correction were done with Apex 3 software. The structure was solved by chargeflipping methods using Superflip software and refined by full matrix least squares on $F$ squared value using Crystals software to final values $R=0.0383$ and $w R=0.0975$ using 9094 independent reflections $\left(\Theta_{\max }=67.256^{\circ}\right), 542$ parameters and no restraint. MCE software was used for visualization of residual electron density maps. According to common practice the hydrogen atoms attached to carbon atoms were place geometrically with $U_{\text {iso }}(H)$ in range 1.2-1.5 $U_{\text {eq }}$ of parent atom (C). The structure was deposited into Cambridge Structural Database under number CCDC 1915506. 


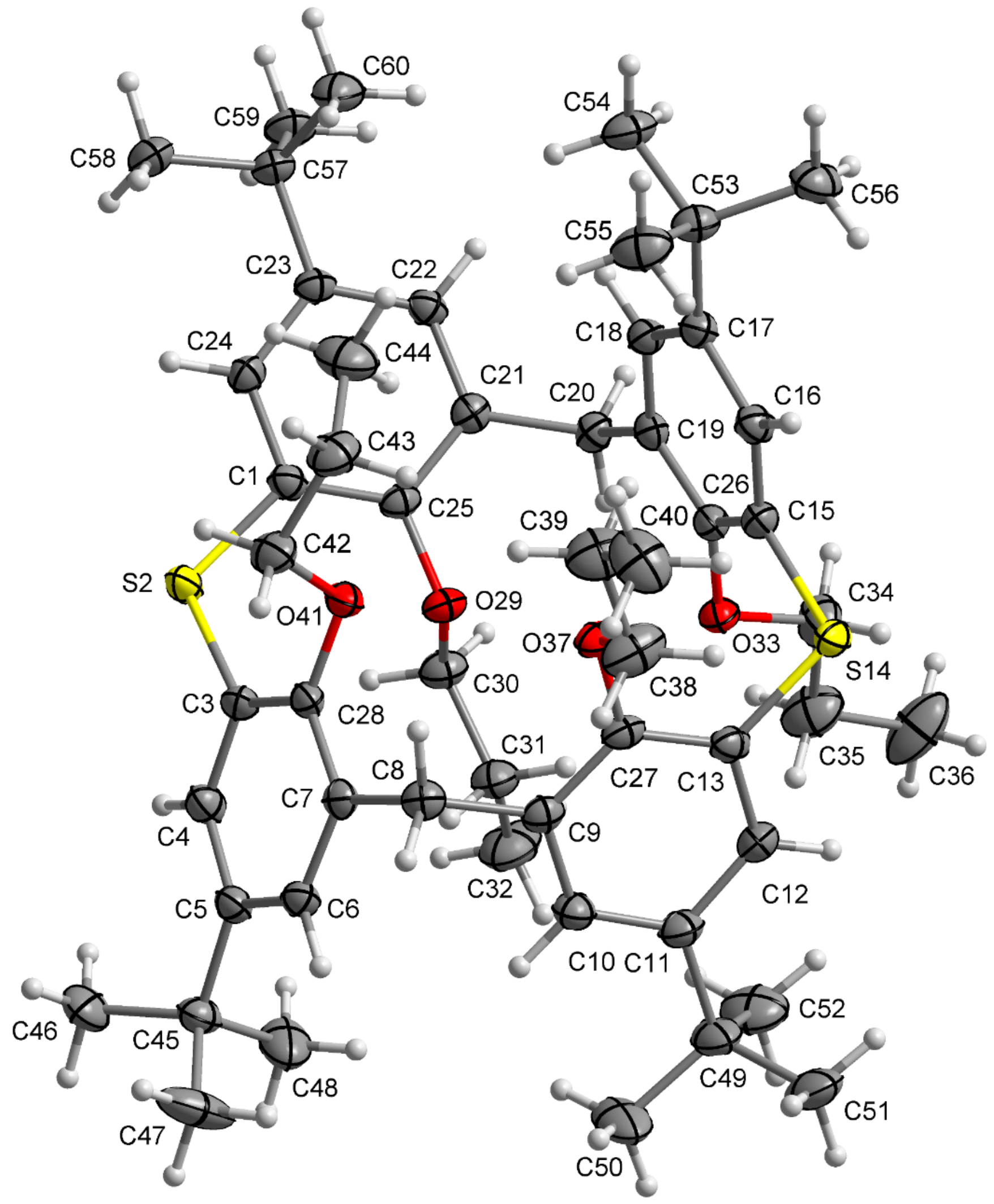

Figure S70: The numbering scheme of structure 4Pr with thermal ellipsoids drawn at $50 \%$ probability level. The weakly occupied atoms drawn as transparent with dashed bonds. 


\subsection{Compound 4Bu}

The structure of 4Bu was measured using D8 VENTURE equipped with Photon CMOS detector with Cu-Ka $(\lambda=1.54178 \AA)$ radiation at $180 \mathrm{~K}$. The structure was in trigonal system, $P 2_{1}$ space group with lattice parameters $a=10.0198(3) \AA, b=40.3929(11) \AA, c=13.8991(4) \AA, \alpha=90^{\circ} b=100.8552(10)^{\circ} \gamma=$ $90^{\circ}, Z=4, V=5524.7(3) \AA^{3}, D_{c}=909.37 \mathrm{~g} / \mathrm{cm}^{3}, \mu(\mathrm{Cu}-\mathrm{K} \alpha)=1.189 \mathrm{~mm}^{-1}$. The data reduction and absorption correction were done with Apex3 software. The structure was solved by chargeflipping methods using Superflip software and refined by full matrix least squares on $F$ squared value using Crystals software to final values $R=0.0707$ and $w R=0.1941$ using 20357 independent reflections $\left(\Theta_{\max }=72.116^{\circ}\right), 1275$ parameters and 361 restraint. MCE software was used for visualization of residual electron density maps. According to common practice the hydrogen atoms attached to carbon atoms were place geometrically with $U_{\text {iso }}(\mathrm{H})$ in range 1.2-1.5 $U_{\text {eq }}$ of parent atom (C). The disordered functional groups were refined with restrained geometry and occupancy constrained to full for each atomic position. The structure was deposited into Cambridge Structural Database under number CCDC 1915509. 


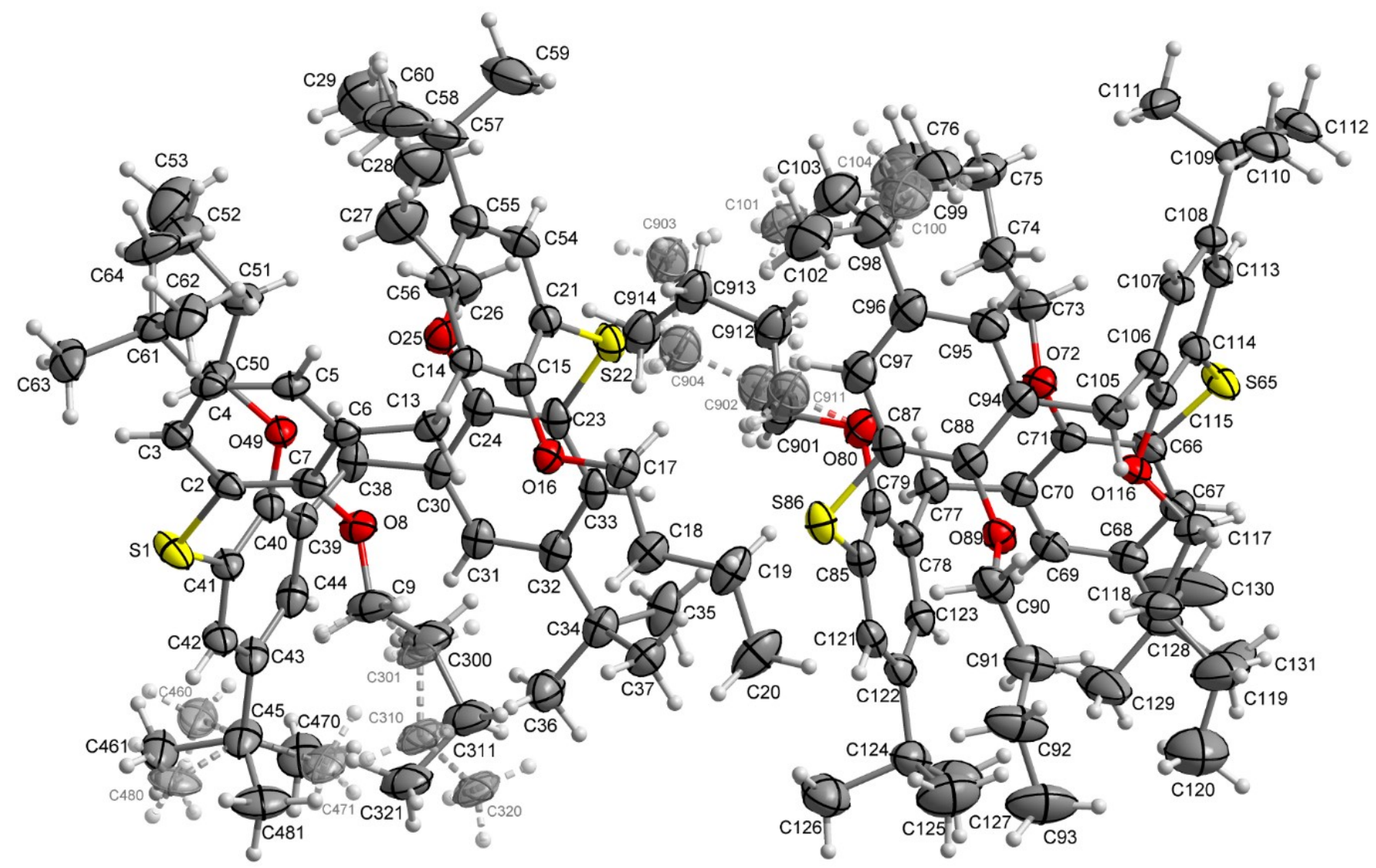

Figure S71: The numbering scheme of structure 4Bu with thermal ellipsoids drawn at $30 \%$ probability level. The weakly occupied atoms drawn as transparent with dashed bonds. 


\subsection{Compound 5Pr}

The structure of 5Pr was measured using D8 VENTURE equipped with Photon CMOS detector with Cu-Ka $(\lambda=1.54178 \AA$ ) radiation at $180 \mathrm{~K}$. The structure was in tetragonal system, $P-1$ space group with lattice parameters $a=10.4974(4) \AA, b=13.0623(5) \AA, c=19.5386(7) \AA, \alpha=108.2731(12)^{\circ} b=$ $93.3489(12)^{\circ} \gamma=96.5858(13)^{\circ}, Z=2, V=2514.57(16) \AA^{3}, D_{c}=1.127 \mathrm{~g} / \mathrm{cm}^{3}, \mu(\mathrm{Cu}-\mathrm{K} \alpha)=1.277 \mathrm{~mm}^{-1}$. The data reduction and absorption correction were done with Apex 3 software. The structure was solved by chargeflipping methods using Superflip software and refined by full matrix least squares on $F$ squared value using Crystals software to final values $R=0.0450$ and $w R=0.1151$ using 8925 independent reflections $\left(\Theta_{\max }=68.324^{\circ}\right), 655$ parameters and 116 restraint. MCE software was used for visualization of residual electron density maps. According to common practice the hydrogen atoms attached to carbon atoms were place geometrically with $U_{\text {iso }}(H)$ in range 1.2-1.5 $U_{\text {eq }}$ of parent atom $(\mathrm{C})$. The disordered functional groups were refined with restrained geometry and occupancy constrained to full for each atomic position. The structure was deposited into Cambridge Structural Database under number CCDC 1916010. 




Figure S72: The numbering scheme of structure 5Pr with thermal ellipsoids drawn at $50 \%$ probability level. The weakly occupied atoms drawn as transparent with dashed bonds. 


\subsection{Compound 6Pr}

The structure of 6Pr was measured using D8 VENTURE equipped with Photon CMOS detector with $\mathrm{Cu}-\mathrm{Ka}(\lambda=1.54178 \AA)$ radiation at $180 \mathrm{~K}$. The structure was in monoclinic system, $C 2 / \mathrm{c}$ space group with lattice parameters $a=51.068(2) \AA, b=10.7010(4) \AA, c=20.7984(9) \AA, \alpha=90^{\circ} B=109.4783(16)^{\circ} \gamma=$ $90^{\circ}, Z=4, V=10715.3(8) \AA^{3}, D_{c}=1.058 \mathrm{~g} / \mathrm{cm}^{3}, \mu(\mathrm{Cu}-\mathrm{K} \alpha)=1.199 \mathrm{~mm}^{-1}$. The data reduction and absorption correction were done with Apex3 software. The structure was solved by chargeflipping methods using Superflip software and refined by full matrix least squares on $F$ squared value using Crystals software to final values $R=0.0617$ and $w R=0.1711$ using 8278 independent reflections $\left(\Theta_{\max }=70.067^{\circ}\right), 690$ parameters and 206 restraint. MCE software was used for visualization of residual electron density maps. According to common practice the hydrogen atoms attached to carbon atoms were place geometrically with $U_{\text {iso }}(\mathrm{H})$ in range 1.2-1.5 $U_{\text {eq }}$ of parent atom (C). The disordered functional groups were refined with restrained geometry and occupancy constrained to full for each atomic position. The structure was deposited into Cambridge Structural Database under number CCDC 1915507. 


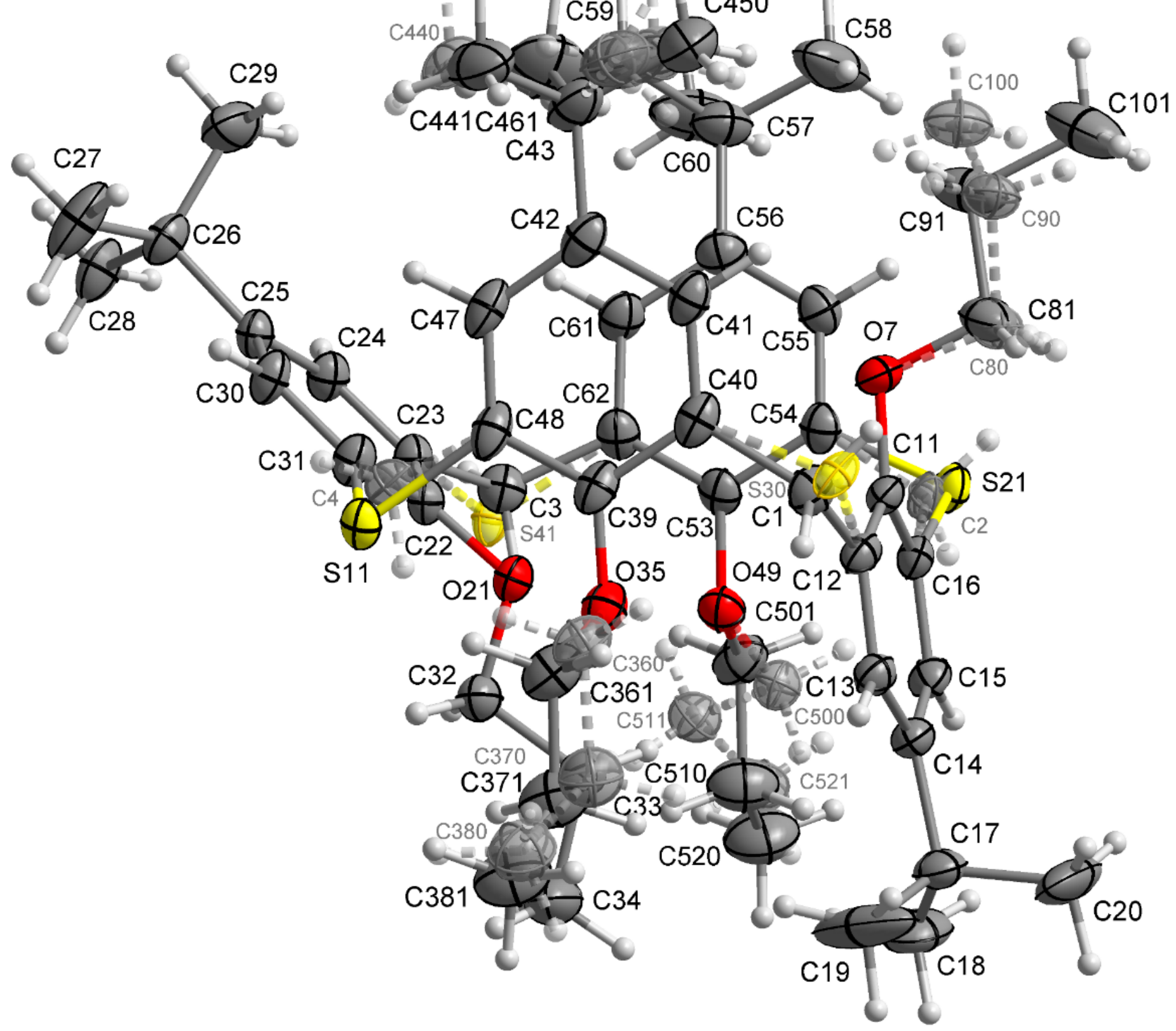

Figure S73: The numbering scheme of structure 6Pr with thermal ellipsoids drawn at $30 \%$ probability level. The weakly occupied atoms drawn as transparent with dashed bonds.. 


\section{RESULTS OF QUANTUM CHEMICAL CALCULATIONS}

\subsection{Compound 3Et}

Final single point energy: -3042.563716117755

\begin{tabular}{|c|c|}
\hline C & -2.70838016014073 \\
\hline $\mathrm{C}$ & -2.51669283863142 \\
\hline $\mathrm{C}$ & -2.61903834598540 \\
\hline C & -2.86304152216627 \\
\hline C & -2.94603190250629 \\
\hline C & -2.85899020989140 \\
\hline $\mathrm{H}$ & -2.48616688830605 \\
\hline $\mathrm{H}$ & -3.09134268667044 \\
\hline $\mathrm{C}$ & 2.62652081300159 \\
\hline C & 2.90061934050148 \\
\hline $\mathrm{C}$ & 2.56503201303616 \\
\hline $\mathrm{C}$ & 2.96481834215995 \\
\hline $\mathrm{C}$ & 2.72563173257140 \\
\hline $\mathrm{H}$ & 2.36992746393008 \\
\hline $\mathrm{C}$ & 2.89074742376241 \\
\hline $\mathrm{H}$ & 2.97707074711534 \\
\hline $\mathrm{H}$ & -2.51443425837724 \\
\hline $\mathrm{C}$ & -1.52658946733324 \\
\hline $\mathrm{C}$ & -0.38833906040695 \\
\hline $\mathrm{C}$ & -1.44150754758638 \\
\hline $\mathrm{C}$ & 0.85277920514090 \\
\hline C & -0.18433636879261 \\
\hline $\mathrm{C}$ & 0.97930203689977 \\
\hline $\mathrm{H}$ & 1.76553148681703 \\
\hline $\mathrm{C}$ & -0.82823234710873 \\
\hline $\mathrm{C}$ & -0.79657503833272 \\
\hline $\mathrm{C}$ & 0.38772304882026 \\
\hline C & 0.39537167333074 \\
\hline $\mathrm{H}$ & -1.74414302245835 \\
\hline $\mathrm{C}$ & 1.58976324539651 \\
\hline $\mathrm{C}$ & 1.57930825028661 \\
\hline $\mathrm{H}$ & 2.52951524608057 \\
\hline 0 & -2.69916653083129 \\
\hline O & -0.04612894407352 \\
\hline O & 3.05430179149977 \\
\hline O & 0.34327206156517 \\
\hline $\mathrm{C}$ & -2.15082705877408 \\
\hline $\mathrm{H}$ & -2.10105701022593 \\
\hline $\mathrm{H}$ & -2.93483875113223 \\
\hline S & -2.97763165315061 \\
\hline $\mathrm{C}$ & 2.34489688866736 \\
\hline $\mathrm{H}$ & 3.10919055796462 \\
\hline $\mathrm{H}$ & 2.40992652246308 \\
\hline S & 3.17792316514482 \\
\hline $\mathrm{C}$ & 2.75223106862234 \\
\hline $\mathrm{C}$ & 4.04083014623326 \\
\hline $\mathrm{H}$ & 4.08884827602541 \\
\hline $\mathrm{H}$ & 4.07951329626889 \\
\hline $\mathrm{H}$ & 4.9314 \\
\hline
\end{tabular}

$-0.82298190956083$

$-1.50191194015444$

$-0.78638094826073$

0.59115783081243

1.25559468482112

0.57196454141496

$-1.33394392168436$

2. 32620787575526

1.25377445002588

$-0.12545672453141$

1.97287195639694

$-0.76102459983318$

1.37969153241217

3.03733565638337

$-0.00520496206996$

$-0.53182342440668$

4.28917886605327

3. 85623016597370

4.66026529210971

2.46691294378737

4.00892294089402

1.85066699863159

2.62896639952914

4.59172900246735

$-3.24157079263844$

$-3.77408039331790$

$-2.92662440278229$

$-3.98765717833101$

$-4.04049247195445$

$-3.05082126822281$

$-3.59026342781254$

$-3.67941107231399$

$-1.51865859976237$

0.50476252445545

$-0.84848554202954$

$-2.43029346523609$

$-2.98311332494990$

$-3.36255522295519$

$-3.54172667354375$

1. 52222152850046

1.96232382440459

2. 72619991820607

1.23723264168566

$-2.55127135361676$

2. 24933377047233

3.10562800830564

3. 73999776632913

3.75616740680923

2. 47043120313335
0.73817796690020

$-0.47935458315001$

$-1.67084478531540$

$-1.71450497436699$

$-0.49056505509242$

0.72635601001589

$-2.59672634661214$

$-0.45192051933043$

0.83173792275495

0.77750117509834

$-0.36017550506818$

$-0.47131993988054$

$-1.61800554798873$

$-0.29298736874371$

$-1.64577277975515$

$-2.58574624638074$

1. 98116035980679

2.05881833245773

1. 98978698323966

2. 21289309091603

2. 02723715874293

2.33425133325652

2.18016660245849

1. 94772255269467

$-1.22402782632266$

$-2.51187585900331$

$-0.58645552072781$

$-3.21898964814207$

$-2.96954831627389$

$-1.30408591664762$

$-2.59668225416628$

$-3.10546229415616$

1.92673104020889

2.51286721856398

1.93941417542388

0.68451581903996

$-0.52062452338385$

0.49728244531251

$-1.04224743174501$

2. 26048112814004

2.15465062021247

2.33345526675085

2. 96248033666851

$-0.60816521102182$

$-2.88854530301293$

$-2.87604949393134$

$-3.76789439651935$

$-1.99810676512218$

$-2.86150111466983$ 
1.40294834176915

0.76690494885727

0.76443643126491

2.06285697408414

3.18684258872112

2. 61437992956442

3. 88234052265363

3. 78228772021447

$-4.61571486859778$

$-4.74778940335544$

$-3.77539799561390$

$-5.38205519275802$

$-5.20505336204732$

$-3.74105062573529$

$-3.65737461011204$

$-2.73091281084372$

$-4.17585258166066$

$-6.03123150453704$

$-6.49412557736466$

$-6.67446835312272$

$-6.00217913214651$

1.30386543202732

2. 83722315782558

3.30146622569774

3.20998613903466

3.18028719286044

0.89237780616872

1.11651918056537

$-0.17383110854399$

1.43915428722653

0.89676902861466

1.19091280457083

1.38077286591777

$-0.18480644771631$

6.19256059517022

6.71461529094435

7.80526510364150

6.31845406213233

6.45770811781883

6.84771288040018

6.56353380115443

6.54836062129893

7.93907987669897

6.62866611071917

6.33408724339930

6.17943324108915

7.71726350856626

$-0.04628665951108$

$-1.04343129169935$

0.52943858155098

$-0.09228923258545$

$-0.58540180056257$

$-0.65165894647531$

0.91211043974253

$-2.23673335039602$

$-2.97221575437964$

$-2.78094596878293$

$-1.35154856779507$
$-4.17480468367183$

$-4.25050608074427$

$-4.23637071637901$

$-5.04741469577957$

$-2.92473957661042$

$-2.89593682387819$

$-2.08243239887987$

$-3.84345288613139$

$-4.62454429848866$

$-5.24517845065003$

$-5.36801711241333$

$-4.64015674800047$

$-6.23570546967408$

$-5.56502377107030$

$-5.21029652567112$

$-5.64380419092285$

$-6.56917965011791$

$-4.53432183260776$

$-5.52548808090194$

$-3.88033817062970$

$-4.13747674216844$

$-3.06297542065435$

$-2.92066383012985$

$-3.90474016532534$

$-2.29236868923291$

$-2.49281917723002$

$-4.07972221528044$

$-3.69906843734636$

$-4.31348674458717$

$-5.01748766072957$

$-3.61900324628396$

$-2.93079066360831$

$-4.58370112090575$

$-3.76495423113835$

1.85095851352356

1.83694576423941

1.75067024622657

0.98950546436261

2.75607130221363

3.04360103004732

3.99157734479683

3.08579458989451

2. 96011759566991

0.53121603414780

0.49894706994835

$-0.33116434599447$

0.42166712675255

3.79135368499603

3.55983046884096

4.19538122757548

4.77206839745309

5.69688170213597

4. 34952275460835

5.03245014895532

2.24670034331976

2.99399427482461

1.36143442670451

2. 79624951875347 


$\begin{array}{ll}\mathrm{H} & -5.87975579423647 \\ \mathrm{H} & -4.68600774639619 \\ \mathrm{H} & -5.34730124073453 \\ \mathrm{C} & 0.88370849231370 \\ \mathrm{H} & 1.18861288934249 \\ \mathrm{H} & 1.78224838026844 \\ \mathrm{C} & -0.13737149563559 \\ \mathrm{H} & 0.29687395671546 \\ \mathrm{H} & -1.02944102286009 \\ \mathrm{H} & -0.43252353344688 \\ \mathrm{C} & 4.32852615927628 \\ \mathrm{H} & 4.59602154892496 \\ \mathrm{H} & 4.15135571041392 \\ \mathrm{C} & 5.45101960607527 \\ \mathrm{H} & 6.36858615828609 \\ \mathrm{H} & 5.20273660393849 \\ \mathrm{H} & 5.65320727971188\end{array}$

$-1.97188170454494$

$-0.81801309510996$

$-0.61774945184665$

$-3.25506704520114$

$-2.54575784750895$

$-3.76306212803166$

$-4.24907230270709$

$-4.80821734177223$

$-3.73041906885765$

$-4.97236727507574$

$-0.71373579105858$

0.34716402447178

$-1.08464066257184$

$-1.49822446193979$

$-1.38842127625547$

$-2.55929929870997$

$-1.13005833870468$
3.06185866815309

3. 68888879298036

2.05709144427865

1. 74294209084054

2.50956733338707

1.38782929369726

2.27733554000110

3.11304774863327

2. 63527183214928

1.51165624562390

2.60137140315426

2.67319884654388

3. 61347370010344

1. 93587542232054

2. 52328850221876

1.87285438703888

0.92738148059041

\subsection{Compound 3Pr}

Final single point energy: -3199.743078641272

$\begin{array}{ll}\mathrm{C} & -2.70928488577777 \\ \mathrm{C} & -2.52566816071479 \\ \mathrm{C} & -2.63442338997263 \\ \mathrm{C} & -2.87756944412133 \\ \mathrm{C} & -2.95336443954136 \\ \mathrm{C} & -2.85932683995987 \\ \mathrm{H} & -2.50736707189100 \\ \mathrm{H} & -3.09815864485764 \\ \mathrm{C} & 2.63254700101297 \\ \mathrm{C} & 2.90084786240004 \\ \mathrm{C} & 2.57390473074227 \\ \mathrm{C} & 2.96335064851275 \\ \mathrm{C} & 2.73173019404354 \\ \mathrm{H} & 2.38274244412337 \\ \mathrm{C} & 2.89231183646685 \\ \mathrm{H} & 2.97666257262408 \\ \mathrm{H} & -2.49547029363409 \\ \mathrm{C} & -1.50919801741900 \\ \mathrm{C} & -0.36794564001434 \\ \mathrm{C} & -1.42954209145331 \\ \mathrm{C} & 0.87067427414228 \\ \mathrm{C} & -0.17452246612789 \\ \mathrm{C} & 0.99196723310779 \\ \mathrm{H} & 1.78566582233978 \\ \mathrm{C} & -0.84167781954475 \\ \mathrm{C} & -0.81295102269329 \\ \mathrm{C} & 0.37618543657189 \\ \mathrm{C} & 0.37775141550625 \\ \mathrm{H} & -1.76208263832985 \\ \mathrm{C} & 1.57753635788774 \\ \mathrm{C} & 1.56384749556820 \\ \mathrm{H} & 2.51363948527440 \\ \mathrm{O} & -2.69003848405355 \\ \mathrm{O} & -0.03928758225351 \\ & \end{array}$

$-0.78607707024534$

$-1.46562113305296$

$-0.75018736858113$

0.62746951591156

1.29217184384723

0.60907350961941

$-1.29814106560235$

2. 36282531814781

1.26668930186891

$-0.11385162030730$

1.98433096148994

$-0.75116038224644$

1. 38872263316924

3. 04964028448965

0.00332090519327

$-0.52494593213417$

4. 32379326155782

3.88744870911856

4.68658761501688

2.49872366976871

4.03053876794432

1.87861761818534

2.65119226799701

4.60916986894483

$-3.20944337965918$

$-3.73626543703966$

$-2.90570100501555$

$-3.95465601710217$

$-3.99510790644239$

$-3.03332027853102$

$-3.56714933939671$

$-3.65967906522987$

$-1.47989149491415$

0.53390642038173
0.70613064013088 $-0.51234437920845$ $-1.70354448381752$

$-1.74625876522110$

$-0.52190486437385$

0.69462949798776

$-2.63003614340594$

$-0.48263346212348$

0.79164561828506

0.73923697204830

$-0.40131037641333$

$-0.50872351411393$

$-1.65837354180509$

$-0.33570317331142$

$-1.68418434063958$

$-2.62341704370999$

1.93398869603051

2. 01289502954113

1. 93707151909545

2. 17576819347975

1.97657811996904

2.29989036145903

2.13803785136244

1.89256259877250

$-1.26033639828617$

$-2.55053427982234$

$-0.62059086332923$

$-3.25825969131641$

$-3.00926982231279$

$-1.33896830828988$

$-2.63391998648495$

$-3.14287082212094$

1.89534913109001

2.48865433999320 


\begin{tabular}{|c|c|}
\hline O & 3.04878680922207 \\
\hline O & 0.33311769136018 \\
\hline C & -2.16298053508006 \\
\hline $\mathrm{H}$ & -2.11324961249093 \\
\hline $\mathrm{H}$ & -2.94899765498385 \\
\hline S & -2.96914018299725 \\
\hline $\mathrm{C}$ & 2.35408457194808 \\
\hline $\mathrm{H}$ & 3.12262876957706 \\
\hline $\mathrm{H}$ & 2.41364139708706 \\
\hline S & 3.16842633208344 \\
\hline $\mathrm{C}$ & 2.75750930552102 \\
\hline $\mathrm{C}$ & 4.04158259235225 \\
\hline $\mathrm{H}$ & 4.08831306737795 \\
\hline $\mathrm{H}$ & 4.07512007429133 \\
\hline $\mathrm{H}$ & 4.93535295796726 \\
\hline $\mathrm{C}$ & 2.75884827953889 \\
\hline $\mathrm{H}$ & 3.64784046529989 \\
\hline $\mathrm{H}$ & 1.87535922834334 \\
\hline $\mathrm{H}$ & 2.75560311722930 \\
\hline $\mathrm{C}$ & 1.52522806165965 \\
\hline $\mathrm{H}$ & 0.59812778414757 \\
\hline $\mathrm{H}$ & 1.50716326441443 \\
\hline $\mathrm{H}$ & 1.53051267231664 \\
\hline $\mathrm{C}$ & 0.33667362874537 \\
\hline C & 1.73929378468482 \\
\hline $\mathrm{H}$ & 2.22866099917847 \\
\hline $\mathrm{H}$ & 2.38957892924324 \\
\hline $\mathrm{H}$ & 1.65722755544730 \\
\hline C & -0.52106725322860 \\
\hline $\mathrm{H}$ & -1.55066410798730 \\
\hline $\mathrm{H}$ & -0.10386778511565 \\
\hline $\mathrm{H}$ & -0.55375327306316 \\
\hline C & -0.28956024083605 \\
\hline $\mathrm{H}$ & -0.33057212183940 \\
\hline $\mathrm{H}$ & 0.30257903240153 \\
\hline $\mathrm{H}$ & -1.30733839269516 \\
\hline $\mathrm{C}$ & -3.10014462960741 \\
\hline $\mathrm{C}$ & -3.04630223295087 \\
\hline $\mathrm{H}$ & -3.15991461399759 \\
\hline $\mathrm{H}$ & -3.84963288359439 \\
\hline $\mathrm{H}$ & -2.09247316137334 \\
\hline $\mathrm{C}$ & -2.02899986782366 \\
\hline $\mathrm{H}$ & -1.02238552011354 \\
\hline $\mathrm{H}$ & -2.07893413707872 \\
\hline $\mathrm{H}$ & -2.17265473925312 \\
\hline $\mathrm{C}$ & -4.49721514216652 \\
\hline $\mathrm{H}$ & -5.28263426580387 \\
\hline $\mathrm{H}$ & -4.69042882308179 \\
\hline $\mathrm{H}$ & -4.57573117151651 \\
\hline $\mathrm{C}$ & -0.42800322718553 \\
\hline $\mathrm{C}$ & -1.87426934995479 \\
\hline $\mathrm{H}$ & -1.86638933368364 \\
\hline $\mathrm{H}$ & -2.44453925839592 \\
\hline $\mathrm{H}$ & -2.40803489860200 \\
\hline $\mathrm{C}$ & 0.31308659227319 \\
\hline $\mathrm{H}$ & -0.15171835813937 \\
\hline $\mathrm{H}$ & 1.36206183148723 \\
\hline & 30366486960 \\
\hline
\end{tabular}

$-0.83631299228881$

$-2.41722792204982$

$-2.94764619913263$

$-3.32905707433496$

$-3.50335927684071$

1. 55980854259937

1.97775465650763

2. 73723558841577

1. 25221657351876

$-2.54240735321682$

2. 25620397593525

3.11912126297946

3. 75198669544733

3. 77162057171036

2.48847624379116

1. 40771563796888

0.77467063949481

0.76600057670810

2.06618458966233

3.18681048196094

2. 60867300407911

3. 88220417056143

3. 78208911296142

$-4.57778790796893$

$-4.71472842363251$

$-3.74439883020193$

$-5.35498558463483$

$-5.16737472687924$

$-3.69610479289334$

$-3.60759218813225$

$-2.68805839143075$

$-4.12837044428797$

$-5.99056391582111$

$-6.45034656504235$

$-6.63861577136190$

$-5.95775368232040$

1. 34092629015459

2. 87406341394518

3. 33840814680721

3. 25631724897994

3. 20737868432104

0.91776635130415

1.13078862238510

$-0.14783007418715$

1. 46629912957202

0.94738410165870

1.24977289317135

1. 43257408819101

$-0.13367681707719$

6.21827191336813

6.74638806422232

7. 83642237502901

6.34718811899724

6.49789412447634

6.87745914413971

6.60063481106227

6.57414131386578

7. 96845655247409
1.90207420171067

0.65364210176439

$-0.55583597744176$

0.46132631837669

$-1.07751982707683$

2.22918775439893

2.11403531130630

2. 29340587038346

2. 92204612007097

$-0.64287236018857$

$-2.93035629852701$

$-2.91672641246700$

$-3.80970936372696$

$-2.04005291564246$

$-2.89902909241596$

$-4.21518577147880$

$-4.28698097150175$

$-4.27841415444649$

$-5.08890070721728$

$-2.97055514712278$

$-2.94423973769234$

$-2.12824527763759$

$-3.88942033277323$

$-4.66589002957539$

$-5.28726168506176$

$-5.40599367916404$

$-4.68503552395179$

$-6.27972064251640$

$-5.60357517347652$

$-5.24796340228604$

$-5.68023259232949$

$-6.60873492031525$

$-4.58001431185227$

$-5.57256621723032$

$-3.92780034872384$

$-4.18330194046036$

$-3.09291866850266$

$-2.95264975375119$

$-3.93611431286617$

$-2.31693109635792$

$-2.53447487540357$

$-4.12330401299879$

$-3.75568633502564$

$-4.35725366603979$

$-5.05915830293996$

$-3.63082205911518$

$-2.93253714513140$

$-4.59347282561920$

$-3.77480312102319$

1.78913511582967

1.77518448981330

1.68176734058185

0.93166647667984

2. 69727328094846

2. 97615762471696

3. 92690361638171

3.01755187914046

2.88648951416495 


\begin{tabular}{|c|c|}
\hline $\mathrm{C}$ & 0.25019687702626 \\
\hline $\mathrm{H}$ & 1.30031296718743 \\
\hline $\mathrm{H}$ & -0.25484728790649 \\
\hline $\mathrm{H}$ & 0.21155391730737 \\
\hline $\mathrm{C}$ & -0.44623946567543 \\
\hline $\mathrm{H}$ & -0.83931708498196 \\
\hline $\mathrm{H}$ & -1.26710118043100 \\
\hline $\mathrm{C}$ & 0.71584175287871 \\
\hline $\mathrm{C}$ & 0.29811593544471 \\
\hline $\mathrm{H}$ & 1.53344109501422 \\
\hline $\mathrm{H}$ & 1.08734145290683 \\
\hline $\mathrm{C}$ & -3.88769828765149 \\
\hline $\mathrm{H}$ & -3.56338507423069 \\
\hline $\mathrm{H}$ & -4.26653907056725 \\
\hline $\mathrm{C}$ & -4.98617990197473 \\
\hline $\mathrm{C}$ & -6.20038204713907 \\
\hline $\mathrm{H}$ & -4.57881392402423 \\
\hline $\mathrm{H}$ & -5.29058635959451 \\
\hline $\mathrm{C}$ & 0.87270777189685 \\
\hline $\mathrm{H}$ & 1.18369053464994 \\
\hline $\mathrm{H}$ & 1.76838050433329 \\
\hline $\mathrm{C}$ & -0.14848402295456 \\
\hline $\mathrm{C}$ & 0.42586397432243 \\
\hline $\mathrm{H}$ & -1.03370107509101 \\
\hline $\mathrm{H}$ & -0.46532384471691 \\
\hline $\mathrm{C}$ & 4.31585287569903 \\
\hline $\mathrm{H}$ & 4.61092197473328 \\
\hline $\mathrm{H}$ & 4.12258087121976 \\
\hline C & 5.43309292004151 \\
\hline $\mathrm{C}$ & 6.73872659395255 \\
\hline $\mathrm{H}$ & 5.11343583589378 \\
\hline $\mathrm{H}$ & 5.59552899920875 \\
\hline $\mathrm{H}$ & -6.62489992727192 \\
\hline $\mathrm{H}$ & -6.98958387806429 \\
\hline $\mathrm{H}$ & -5.93314177863634 \\
\hline $\mathrm{H}$ & 7.53185315050651 \\
\hline $\mathrm{H}$ & 6.61487925844330 \\
\hline $\mathrm{H}$ & 7.08665563193406 \\
\hline $\mathrm{H}$ & -0.07888950467141 \\
\hline $\mathrm{H}$ & -0.49274231095712 \\
\hline $\mathrm{H}$ & 1.14251917900032 \\
\hline $\mathrm{H}$ & 1.31293676508925 \\
\hline $\mathrm{H}$ & -0.30621697913759 \\
\hline & \\
\hline
\end{tabular}

6.64351186454259

6.34322850834011

6.19205481315274

7.73168096625136

$-0.01065699470054$

$-1.00095919247516$

0.58192804515663

$-0.08834732200614$

$-0.75815434312391$

$-0.64433119931714$

0.92191148132019

$-2.20791649356151$

$-2.96775722605766$

$-2.72524035965816$

$-1.34440650143345$

$-2.18669590934552$

$-0.82050110299529$

$-0.57789586674829$

$-3.24653023083441$

$-2.54295589715154$

$-3.76062441004576$

$-4.24307651733411$

$-5.07145574914619$

$-3.69192707316429$

$-4.91282475521822$

$-0.70010976019825$

0.35649827917808

$-1.02062085882807$

$-1.54060300090285$

$-1.40110283209232$

$-2.58615110933544$

$-1.22987305954457$

$-2.71860422615340$

$-1.55895556385971$

$-2.93374351457161$

$-2.00721914538309$

$-1.72824862471270$

$-0.36317629270236$

$-1.77132151873865$

$-0.19229430058583$

$-0.83292786787047$

$-5.63190208851968$

$-5.79385900646481$

$-4.43440679900411$
0.46522131892318

0.43193147492337

$-0.39332697831924$

0.34962585338057

3.76487949906699

3.53573351946960

4.17531052162213

4.74992364539286

6.06438341742962

4. 28181077868458

4.95564903869665

2.22668004559392

2. 94359323224507

1.33642517329351

2.83934141675786

3.24634676803498

3.70850486095335

2.11950514011805

1. 70684029456981

2.47812940601370

1. 35016152635866

2.24638885219799

3. 40096420849175

2.57886630997082

1.43901148373017

2.57392939957490

2. 60141430541799

3. 60159836385942

1. 96209082646706

2. 75372437229660

1.93409100853929

0.92516549884573

2.38859541954078

3.66742191745669

4.00102834666428

2.30937101783424

3. 79117485179825

2.77311302233953

5.89211132758100

6.56695277079470

6.75497587082091

3. 08794171884961

3. 77176002434586

4. 24191879304091

\subsection{Compound 4Et}

Final single point energy: -3042.557992533471

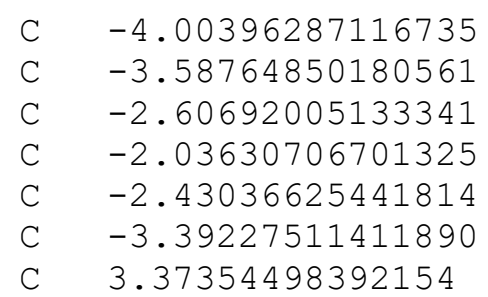

0.37353974977287

$-0.69706326327864$

$-0.57072449898043$

0.69580640854766

1.78464002890688

1.60804074067611

0.69865637062896
2.07136543584040
1.27105508723906
0.28527677117559
0.05878451549532
0.85093843051956
1.85215303684679
0.09440112996529 
$-0.62046312512580$

0.90373737888038

$-1.66617820687671$

$-0.13198078173097$

1.92008461220377

$-1.41417433910898$

$-2.25727821513523$

4.97139199935942

4.42825597285259

4. 63592851458140

3.54412014485902

3. 82621525966612

2. 83225235301043

2.88182879300002

3. 91594178750227

$-3.46458697186946$

$-2.68198363623559$

$-4.29462285245733$

$-2.75789768381687$

$-4.23406305948126$

$-3.45722550457942$

2. 05656777233316

$-0.88212700830380$

$-1.78282062808380$

$-2.37577287269780$

$-1.44951978274964$

3. 49044302473880

1. 90083717932372

2. 42527425687205

1.53636551752117

$-3.39215171917318$

0.84236590896646

$-1.66986684708421$

2.46770876525266

$-4.76643002237239$

$-3.42504562578990$

$-2.09842590443493$

0.16274940063706

1. 44999453706002

1. 24769545683844

1.81992742273002

2. 24871815217148

$-0.29824515051461$

0.45599151765929

$-1.23295505917535$

$-0.46027152185181$

$-0.91876330051887$

$-1.87911041054687$

$-0.61909827109821$

$-1.07447749515155$

$-5.22223305348244$

$-6.68457803738620$

$-6.82000284029322$

$-7.36473964981818$

$-6.98335813141222$

$-4.84658821822722$

$-4.93089593877747$

$-3.81873245216165$
0.52572500699817

$-1.20171211071494$

$-0.41367447569044$

$-2.11409527271454$

$-1.50204111181148$

$-1.70048677147981$

$-2.37059261183861$

$-1.31352164244797$

$-0.78916133605286$

$-1.10793954641306$

0.21745482674771

$-0.46177947063697$

0.93233470207695

0.51307150388692

$-0.71461288834798$

1.21693696437405

0.10610725512673

1.86445079744532

$-0.40064814548281$

1. 42719629906898

0.33470791347141

2.00292109480108

1.85788065216860

$-0.54870472786891$

$-0.74514711030089$

$-1.50496771277004$

0.58642821218229

1.02737805897845

1.11226447559885

2.02062758481599

$-0.01786634842431$

$-0.90556700470486$

1.40817049479365

2. 45101719126388

1.96424250338473

1.56532688075478

$-1.56624074745942$

3.14120915406426

3. 93092162707981

4. 68005559133090

4.45857486485225

3. 28246157017450

2.45760314286839

1. 74942873702720

1. 90848466356804

3. 20516803281870

4.14583891638254

3.65627783977796

4.64111075538799

4. 91653668236195

3.03276940863496

2. 64006992500674

2. 41621121294225

3.45759111399795

1. 75466192483483

4. 29589541701294

4. 13182252450340

4.60328007651217 


\begin{tabular}{|c|c|}
\hline $\mathrm{H}$ & -0.41686743114580 \\
\hline $\mathrm{C}$ & -2.45900629745710 \\
\hline $\mathrm{H}$ & -2.74547705885162 \\
\hline $\mathrm{H}$ & -3.09062430538587 \\
\hline $\mathrm{H}$ & -2.68335875982516 \\
\hline $\mathrm{C}$ & 4.67398139391130 \\
\hline $\mathrm{C}$ & 3.77671230189207 \\
\hline $\mathrm{H}$ & 2.77358701829172 \\
\hline $\mathrm{H}$ & 3.67399079194878 \\
\hline $\mathrm{H}$ & 4.20448791916350 \\
\hline $\mathrm{C}$ & 4.82253912050359 \\
\hline $\mathrm{H}$ & 5.47429525475387 \\
\hline $\mathrm{H}$ & 3.85667899676196 \\
\hline $\mathrm{H}$ & 5.26571915995679 \\
\hline $\mathrm{C}$ & 6.08042793662868 \\
\hline $\mathrm{H}$ & 6.03978301130130 \\
\hline $\mathrm{H}$ & 6.73637868563005 \\
\hline $\mathrm{H}$ & 6.53707458328810 \\
\hline $\mathrm{C}$ & 1.94588781997489 \\
\hline $\mathrm{C}$ & 1.49515241899878 \\
\hline $\mathrm{H}$ & 1.75954450347871 \\
\hline $\mathrm{H}$ & 0.41456035576287 \\
\hline $\mathrm{H}$ & 1.98050029196523 \\
\hline $\mathrm{C}$ & 1.24906560748927 \\
\hline $\mathrm{H}$ & 1.52622536925275 \\
\hline $\mathrm{H}$ & 1.54528948479091 \\
\hline $\mathrm{H}$ & 0.16049030197130 \\
\hline $\mathrm{C}$ & 3.46465993555789 \\
\hline $\mathrm{H}$ & 4.02076539044818 \\
\hline $\mathrm{H}$ & 3.84577742178407 \\
\hline $\mathrm{H}$ & 3.68820155392121 \\
\hline $\mathrm{C}$ & -1.53958379581209 \\
\hline $\mathrm{H}$ & -2.30322347506382 \\
\hline $\mathrm{H}$ & -2.00466258505474 \\
\hline $\mathrm{C}$ & -0.34361002668496 \\
\hline $\mathrm{H}$ & 0.12481607225556 \\
\hline $\mathrm{H}$ & -0.65572671685921 \\
\hline $\mathrm{H}$ & 0.39869717429656 \\
\hline $\mathrm{C}$ & 1.62217703666735 \\
\hline $\mathrm{H}$ & 1.09661173870800 \\
\hline $\mathrm{H}$ & 1.05544697158387 \\
\hline $\mathrm{C}$ & 1.81637503037370 \\
\hline $\mathrm{H}$ & 2.39278538508683 \\
\hline $\mathrm{H}$ & 0.84559919767001 \\
\hline $\mathrm{H}$ & 2.35332275397366 \\
\hline $\mathrm{C}$ & 0.75476146941957 \\
\hline $\mathrm{H}$ & 0.79747178777130 \\
\hline $\mathrm{H}$ & 1.75336433949126 \\
\hline $\mathrm{C}$ & -0.26433444678354 \\
\hline $\mathrm{H}$ & -0.30997858365545 \\
\hline $\mathrm{H}$ & -1.26016829762109 \\
\hline $\mathrm{H}$ & 0.01764512873876 \\
\hline $\mathrm{C}$ & 0.75094149310093 \\
\hline $\mathrm{H}$ & 1.23355574231956 \\
\hline $\mathrm{H}$ & 1.47529509083051 \\
\hline $\mathrm{C}$ & -0.49397515713896 \\
\hline $\mathrm{H}$ & -1.20600246220829 \\
\hline $\mathrm{H}$ & -0.21175961623911 \\
\hline
\end{tabular}

$-5.51042738432914$

$-5.13541799295500$

$-4.12846383520413$

$-5.42367512600392$

$-5.81663159173032$

0.16323179449743

1.15905873072596

0.74967205030393

2.10664774410074

1. 37682329117486

$-1.10858300286873$

$-1.84557374288745$

$-1.58196762047489$

$-0.85201297002818$

0.78125968668718

1. 72057761812714

0.09758614203288

0.98900235684829

5.70598165523752

7.09502476460033

7.87235791437267

7.13546382521216

7.33634980725359

5.40863518834487

6.16193138323038

4. 42834036777700

5.41699185250263

5.75839392376397

5.99282109358096

4.81543505928305

6.53994590040408

1. 27060861734693

0.56832768876059

2. 25885094208554

1. 30149604591785

0.31627466589340

1. 59549548058367

2. 01682766609921

$-0.90462991831897$

0.01963369655414

$-1.73838331035518$

$-1.07666103530968$

$-0.24893623891410$

$-1.11571757104367$

$-2.00461581397118$

2.58567970094306

1. 71030040482266

3. 03455434323956

3. 58819138938275

4. 47852103122366

3. 14271057770144

3.89762933588913

$-2.94408063465366$

$-2.27745365175626$

$-3.70949555023676$

$-3.57913355105219$

$-2.81811719882733$

$-4.18184689279762$
5.12553649130890

3.38040882604188

3.69707985825515

2. 53480335566729

4. 20601711750670

$-3.49765607592454$

$-4.26789958131679$

$-4.41402151994361$

$-3.73384026445365$

$-5.25178865711956$

$-4.35313972036095$

$-3.87566107463990$

$-4.55004253375087$

$-5.31941216182553$

$-3.31175044154511$

$-2.75402354468118$

$-2.76549682567525$

$-4.28452429593058$

$-2.14653617853422$

$-1.63454217128231$

$-2.35883980864448$

$-1.47755284041644$

$-0.68453497883274$

$-3.49471816868978$

$-4.23920806309494$

$-3.87825731688693$

$-3.40172600232893$

$-2.39596049860763$

$-1.48355230005762$

$-2.79860681105099$

$-3.12782712425315$

$-2.19941063725614$

$-2.55869670270734$

$-2.11243727301220$

$-3.13003062823476$

$-3.17348099379614$

$-4.13657873348756$

$-2.77117162671032$

2. 44537654748674

2.20492807168247

2.03569970415572

3. 94023529536922

4.36243835714883

4.44280894556761

4.15257063722734

3. 30768017606662

3. 95833546586209

3.27367902565613

3. 82767193778949

3.19848107770949

3. 86521808542859

4.83940680769623

$-2.69892590654096$

$-3.41598937109037$

$-2.40660702119485$

$-3.30521491421587$

$-3.63330015311412$

$-4.17436317068125$ 


\subsection{Compound 4Pr}

Final single point energy: -3199.735880745184

\begin{tabular}{|c|c|}
\hline $\mathrm{C}$ & -3.96509671700382 \\
\hline $\mathrm{C}$ & -3.55192420119688 \\
\hline $\mathrm{C}$ & -2.60200946079457 \\
\hline $\mathrm{C}$ & -2.06108335426819 \\
\hline $\mathrm{C}$ & -2.44750598962797 \\
\hline $\mathrm{C}$ & -3.37898546336325 \\
\hline $\mathrm{C}$ & 3.34124249189257 \\
\hline $\mathrm{C}$ & 3.10640192093207 \\
\hline $\mathrm{C}$ & 3.81855000452393 \\
\hline $\mathrm{C}$ & 3.22622397142243 \\
\hline $\mathrm{C}$ & 4.05638898851155 \\
\hline $\mathrm{H}$ & 4.02738204717860 \\
\hline $\mathrm{C}$ & 3.71520027136913 \\
\hline $\mathrm{H}$ & 3.82575731202591 \\
\hline $\mathrm{H}$ & -0.55948008194303 \\
\hline $\mathrm{C}$ & 0.20616266471127 \\
\hline $\mathrm{C}$ & 1.56087014068908 \\
\hline $\mathrm{C}$ & -0.20306852842064 \\
\hline $\mathrm{C}$ & 2.48086401973187 \\
\hline $\mathrm{C}$ & 0.75722731238219 \\
\hline $\mathrm{C}$ & 2.10445979639250 \\
\hline $\mathrm{H}$ & 3.53449210272397 \\
\hline $\mathrm{C}$ & -1.51090099774261 \\
\hline $\mathrm{C}$ & -1.15905247121706 \\
\hline $\mathrm{C}$ & -0.59701894318593 \\
\hline $\mathrm{C}$ & 0.15600389881042 \\
\hline $\mathrm{C}$ & 0.73952606828353 \\
\hline $\mathrm{C}$ & 1.13297676397438 \\
\hline O & 0.35351714543758 \\
\hline O & 2.92546798133182 \\
\hline $\mathrm{C}$ & -2.19757931566051 \\
\hline $\mathrm{H}$ & -3.09857091318610 \\
\hline $\mathrm{H}$ & -1.80078390267094 \\
\hline S & -1.96625552313761 \\
\hline $\mathrm{C}$ & 3.14181857976095 \\
\hline $\mathrm{H}$ & 4.09875947723807 \\
\hline $\mathrm{H}$ & 2.87241096655147 \\
\hline S & 2.90105150346879 \\
\hline O & -1.13425001927426 \\
\hline $\mathrm{H}$ & -3.99029275930924 \\
\hline $\mathrm{H}$ & -3.64270838711413 \\
\hline $\mathrm{H}$ & 1.51183926277981 \\
\hline $\mathrm{H}$ & -2.54194330565056 \\
\hline O & 0.47432231459256 \\
\hline $\mathrm{C}$ & -5.01855032050445 \\
\hline $\mathrm{C}$ & -5.33433105041430 \\
\hline $\mathrm{H}$ & -6.08015858121263 \\
\hline $\mathrm{H}$ & -4.44804946745066 \\
\hline
\end{tabular}

0.36449437270105 $-0.71004676324040$

$-0.58274288392458$

0.68995739395779

1.78201787603548

1.60531191492970

0.70717665490104

$-0.61522220823926$

0.91614415494317

$-1.65923878097547$

$-0.11672339632407$

1. 93480727489859

$-1.40371954138636$

$-2.24597767939423$

4. 95071335507753

4.41743759202348

4.62727998595462

3.54664805310008

3.82585290095736

2.85107657717682

2.89189660011146

3. 91519729767969

$-3.49686643410863$

$-2.69987433751385$

$-4.32001833267619$

$-2.76034906196576$

$-4.23750950414962$

$-3.45278528946405$

2. 10740429696404

$-0.89772775780336$

$-1.80077563257384$

$-2.39110981497228$

$-1.47096429846314$

3. 48718226981891

1. 91037852503746

2.43246437654252

1. 54889806895385

$-3.38465553949828$

0.84414571385424

$-1.68781423602459$

2.46903009724630

$-4.76756195680170$

$-3.47661169706639$

$-2.09130558241297$

0.15058510943739

1. 44845878534692

1. 24449729857215

1.85376691820111
2.08406489281250

1.28803790318679

0.27230474365530

0.01135960326716

0.80333321618002

1.83197606693048

0.14963613871254

0.57778730179775

$-1.14530328814678$

$-0.36131445740834$

$-2.05562599994139$

$-1.44706029463621$

$-1.64425001064892$

$-2.31303463342006$

$-1.38245072884548$

$-0.83077741186413$

$-1.11519693433337$

0.17813135216599

$-0.43756383155351$

0.93082403706939

0.53918022242998

$-0.66468139548966$

1. 20495946475931

0.10803248957073

1.86426002080194

$-0.37700467423096$

1. 45496254959394

0.37200477027640

2. 01660619365316

1.90528666770503

$-0.55553999423856$

$-0.75279219713094$

$-1.51094239619779$

0.49797750047697

1.07972916767112

1.18481035632666

2. 06770077648848

0.03697810626123

$-0.98891557265085$

1. 45254779631531

2. 42673489275073

2. 00070899813554

1.53078473227908

$-1.53355309175109$

3. 18755765586561

3. 95387920717793

4. 72708013817567

4. 45029622666302 


\begin{tabular}{|c|c|}
\hline $\mathrm{H}$ & -5.74235044410337 \\
\hline $\mathrm{C}$ & -6.33243671293631 \\
\hline $\mathrm{H}$ & -6.73020905129759 \\
\hline $\mathrm{H}$ & -6.18446975762860 \\
\hline $\mathrm{H}$ & -7.08886040748355 \\
\hline C & -4.49928436532865 \\
\hline $\mathrm{H}$ & -4.31029304329393 \\
\hline $\mathrm{H}$ & -3.56547714006552 \\
\hline $\mathrm{H}$ & -5.23423179282959 \\
\hline C & -0.99361321798511 \\
\hline C & -0.66268152021979 \\
\hline $\mathrm{H}$ & 0.40263451791686 \\
\hline $\mathrm{H}$ & -0.93472062252344 \\
\hline $\mathrm{H}$ & -1.21449570957075 \\
\hline C & -0.20763780372177 \\
\hline $\mathrm{H}$ & 0.87196346715948 \\
\hline $\mathrm{H}$ & -0.42734578057380 \\
\hline $\mathrm{H}$ & -0.48405049197234 \\
\hline C & -2.49668496601912 \\
\hline $\mathrm{H}$ & -2.79214175658406 \\
\hline $\mathrm{H}$ & -3.11427238128318 \\
\hline $\mathrm{H}$ & -2.73041490128711 \\
\hline C & 4.66408411658727 \\
\hline C & 3.74191677551908 \\
\hline $\mathrm{H}$ & 2.75214841553808 \\
\hline $\mathrm{H}$ & 3.60737137473140 \\
\hline $\mathrm{H}$ & 4.16830277619861 \\
\hline $\mathrm{C}$ & 4.85210165073891 \\
\hline $\mathrm{H}$ & 5.52133052718384 \\
\hline $\mathrm{H}$ & 3.90088796806689 \\
\hline $\mathrm{H}$ & 5.29301743094522 \\
\hline C & 6.05166978733861 \\
\hline $\mathrm{H}$ & 5.98248192684212 \\
\hline $\mathrm{H}$ & 6.72381468393030 \\
\hline $\mathrm{H}$ & 6.50699752384134 \\
\hline $\mathrm{C}$ & 1.97240572062265 \\
\hline $\mathrm{C}$ & 1.51556658613363 \\
\hline $\mathrm{H}$ & 1.79722759945835 \\
\hline $\mathrm{H}$ & 0.43204858704155 \\
\hline $\mathrm{H}$ & 1.98207189042903 \\
\hline $\mathrm{C}$ & 1.30224777601217 \\
\hline $\mathrm{H}$ & 1.59579690599304 \\
\hline $\mathrm{H}$ & 1.60387073022530 \\
\hline $\mathrm{H}$ & 0.21205318674050 \\
\hline C & 3.49613370370519 \\
\hline $\mathrm{H}$ & 4.03347087874768 \\
\hline $\mathrm{H}$ & 3.88396238370461 \\
\hline $\mathrm{H}$ & 3.73673989121148 \\
\hline C & -1.66105867217284 \\
\hline $\mathrm{H}$ & -2.41608473117645 \\
\hline $\mathrm{H}$ & -2.15166860984244 \\
\hline $\mathrm{C}$ & -0.50562743126419 \\
\hline $\mathrm{H}$ & -0.00603013257691 \\
\hline C & -0.96893996922497 \\
\hline $\mathrm{H}$ & 0.22544071333294 \\
\hline C & 1.60810264232125 \\
\hline $\mathrm{H}$ & 1.19205056391799 \\
\hline $\mathrm{H}$ & 0.94528044842836 \\
\hline
\end{tabular}

2. 22130874128371

$-0.36387406222248$

0.36237317798876

$-1.30724015434708$

$-0.53113991043425$

$-0.89188402971609$

$-1.85952837608905$

$-0.55449582028772$

$-1.04665682622418$

$-5.27075979793213$

$-6.72381904312191$

$-6.85038975463789$

$-7.42171378699726$

$-7.00633285001941$

$-4.91828761836014$

$-4.99704738701445$

$-3.89736034505215$

$-5.59924261586252$

$-5.19660584752315$

$-4.19650226211572$

$-5.47274594911948$

$-5.89315059670278$

0.18776639791884

1.15382691750948

0.71402735149658

2.09939753282077

1.38079338966654

$-1.08430921976410$

$-1.80006083055657$

$-1.58563121313238$

$-0.82150296175643$

0.84634661984753

1.78840492561095

0.18534173814534

1. 05975200227021

5.69174582316083

7.08361045893015

7.85862799840190

7.12717384636309

7.32537403749276

5.39396501032525

6.14550525243350

4. 41269419576805

5.40525383959171

5.73893888770140

5.97922332950360

4.79227152827798

6.51469453434553

1. 24892349894052

0.52202610675736

2. 22496403097247

1. 31214699786251

0.33895920012361

1.69165066457912

2.03673001529395

$-0.88765410669942$

0.11957139557931

$-1.54989064172014$
3. 29622080074701

2. 55312551216758

1. 83832271028236

2. 02202677033181

3. 32681528820872

4.20523544732486

3.73486627707665

4.66406667827740

5.00176224318753

3.00938598184839

2.59256058318319

2. 38458936714241

3.39117904156788

1.69136157532721

4.29292114459720

4. 14592156672974

4.61675535023558

5.10436160716498

3.33528127889629

3. 66520370457240

2. 47541906628450

4.14539722702409

$-3.43764125939565$

$-4.21658165008874$

$-4.36634056737998$

$-3.68602187814027$

$-5.19899279083647$

$-4.28488418149218$

$-3.79923981434048$

$-4.48361991339423$

$-5.25054473444646$

$-3.25004330218597$

$-2.69987234301862$

$-2.69571142178796$

$-4.22221324682291$

$-2.14991421033718$

$-1.65058722343875$

$-2.37083478867665$

$-1.51588748122011$

$-0.69129265447603$

$-3.51136860693389$

$-4.25128940010443$

$-3.88797729894769$

$-3.44065868865286$

$-2.36825344449389$

$-1.44616353799017$

$-2.75568160683467$

$-3.10080590403600$

$-2.26586001329994$

$-2.59619567564014$

$-2.16914166088512$

$-3.25116794909485$

$-3.25692067011358$

$-4.66095291053720$

$-2.88089120525630$

2.50504939381200

2.45918956108499

1. 94883973591512 


$\begin{array}{ll}\mathrm{C} & 1.76284389196229 \\ \mathrm{C} & 2.71639416494456 \\ \mathrm{H} & 0.76257537732391 \\ \mathrm{H} & 2.10805306656493 \\ \mathrm{C} & 0.64367744954998 \\ \mathrm{H} & 0.51548137517520 \\ \mathrm{H} & 1.69458527906613 \\ \mathrm{C} & -0.26197887141357 \\ \mathrm{H} & -0.15899606454016 \\ \mathrm{H} & -1.30293556842735 \\ \mathrm{C} & 0.07618798532879 \\ \mathrm{C} & 0.79363205648502 \\ \mathrm{H} & 1.29844724804679 \\ \mathrm{H} & 1.50656486733059 \\ \mathrm{C} & -0.43547991831591 \\ \mathrm{H} & -1.12584325214250 \\ \mathrm{C} & -0.05394765745942 \\ \mathrm{H} & -0.96212235537263 \\ \mathrm{H} & 3.71656436533572 \\ \mathrm{H} & 2.79413669646658 \\ \mathrm{H} & 2.37327836444099 \\ \mathrm{H} & 1.11382965111329 \\ \mathrm{H} & -0.06163885466033 \\ \mathrm{H} & -0.56608075056625 \\ \mathrm{H} & -0.12340574216680 \\ \mathrm{H} & -1.45277113158913 \\ \mathrm{H} & -1.68616900953160 \\ \mathrm{H} & 0.47314046283428 \\ \mathrm{H} & -0.94141318558910 \\ \mathrm{H} & 0.59953106984023 \\ & -1.536\end{array}$

$-1.37915388853366$

$-0.54208274236263$

$-1.39183707304706$

$-2.41740608287951$

2.68913188325563

1.86435414879426

3. 00272849683321

3.85419356408229

4. 65761758069417

3. 52317097952455

4. 38195003155156

$-2.92401534031231$

$-2.25337203565738$

$-3.69893242881013$

$-3.55027740475139$

$-2.75543402599205$

$-4.40386303850311$

$-4.16841672174176$

$-0.52796942262978$

$-0.95229469033421$

0.49374116155867

4.72584332658711

3. 60980688164428

5. 22575832891725

1.73378397717609

2.67372928026087

0.96562086008940

$-3.81165675691188$

$-4.82940236872117$

$-5.23362265477935$
3.93724859645826

4.79602128556178

4. 38694164907002

3. 91200131097900

3.30676767739869

4. 01213292238072

3.33934617926615

3. 69125224957164

2. 95601959568639

3. 65289628598083

5.09037420501915

$-2.66671047012532$

$-3.36593514808314$

$-2.36764077698150$

$-3.32173592713303$

$-3.62247811926896$

$-4.53662988535063$

$-2.58693143379578$

4. 35867184539833

5.80690126700260

4.88354734155738

5.14950358723098

5.85455639943702

5.35416032708441

$-5.35220706943972$

$-4.67310831015481$

$-5.05795376586401$

$-5.29193101553221$

$-5.01154114124828$

$-4.25065181746419$

\subsection{Compound 5Et}

Final single point energy: -3042.564367026722

$\begin{array}{ll}\text { C } & -1.96674960614231 \\ \text { C } & -1.98631988811052 \\ \text { C } & -2.10414623580161 \\ \text { C } & -2.21464622769012 \\ \text { C } & -2.09021670691854 \\ \text { C } & -1.98573446493138 \\ \text { C } & 3.62010682282186 \\ \text { C } & 3.79441734809492 \\ \text { C } & 3.35686672725476 \\ \text { C } & 3.60055064630100 \\ \text { C } & 3.27670292726120 \\ \text { C } & 3.33380368242213 \\ \text { H } & -1.62649113191060 \\ \text { C } & -0.64193163072244 \\ \text { C } & 0.50096442220645 \\ \text { C } & -0.56288167483176 \\ \text { C } & 1.72296901948996 \\ \text { C } & 0.69566767521907 \\ \text { C } & 1.84664304211431 \\ \text { H } & 2.62768230896061 \\ \text { C } & -0.93711085933778\end{array}$

$-2.06241526176748$

$-2.64278637028807$

$-1.89116883455467$

$-0.49838426891653$

0.11757980462807

$-0.66519156552569$

$-0.54944406354068$

$-1.93301445316276$

0.23392146355242

$-2.54052999947997$

$-0.39213341124351$

$-1.79518270515589$

3. 65027581600752

3. 31966028087884

3.80314504048976

2. 41139714873444

3. 25292924017639

1. 96834776757282

2. 32657861667411

3. 53707256760663

$-2.28010467987401$
3.51442823596905

2.24498477944999

1.07049765898372

1.17031490075232

2. 42795133554987

3. 57433283870936

2.86530681981218

2.80209194569909

1.73633174058805

1.55577501327759

0.48653766826612

0.40920893323230

0.47077946241002

0.77682159001770

0.13138358665795

1.83047520829149

0.51827137810075

2.28069328621198

1.56012719157595

$-0.00234942345040$

$-1.18381874950486$ 


\begin{tabular}{|c|c|}
\hline $\mathrm{C}$ & -1.11982898849826 \\
\hline C & 0.35303368351460 \\
\hline $\mathrm{C}$ & -0.06588500035711 \\
\hline $\mathrm{H}$ & -2.13027232673737 \\
\hline $\mathrm{C}$ & 1.44726821994472 \\
\hline C & 1.22541696228594 \\
\hline $\mathrm{H}$ & 2.09035839747385 \\
\hline O & 0.78009693175186 \\
\hline O & 0.52367022905460 \\
\hline $\mathrm{C}$ & -2.12747760976178 \\
\hline $\mathrm{H}$ & -2.19047507009662 \\
\hline $\mathrm{H}$ & -3.03596916978780 \\
\hline S & -2.10379518998453 \\
\hline $\mathrm{C}$ & 3.22287915326861 \\
\hline $\mathrm{H}$ & 3.93483447697156 \\
\hline $\mathrm{H}$ & 3.53807844380086 \\
\hline S & 3.14586051707909 \\
\hline O & -2.42937898663642 \\
\hline $\mathrm{H}$ & -1.91372845846259 \\
\hline $\mathrm{H}$ & -1.90705360529738 \\
\hline $\mathrm{H}$ & 3.70865397720762 \\
\hline $\mathrm{H}$ & 3.66116680973887 \\
\hline O & 3.14958836172520 \\
\hline $\mathrm{C}$ & 0.37106049388464 \\
\hline C & 1.73502086319470 \\
\hline $\mathrm{H}$ & 1.59507697791915 \\
\hline $\mathrm{H}$ & 2.41520935204277 \\
\hline $\mathrm{H}$ & 2.22024941825878 \\
\hline C & -0.52460571469044 \\
\hline $\mathrm{H}$ & -0.10418308866116 \\
\hline $\mathrm{H}$ & -1.53360829066670 \\
\hline $\mathrm{H}$ & -0.61067244698923 \\
\hline $\mathrm{C}$ & -0.26757751353189 \\
\hline $\mathrm{H}$ & 0.35275206688357 \\
\hline $\mathrm{H}$ & -0.37519856766529 \\
\hline $\mathrm{H}$ & -1.25892551835500 \\
\hline C & -0.27078542724150 \\
\hline $\mathrm{C}$ & 0.46532048572112 \\
\hline $\mathrm{H}$ & 1.54051875181539 \\
\hline $\mathrm{H}$ & 0.31710211335236 \\
\hline $\mathrm{H}$ & 0.09130419837276 \\
\hline $\mathrm{C}$ & -1.75756653421504 \\
\hline $\mathrm{H}$ & -1.85097408794108 \\
\hline $\mathrm{H}$ & -2.33310965320803 \\
\hline $\mathrm{H}$ & -2.21529118218758 \\
\hline $\mathrm{C}$ & 0.29968500034456 \\
\hline $\mathrm{H}$ & -0.20619065247717 \\
\hline $\mathrm{H}$ & 0.15958813134466 \\
\hline $\mathrm{H}$ & 1.36860663627988 \\
\hline $\mathrm{C}$ & 4.20621589117674 \\
\hline $\mathrm{C}$ & 4.27542460386696 \\
\hline $\mathrm{H}$ & 5.01459413666638 \\
\hline $\mathrm{H}$ & 3.30792947203783 \\
\hline $\mathrm{H}$ & 4.56906269500729 \\
\hline $\mathrm{C}$ & 3.20024150158196 \\
\hline $\mathrm{H}$ & 2.19338293777438 \\
\hline $\mathrm{H}$ & 3.15693540770183 \\
\hline $\mathrm{H}$ & 3.49102785474931 \\
\hline
\end{tabular}

$-1.58359789995222$

$-2.70209436246331$

$-1.27036213414305$

$-1.29454109943243$

$-2.28650078761445$

$-1.59111574407846$

$-1.30015859696206$

1. 14293535535168

$-3.51941047676449$

$-2.58597646669014$

$-3.66374208483354$

$-2.30706761340786$

1.90926819703201

1.74500413692729

2. 23001516590237

2.01801384190832

$-2.67137531013714$

0.24704081684646

$-3.71744520422900$

$-0.15596375052902$

$-0.03932023292534$

$-3.61685269375520$

0.37902021609057

4.87989543234542

5.27263774958044

6.05601699855262

5.66280323519632

4. 42633017697781

4.36260751007336

3. 45440421353615

4. 12922565449784

5. 11719311994641

6.14871538699700

6.54341649345142

6.92941745552069

5. 94171684792276

$-0.59131523319055$

0.76666799353191

0.64577286965492

1. 26126320071078

1. 42811041648242

$-0.33915473680381$

0.12159288358733

$-1.26875700864736$

0.34019321166153

$-1.50597867177309$

$-2.47532896781772$

$-1.04454477700313$

$-1.68824309933778$

$-2.78151597745647$

$-1.95004909494655$

$-1.14715908243797$

$-1.50590623606908$

$-2.59404883754368$

$-3.93247849725387$

$-3.54546123549820$

$-4.60387552239663$

$-4.53058620946695$
$-2.38302484623015$

$-0.84087485349994$

$-3.24267410204636$

$-2.63891511961167$

$-1.62008506528761$

$-2.80677332173529$

$-3.38967541837245$

3. 37870197337134

0.25554179037083

$-0.28531639652225$

$-0.10929021667534$

$-0.82688412665331$

2.61772900748790

1.86859323347866

1.19541194532855

2.87924524973175

$-1.15595752661731$

0.03248919712649

2.14102078871915

4.52601963146676

3.81541310989102

1.45220590838591

$-0.64735789698571$

$-0.96248030537226$

$-1.56000431612764$

$-2.31036071684208$

$-0.79730209772375$

$-2.05395517419866$

$-2.11059830699356$

$-2.54998783182782$

$-1.76410261548768$

$-2.89973307385483$

$-0.34780543208517$

0.46220626007444

$-1.10809047934669$

0.06167443540496

$-4.61025613094188$

$-4.64009504287756$

$-4.49128788827372$

$-5.60622300717660$

$-3.85446775644377$

$-4.92129995515809$

$-5.90907895066996$

$-4.93380290596059$

$-4.19757329415887$

$-5.72006843513137$

$-5.72344262939160$

$-6.70327530153394$

$-5.58584591351050$

4.02074558204376

5.31531186060223

5.24394039078098

5.56611012289155

6.14914905723367

4. 24144777016960

4. 41360520323937

3.38101701369742

5.11147617545359 


\begin{tabular}{|c|c|}
\hline $\mathrm{C}$ & 5.60928012541078 \\
\hline $\mathrm{H}$ & 6.34692587554514 \\
\hline $\mathrm{H}$ & 5.93466346271109 \\
\hline $\mathrm{H}$ & 5.61155241687580 \\
\hline $\mathrm{C}$ & -1.91753665468581 \\
\hline C & -1.76580368073627 \\
\hline $\mathrm{H}$ & -1.74429349638047 \\
\hline $\mathrm{H}$ & -2.60101723000132 \\
\hline $\mathrm{H}$ & -0.83653675203014 \\
\hline$C$ & -3.23242505240284 \\
\hline $\mathrm{H}$ & -3.21824859538409 \\
\hline $\mathrm{H}$ & -3.38223174788541 \\
\hline$H$ & -4.09550517398206 \\
\hline$C$ & -0.72320537853574 \\
\hline H & -0.82323408853865 \\
\hline$H$ & -0.65461691248369 \\
\hline $\mathrm{H}$ & 0.21557541194425 \\
\hline $\mathrm{C}$ & -3.80818208537913 \\
\hline $\mathrm{H}$ & -4.41280724279454 \\
\hline $\mathrm{H}$ & -4.17073024925531 \\
\hline $\mathrm{C}$ & -3.89506138780099 \\
\hline $\mathrm{H}$ & -4.93223201604278 \\
\hline $\mathrm{H}$ & -3.29032398195200 \\
\hline $\mathrm{H}$ & -3.53402845814990 \\
\hline $\mathrm{C}$ & 4.40673972174438 \\
\hline $\mathrm{H}$ & 5.08749513101148 \\
\hline $\mathrm{H}$ & 4.86182624634987 \\
\hline C & 4.14587302228689 \\
\hline $\mathrm{H}$ & 3.68222869708236 \\
\hline $\mathrm{H}$ & 5.08650000297303 \\
\hline $\mathrm{H}$ & 3.47771239434777 \\
\hline $\mathrm{C}$ & 1.26363751756890 \\
\hline $\mathrm{H}$ & 1.54192340144988 \\
\hline $\mathrm{H}$ & 2.16976242087668 \\
\hline $\mathrm{C}$ & 0.24323440281153 \\
\hline $\mathrm{H}$ & 0.68784857549036 \\
\hline $\mathrm{H}$ & -0.05609409022337 \\
\hline $\mathrm{H}$ & -0.65408121256281 \\
\hline $\mathrm{C}$ & 0.63606511334265 \\
\hline $\mathrm{H}$ & -0.23445224801875 \\
\hline $\mathrm{H}$ & 1.53318713991759 \\
\hline C & 0.70760057015123 \\
\hline $\mathrm{H}$ & -0.20157550807245 \\
\hline $\mathrm{H}$ & 4567802 \\
\hline & 1.55632 \\
\hline
\end{tabular}

$-3.37913782243379$

$-2.58736156026474$

$-3.98310331986167$

$-4.02026453964859$

$-2.88085114348294$

$-4.39079106325067$

$-4.92598737973916$

$-4.79081271618022$

$-4.61964823134946$

$-2.65557015238747$

$-3.21480996261358$

$-1.59956282934211$

$-2.99165551306200$

$-2.42152417880128$

$-1.37949674805165$

$-3.03367493359725$

$-2.51362604994156$

0.61957182454036

$-0.28743582797620$

1.14334176270788

1.51497496975954

1.81643793786043

2. 41230305117722

0.99892970932554

0.62494608739787

1.13535758910714

$-0.33101876232462$

1. 47603805086454

2. 42576026474059

1. 68680338437946

0.95912181526892

1. 74574620709237

0.89780833426342

2. 32582014798371

2. 62605090477722

3. 02198936601559

3. 47157505483689

2. 06434009789044

$-4.91415801663853$

$-5.20478198180452$

$-5.06042796872807$

$-5.73308582669783$

$-5.61757995763847$

$-6.79251525392766$

$-5.42275434778214$
3.75714069719335

3.59803334282065

4.61071333530515

2.87164835421180

4.81775455322457

4.55787640288795

5.51109571172881

3.97571930657689

4.02815428864133

5.60216947367947

6.54306098696086

5.83963916903915

5.01990112465874

5.68588665924118

5.99770085548105

6.59030533108922

5.13413133449125

$-0.16136043242986$

$-0.29375381275518$

0.72866756338606

$-1.38120466966032$

$-1.55201006356060$

$-1.23923040401061$

$-2.27339891057111$

$-1.30967550508118$

$-0.61565233313138$

$-1.58818848037910$

$-2.53563724591888$

$-2.26054167125262$

$-3.05232006274107$

$-3.22627967178738$

4.59467934088867

5. 22422361706878

4. 38869850287314

5.30385429356675

6.22298165762232

4. 68097252424781

5.56609597504204

$-0.09653386265690$

$-0.69937964468240$

$-0.70681102998206$

1.17688181187362

1.77114488707911

0.93128487875374

1. 78949964474315

\subsection{Compound 5Pr}

Final single point energy: -3199.741045957720

$$
\begin{array}{ll}
\text { C } & -2.03183350405360 \\
\text { C } & -2.03947025842493 \\
\text { C } & -2.12881990221603 \\
\text { C } & -2.22536105459121 \\
\text { C } & -2.10969715058403 \\
\text { C } & -2.03047007876969
\end{array}
$$

$$
\begin{array}{r}
-2.07741016604296 \\
-2.65625616893367 \\
-1.90204847525351 \\
-0.50835674163043 \\
0.10520692181224 \\
-0.67990298942074
\end{array}
$$

3.50521194121688
2.23500597946491
1.05981243257639
1.15975879281579
2.41934134704775
3.56639454418804 
$-0.52464877564718$

$-1.90753139419308$

0.25496607930325

$-2.51848402794003$

$-0.37401921921498$

$-1.77689967253288$

3.62800019465243

3. 30833697934229

3.80811758506493

2.39981267726566

3.27125077130516

1. 97126674808114

2. 34004038673638

3. 56711479671501

$-2.28918064828109$

$-1.60911458997781$

$-2.69418394277658$

$-1.29500081314435$

$-1.33352627948842$

$-2.27923658235185$

$-1.59913204273205$

$-1.30820603257299$

1. 13536232467909

$-3.49454981901225$

$-2.59587577400218$

$-3.67379221011125$

$-2.31884196283268$

1.89688649550584

1.76549351563584

2. 25270226287277

2. 04190467693304

$-2.65841327031954$

0.24018561149518

$-3.73201996283557$

$-0.17290906162428$

$-0.01235003916194$

$-3.59466955601471$

0.39394806440445

4.88316201910485

5. 30099377564361

6.08279401149947

5.70219097324933

4.46417684729969

4.34936535592961

3. 44962747592608

4. 09567869217175

5. 10284477201984

6.14013558071045

6.54498631167349

6.91939438976455

5.91562420279137

$-0.62986147263766$

0.74511835061721

0.65081743423324

1. 22794142501219

1.40406677695409

$-0.41560600037887$

0.02991532338792
2.87229616217462

2.80988492903502

1.74603895245007

1.56761977410034

0.49824710321050

0.42272478177233

0.45087616432405

0.76672345957083

0.13581440831103

1.81907440443827

0.53606222480254

2. 28488301229228

1. 57428706608386

0.02572907295178

$-1.18852143391372$

$-2.39739392068206$

$-0.83237065306978$

$-3.25373610949980$

$-2.66384681107611$

$-1.61036873635557$

$-2.80624251687305$

$-3.38749509410336$

3. 37342132957983

0.27526793833372

$-0.29633985125039$

$-0.12061811830483$

$-0.84429407320245$

2. 60920650414548

1.87788065091923

1.19920930892368

2. 88537798444595

$-1.13902111077722$

0.02033385993391

2.13105322479780

4.52008960455829

3.81972577949542

1.46517747310056

$-0.63572177609144$

$-0.95944776930755$

$-1.53223178059340$

$-2.28409854328305$

$-0.75699456631887$

$-2.01823516099045$

$-2.12359926545367$

$-2.55582663111703$

$-1.79470530996237$

$-2.91372363346560$

$-0.35697018399861$

0.46470759690376

$-1.11888913625491$

0.03327174664757

$-4.62861856059641$

$-4.65701103551489$

$-4.48566263936248$

$-5.63021182902386$

$-3.88479474957298$

$-4.95947022586962$

$-5.95423603040569$ 


\begin{tabular}{|c|c|}
\hline $\mathrm{H}$ & -2.31572593911864 \\
\hline $\mathrm{H}$ & -2.24388145741624 \\
\hline $\mathrm{C}$ & 0.33160350069672 \\
\hline $\mathrm{H}$ & -0.14957835869775 \\
\hline $\mathrm{H}$ & 0.19001845549033 \\
\hline $\mathrm{H}$ & 1.40335887398805 \\
\hline C & 4.25138848154287 \\
\hline C & 4.35746156965623 \\
\hline $\mathrm{H}$ & 5.10236667278665 \\
\hline $\mathrm{H}$ & 3.40018283120331 \\
\hline $\mathrm{H}$ & 4.66308490206770 \\
\hline C & 3.23450555857881 \\
\hline $\mathrm{H}$ & 2.23735890681445 \\
\hline $\mathrm{H}$ & 3.16278809270997 \\
\hline $\mathrm{H}$ & 3.53514020895066 \\
\hline C & 5.64078272050793 \\
\hline $\mathrm{H}$ & 6.38491054019192 \\
\hline $\mathrm{H}$ & 5.97672721855980 \\
\hline $\mathrm{H}$ & 5.61593360094973 \\
\hline C & -2.01355178787592 \\
\hline $\mathrm{C}$ & -1.93501592878984 \\
\hline $\mathrm{H}$ & -1.93457327362520 \\
\hline $\mathrm{H}$ & -2.79104934147785 \\
\hline $\mathrm{H}$ & -1.02022993727831 \\
\hline C & -3.31161100291760 \\
\hline $\mathrm{H}$ & -3.31721149978637 \\
\hline $\mathrm{H}$ & -3.41130743646888 \\
\hline $\mathrm{H}$ & -4.19300615205747 \\
\hline $\mathrm{C}$ & -0.79298497989362 \\
\hline $\mathrm{H}$ & -0.83464342848096 \\
\hline $\mathrm{H}$ & -0.75386740029268 \\
\hline $\mathrm{H}$ & 0.13705136083501 \\
\hline C & -3.79322418414841 \\
\hline $\mathrm{H}$ & -4.40215756007319 \\
\hline $\mathrm{H}$ & -4.16887877997629 \\
\hline C & -3.86771275202058 \\
\hline C & -5.30141417378777 \\
\hline $\mathrm{H}$ & -3.22401647289860 \\
\hline $\mathrm{H}$ & -3.45022914890414 \\
\hline $\mathrm{C}$ & 4.37241693495515 \\
\hline $\mathrm{H}$ & 5.05456190950943 \\
\hline $\mathrm{H}$ & 4.83926493563860 \\
\hline C & 4.09785515846324 \\
\hline $\mathrm{H}$ & 3.59366451405839 \\
\hline C & 5.37878492772456 \\
\hline $\mathrm{H}$ & 3.39483838336506 \\
\hline C & 1.29434500261045 \\
\hline $\mathrm{H}$ & 1.58473761166316 \\
\hline $\mathrm{H}$ & 2.20031225483570 \\
\hline C & 0.32862758813884 \\
\hline $\mathrm{H}$ & 0.72138696170177 \\
\hline $\mathrm{C}$ & 0.16231303663940 \\
\hline $\mathrm{H}$ & -0.64142420107494 \\
\hline $\mathrm{C}$ & 0.60922346869561 \\
\hline $\mathrm{H}$ & -0.30913061314121 \\
\hline $\mathrm{H}$ & 1.45222460846931 \\
\hline C & 0.79434828333773 \\
\hline $\mathrm{H}$ & 5.15865408086731 \\
\hline
\end{tabular}

$-1.35859636020625$

0.26195600816572

$-1.53688158923644$

$-2.51878023326948$

$-1.08523884710697$

$-1.69106113957956$

$-2.75117265459307$

$-1.91047804084094$

$-1.11526609080278$

$-1.45476586010388$

$-2.55074377497152$

$-3.88670335396759$

$-3.48349477188893$

$-4.56162700145866$

$-4.48381854883711$

$-3.36934991704453$

$-2.58928638464738$

$-3.96725591668718$

$-4.02132233219154$

$-2.89864138431732$

$-4.41376766082526$

$-4.95016538611096$

$-4.77235764151953$

$-4.68718043756097$

$-2.61523211754983$

$-3.17949653596746$

$-1.55508350542692$

$-2.90814822961711$

$-2.49666304052931$

$-1.44723553871474$

$-3.10198497315508$

$-2.64702237871701$

0.61708122266902

$-0.28701368706659$

1. 14449001954188

1.51355678389366

1. 96142229700197

2. 38175186775846

0.97798096982397

0.64723932009201

1. 17545311935781

$-0.30585563042462$

1. 48127024603014

2.40545042619111

1.80260964200800

0.93568554805390

1.64068535980596

0.73784571020026

2.22542174638235

2. 46265367974974

2. 42812264929732

3. 92908309316735

1.95919901400591

$-4.89479179253741$

$-5.20329992590378$

$-5.05753591076275$

$-5.69090974071322$

2. 40163373546480
$-4.96569072952520$

$-4.24977372590511$

$-5.72607180262811$

$-5.72833426684864$

$-6.71354645794558$

$-5.58035738040127$

4.02644645507125

5.31237966935965

5.21824604399172

5.58122633576894

6.14476258630202

4. 27615393059091

4. 46717986828824

3. 42041469329991

5.14354786805806

3.74010015156934

3. 55555937760873

4.59379743103270

2.86302619541103

4.80752789422077

4.54543900232855

5.49821286460525

3. 96626863792370

4. 01178065368420

5.60128828190575

6.53913037544582

5.84578301458080

5.02312173376598

5.66738699803308

5.96725566121762

6.57819601295219

5.11332047113014

$-0.19357476398293$

$-0.33465784214733$

0.69009582672311

$-1.41808817450072$

$-1.71947925761710$

$-1.25034379081420$

$-2.27643818837929$

$-1.31001846991095$

$-0.62927948066137$

$-1.58226211506777$

$-2.54908369735969$

$-2.25018815768786$

$-3.32556861333276$

$-3.18407564025959$

4. 60623309102487

5.14724683715015

4.41041477568544

5.46434186653171

6.48924364651029

5.05145945518699

5.48339977211499

$-0.05392811704959$

$-0.57340776433809$

$-0.73488036118829$

1. 22655200996978

$-4.21269404512246$ 


$\begin{array}{ll}\mathrm{H} & 5.88285537385543 \\ \mathrm{H} & 6.08768098200733 \\ \mathrm{H} & -5.33289929672973 \\ \mathrm{H} & -5.72738488646384 \\ \mathrm{H} & -5.95612014325921 \\ \mathrm{C} & 0.89508787149183 \\ \mathrm{H} & -0.04278916746784 \\ \mathrm{H} & 1.69673559778980 \\ \mathrm{H} & -0.00960046097077 \\ \mathrm{H} & 1.02983962381377 \\ \mathrm{H} & 1.74269290200231 \\ \mathrm{H} & -0.23847317931397 \\ \mathrm{H} & 1.12185520790612 \\ \mathrm{H} & -0.52202684473563\end{array}$

0.89017993429321

2.36796701640660

2.60344248650067

2. 52603312321398

1.10485524753929

$-7.19591453758687$

$-5.48060218086117$

$-5.33257012058777$

$-7.57814611706147$

$-7.75096015209652$

$-7.42904029448872$

4.02532871544325

4.45584527533885

4.44500536141108
$-3.65926720585437$

$-2.71191866163525$

$-2.60363416160096$

$-0.88389343745363$

$-1.90845532701628$

0.95905250760936

1.89880222136470

1.72995389252834

0.47497494825970

1.89044002679673

0.30673442758379

4. 04213535535124

5.08243602422508

5.73091044515051

\subsection{Compound 6Et}

Final single point energy: -3042.571217577515

$\begin{array}{ll}\mathrm{C} & -4.62865629539156 \\ \mathrm{C} & -4.10219338371045 \\ \mathrm{C} & -2.95269590988402 \\ \mathrm{C} & -2.30815020080542 \\ \mathrm{C} & -2.72935972250386 \\ \mathrm{C} & -3.88595906281884 \\ \mathrm{C} & 2.13169669238631 \\ \mathrm{C} & 1.29015124322823 \\ \mathrm{C} & 3.48121787458220 \\ \mathrm{C} & 1.83243769283708 \\ \mathrm{C} & 4.03920130613271 \\ \mathrm{H} & 4.11205471272499 \\ \mathrm{C} & 3.19113739597449 \\ \mathrm{H} & 3.56614395562329 \\ \mathrm{H} & 0.64806638196962 \\ \mathrm{C} & 0.85783835100805 \\ \mathrm{C} & 2.17680385130500 \\ \mathrm{C} & -0.22312995766455 \\ \mathrm{C} & 2.35588386825604 \\ \mathrm{C} & 0.00030524198766 \\ \mathrm{C} & 1.29536188754104 \\ \mathrm{H} & 3.34978968156496 \\ \mathrm{C} & -1.05958287188411 \\ \mathrm{C} & 0.03303405541051 \\ \mathrm{C} & -0.85958598028101 \\ \mathrm{C} & 1.32006068928466 \\ \mathrm{H} & -0.15254716730817 \\ \mathrm{C} & 0.44352263781806 \\ \mathrm{C} & 1.50143227472374 \\ \mathrm{H} & 2.48556628461699 \\ \mathrm{O} & -1.04183575768553 \\ \mathrm{O} & -0.04236645483400 \\ \mathrm{O} & -1.94797839011770 \\ \mathrm{C} & -2.43686281334915 \\ \mathrm{H} & -3.16886275701537 \\ \mathrm{H} & -2.39519435061478 \\ \mathrm{~S} & -1.87645634947035\end{array}$

0.49811521811486

0.19022944136524

0.79285563500838

1. 77102675571017

1.99325674120009

1.36745196484013

$-1.69997402402767$

$-2.21557114224203$

$-2.05119434245685$

$-3.00390536114196$

$-2.89028591889201$

$-1.65873335756574$

$-3.34026418712033$

$-3.96079424276885$

4. 17074552820256

3.19511299603244

2.73195225031434

2.43169768857311

1. 43283404326231

1.17046283586312

0.63960182725514

1.00435736192133

$-0.23308613062803$

0.45679552638359

$-1.52322037542464$

$-0.08326389318513$

1.44476437900884

$-2.04439416270117$

$-1.33650543212135$

$-1.78737097504278$

0.46163800101175

$-1.90783068513576$

$-2.24366752083098$

0.41814950423429

$-0.25360176818462$

1. 31092586378589

3.13764839488707
0.41494697554201

$-0.84634072167419$

$-1.35665790562719$

$-0.57960004925310$

0.73933156970602

1.21491956373861

2.02898522475964

1.02864412226861

2.01575451462062

0.00502220111439

1.03984263167908

2. 80696751580254

0.02554422158418

$-0.77687931217655$

1. 12667734012248

1.54645085365133

1.64737293208875

1. 97954586176958

2.12207223137585

2.56271564976656

2. 57649571757293

2.15952783557251

$-2.76439451542766$

$-3.29881798974080$

$-2.25404017899371$

$-3.34238448951823$

$-3.69708445295856$

$-2.18505546886208$

$-2.75094732460708$

$-2.71597482012023$

3.11964232212802

1.05602489995411

$-1.81482881514916$

$-2.74140558496017$

$-3.19855334851765$

$-3.37182486657413$

1. 84180571848608 
$-0.76810290055964$

$-0.71422513890397$

$-1.17763421326728$

$-3.60621901282506$

2.50259720725892

$-0.53531582138921$

1. 58737820283710

$-3.27102035207558$

$-4.02595127020475$

$-4.30697654829813$

$-3.41273798871994$

$-4.93760411734246$

$-4.18213487811133$

$-5.11999022554285$

$-3.69294437571277$

$-4.43274831029715$

$-1.99250128864435$

$-1.44490768285969$

$-1.31694409702370$

$-2.25148568354907$

0.62137538398831

0.76199250841509

1.33869444657646

$-0.20971889561683$

1.27579046221341

$-0.22619249477977$

$-0.33449839475446$

0.25037067797573

$-1.22869222266986$

2.02679106131812

2. 48299939219682

1. 99237516758424

2. 68292095276912

3. 65299432836286

4.83667137330686

5.51477886130656

5.41161122496774

4. 48048060108621

4.20339364922312

4.80043286618108

4.84698044423603

3. 38949881802029

2. 92478499732512

2. 56596807598560

2. 07207106187005

3. 61551930800771

$-0.09283209073313$

0.46033072506229

0.06815587149476

1. 55233353671797

0.17956404202542

0.29026238122387

$-0.07532829281297$

1. 37254656557105

$-0.15666172893889$

$-1.63384059165721$

$-2.04115218792795$

$-1.97935890037431$
3. 09186875533275

3. 91722799566461

3.49159656194898

$-1.34531149165299$

$-1.13852206772149$

$-1.47369929014952$

2. 22809530269868

1. 11237338320361

2.43600814741735

2. 50630348766681

3.30812236408695

2.49300924997500

$-0.05301856937243$

$-0.06368869248414$

$-1.02241469449070$

0.04652404393515

1.06833414095626

0.13470976915508

1. 89554843394710

1.13466360720272

$-4.01795828816304$

$-3.01605883268767$

$-2.14015482142845$

$-2.66643745084489$

$-3.48597244397244$

$-5.22557871933223$

$-5.96149271153562$

$-5.71881425529426$

$-4.91792521889195$

$-4.52893626351892$

$-5.00335012483528$

$-5.27518703131495$

$-3.71369219540881$

1. 26563525595301

2. 26133696030287

2.01057361928520

2. 24377421325188

3. 28450459789403

$-0.16561769856509$

$-0.25973557970768$

$-0.43480870664924$

$-0.89300828021977$

1. 32165858856249

2.32878239483941

0.63766822206315

1.03245352031870

0.85732354746779

$-0.07494791559072$

0.21794769627808

$-0.02583961025049$

$-1.11670082735390$

2.30502871154654

3.01839389949795

2. 42986091086318

2.57194715586612

0.76332321723830

1.06104832803917

$-0.25263128633107$ 


$\begin{array}{ll}\mathrm{H} & -5.20007761940529 \\ \mathrm{C} & -2.35171165016539 \\ \mathrm{H} & -2.62721185594875 \\ \mathrm{H} & -1.51274882493262 \\ \mathrm{C} & -3.52095160866387 \\ \mathrm{H} & -3.23641164277283 \\ \mathrm{H} & -4.36937725731271 \\ \mathrm{H} & -3.83991264743229 \\ \mathrm{C} & -1.71352177439601 \\ \mathrm{H} & -2.54374687491089 \\ \mathrm{H} & -2.07925153853473 \\ \mathrm{C} & -0.54149913631368 \\ \mathrm{H} & -0.19548748915353 \\ \mathrm{H} & -0.83975358332216 \\ \mathrm{H} & 0.29337421255807 \\ \mathrm{C} & -0.86602850773843 \\ \mathrm{C} & -1.29645677934874 \\ \mathrm{H} & -0.38347524113808 \\ \mathrm{H} & -1.55390270749477 \\ \mathrm{C} & -2.43139741102450 \\ \mathrm{H} & -2.63807990270670 \\ \mathrm{H} & -3.33992939231681 \\ \mathrm{H} & -2.17775698534778 \\ \mathrm{H} & -0.52835836445227 \\ \mathrm{C} & -2.30292840389378 \\ \mathrm{H} & -2.40052279134524 \\ \mathrm{H} & -2.62211746094128 \\ \mathrm{H} & -2.94682047635768 \\ \mathrm{H} & -0.73683953482106\end{array}$

$-2.05797753004953$

$-3.30362702671655$

$-2.87346533165645$

$-3.98896600798470$

$-4.03784946038320$

$-4.45843884366035$

$-3.36709355452761$

$-4.85556468284897$

3. 79991028959818

3. 67086192091294

4.38403146090276

4.49367502012170

3.93642682733294

5.49362454193823

4.59174794873958

$-2.91691151748645$

0.80806102957207

0.64255695706501

1.87109975752303

$-0.05592155604651$

0.18488361126256

0.11682345255050

$-1.11693966261472$

$-3.05871714855154$

$-2.44798352246634$

$-1.48151035419038$

$-2.34231353475142$

$-3.17693730248339$

$-3.86313762812170$
1.41900825803610 $-2.70219477619986$ $-3.67408775321331$ $-2.86460192515307$ $-2.07707441888708$ $-1.11035891404121$ $-1.92469700624958$ $-2.72971199741098$ $-1.60365470720232$ $-2.30984950058763$ $-0.75296478083973$ $-2.26914724131271$ $-3.14162468799513$ $-2.59636563767290$ $-1.57311569139076$

1.68233084675884 4.49546378274809 5.08252988656490 4.55813796404827 5.00840384215080 6.05506142977231 4. 42829078993813 4.94214763105914 2. 71784026077741 1. 63102120989989 2.12655394469132 0.59410032513224 2.13438517444408 1.14717180830026

\subsection{Compound 6Pr}

Final single point energy: -3199.748265364520

$\begin{array}{ll}\text { C } & -4.63165902976170 \\ \text { C } & -4.09333066366002 \\ \text { C } & -2.92729022555799 \\ \text { C } & -2.27723683369549 \\ \text { C } & -2.71140921988483 \\ \text { C } & -3.88520250986971 \\ \text { C } & 2.14030573386952 \\ \text { C } & 1.30035547065005 \\ \text { C } & 3.50412447052934 \\ \text { C } & 1.85425078434702 \\ \text { C } & 4.07744886912390 \\ \text { H } & 4.13511643970404 \\ \text { C } & 3.22740124492905 \\ \text { H } & 3.61146730767504 \\ \text { H } & 0.67955843812867 \\ \text { C } & 0.88123201543237 \\ \text { C } & 2.19741787931530 \\ \text { C } & -0.20669102674960 \\ \text { C } & 2.36670927537770 \\ \text { C } & 0.00516639827603 \\ \text { C } & 1.29900536736963 \\ \text { H } & 3.35887994041707\end{array}$

0.55983700277818

0.23396016411480

0.81386076872871

1. 78617858386297

2.02316183947622

1. 42102915003276 $-1.69027245572433$

$-2.24331227692536$

$-1.97854663928725$

$-2.99626214974936$

$-2.78489850691503$

$-1.56214136554222$

$-3.26716181989459$

$-3.86495473746333$

4.18113435419848

3. 20163863910083

2. 73231703984198

2.43908894943782

1. 43044242279970

1. 17064149171541

0.63705757184375

0.99949355097384
0.40016139780240 $-0.85137271063353$ $-1.34968896833420$ $-0.57063772401675$ 0.74151676198534 1.20536445761577 2.02640916629566

1.04527312494693

1.97832807086591

0.00183100041465

0.98417876398850

2.75687060248143 $-0.01354794493676$ $-0.82872862140078$ 1.16223909780528 1.57713273889707 1.68573642976270

1.99380306477081 2.15476579685279 2.56729267661858 2. 59223728082988 2. 20026556574133 


\begin{tabular}{|c|c|}
\hline C & -1.03885740895993 \\
\hline C & 0.05710882752706 \\
\hline $\mathrm{C}$ & -0.86057350664061 \\
\hline C & 1.33196080474531 \\
\hline $\mathrm{H}$ & -0.11818946793572 \\
\hline C & 0.43656266518311 \\
\hline C & 1.49522271152293 \\
\hline $\mathrm{H}$ & 2.46980017755193 \\
\hline O & -1.04557475051081 \\
\hline O & -0.04877608150803 \\
\hline O & -1.97467238069372 \\
\hline C & -2.40455961472854 \\
\hline $\mathrm{H}$ & -3.14212045761771 \\
\hline $\mathrm{H}$ & -2.34398613871598 \\
\hline S & -1.85340018315264 \\
\hline C & 1.56811955094672 \\
\hline $\mathrm{H}$ & 2.27810102430269 \\
\hline $\mathrm{H}$ & 0.63729402708348 \\
\hline S & 0.81656199540144 \\
\hline O & -1.23320515311866 \\
\hline $\mathrm{H}$ & -4.60157659855049 \\
\hline $\mathrm{H}$ & -4.20068016123707 \\
\hline C & 5.58509840411451 \\
\hline C & 5.91459274047664 \\
\hline $\mathrm{H}$ & 6.98268096727789 \\
\hline $\mathrm{H}$ & 5.66463251436546 \\
\hline $\mathrm{H}$ & 5.35622914710817 \\
\hline C & 6.02870117577700 \\
\hline $\mathrm{H}$ & 5.50972386051996 \\
\hline $\mathrm{H}$ & 5.85329891726406 \\
\hline $\mathrm{H}$ & 7.10060266713619 \\
\hline C & 6.39620917110202 \\
\hline $\mathrm{H}$ & 6.18760693295792 \\
\hline $\mathrm{H}$ & 6.16096889466996 \\
\hline $\mathrm{H}$ & 7.46942544310062 \\
\hline $\mathrm{C}$ & 2.52537515168758 \\
\hline C & 3.70238013199677 \\
\hline $\mathrm{H}$ & 3.40938021235965 \\
\hline $\mathrm{H}$ & 4.04432228449614 \\
\hline $\mathrm{H}$ & 4.55272170207182 \\
\hline C & 2.97464746826205 \\
\hline $\mathrm{H}$ & 2.16541460172005 \\
\hline $\mathrm{H}$ & 3.82881951521521 \\
\hline $\mathrm{H}$ & 3.27493529012056 \\
\hline $\mathrm{C}$ & 2.17130654003173 \\
\hline $\mathrm{H}$ & 3.04547576720066 \\
\hline $\mathrm{H}$ & 1.36495264327281 \\
\hline $\mathrm{H}$ & 1.86924964173684 \\
\hline $\mathrm{C}$ & 3.37859976462595 \\
\hline $\mathrm{C}$ & 3.41015831301193 \\
\hline $\mathrm{H}$ & 4.23643959264968 \\
\hline $\mathrm{H}$ & 2.48177012741480 \\
\hline $\mathrm{H}$ & 3.54627772809807 \\
\hline $\mathrm{C}$ & 3.21557636918286 \\
\hline $\mathrm{H}$ & 2.30687163171299 \\
\hline $\mathrm{H}$ & 4.06297555479906 \\
\hline $\mathrm{H}$ & 3.17072755825936 \\
\hline $\mathrm{C}$ & 4.73316897513826 \\
\hline
\end{tabular}

$-0.24739443281692$

0.42064912122927

$-1.54564502716104$

$-0.14468652046101$

1.41186146975375

$-2.08862885168322$

$-1.40227405319264$

$-1.87459501657724$

0.45828530061292

$-2.01845395658512$

$-2.21009348300540$

0.42641718297131

$-0.23672661853939$

1. 31752016408457

3.15826532220022

$-0.77601064810132$

$-0.73007857341543$

$-1.18921776731177$

$-3.63510715612519$

2.49759709222903

$-0.48719444309782$

1. 65229038484015

$-3.09898343469959$

$-3.86176514507319$

$-4.09689046049347$

$-3.27322107090425$

$-4.79995722855994$

$-3.97178929962123$

$-4.93383423499546$

$-3.47441528165722$

$-4.17606136934961$

$-1.78276916453623$

$-1.22306826784896$

$-1.13485995085413$

$-1.99656726405067$

0.53929576467143

0.67816774632350

1.27325696723953

$-0.29377830342390$

1. 17119672366336

$-0.32425761227212$

$-0.43234398196213$

0.13977536982692

$-1.32653803911441$

1. 94295517949139

2.38408394139192

1. 90878209776709

2. 61195302882249

3. 64684226335593

4.84767655490099

5.52116437289292

5.42250679557635

4.50911585476767

4.17156721321193

4.76638069395681

4.81071957402122

3. 34496333783696

2. 91907396450053
$-2.74598684595096$

$-3.29717509846423$

$-2.23860185096111$

$-3.37022364511633$

$-3.69249759506399$

$-2.19245650380031$

$-2.78652411708224$

$-2.77112847532179$

3.10244810993434

1.11542211998164

$-1.79262738344244$

$-2.72747109718766$

$-3.18740038533961$

$-3.35877885707204$

1. 84833711073666

3.09821996874980

3.93058097694426

3. 48350026500632

$-1.32910441847937$

$-1.12147893133284$

$-1.48118819394200$

2. 21302445852596

1.02081111555296

2. 32611973692949

2. 37081932597444

3. 21250713121374

2.37981476876193

$-0.16796140508601$

$-0.18180930073323$

$-1.12646690656665$

$-0.09454468078980$

0.98082086514979

0.06432796160577

1. 82890952677155

1.01283956930484

$-4.06295858249843$

$-3.07024735815139$

$-2.20157097692466$

$-2.70747407849314$

$-3.55285684296305$

$-5.26552720066943$

$-5.99391208258363$

$-5.77005490370461$

$-4.95107233515625$

$-4.58621609319928$

$-5.07361391966376$

$-5.32477462954785$

$-3.77594330249833$

1. 30960927730735

2. 28509458754182

2. 03460432892063

2.24374354296238

3. 31636706472258

$-0.13411537776978$

$-0.25136420284823$

$-0.40218340537898$

$-0.84795272087566$

1.40025896471275 


\begin{tabular}{|c|c|}
\hline $\mathrm{H}$ & 4.94198114471825 \\
\hline $\mathrm{H}$ & 4.77875180891349 \\
\hline $\mathrm{H}$ & 5.53632595965350 \\
\hline $\mathrm{C}$ & -6.00230167639545 \\
\hline $\mathrm{C}$ & -7.08533491537273 \\
\hline $\mathrm{H}$ & -8.07666401334026 \\
\hline $\mathrm{H}$ & -7.10233602646709 \\
\hline $\mathrm{H}$ & -6.90478217414114 \\
\hline C & -6.36709693313389 \\
\hline $\mathrm{H}$ & -5.63648585295845 \\
\hline $\mathrm{H}$ & -6.43879573063926 \\
\hline $\mathrm{H}$ & -7.34017942331797 \\
\hline C & -6.01612929457890 \\
\hline $\mathrm{H}$ & -6.99383886074872 \\
\hline $\mathrm{H}$ & -5.82158835783379 \\
\hline $\mathrm{H}$ & -5.25801653600268 \\
\hline C & -2.26281083939754 \\
\hline $\mathrm{H}$ & -1.72642051326424 \\
\hline $\mathrm{H}$ & -1.90689769239849 \\
\hline $\mathrm{C}$ & -3.76502577928545 \\
\hline $\mathrm{H}$ & -4.27200132687984 \\
\hline $\mathrm{H}$ & -4.09311202761131 \\
\hline C & -4.15306481140702 \\
\hline $\mathrm{C}$ & -1.63625904309638 \\
\hline $\mathrm{H}$ & -2.46498826521847 \\
\hline $\mathrm{H}$ & -1.99586807176451 \\
\hline $\mathrm{C}$ & -0.44847980584686 \\
\hline $\mathrm{H}$ & -0.12091123953042 \\
\hline C & -0.78125639908708 \\
\hline $\mathrm{H}$ & 0.38399937987616 \\
\hline C & -0.76720243333785 \\
\hline C & -1.32867842311773 \\
\hline $\mathrm{H}$ & -0.43128987152984 \\
\hline $\mathrm{H}$ & -1.58125510027160 \\
\hline C & -2.48418369008299 \\
\hline C & -2.84928528354213 \\
\hline $\mathrm{H}$ & -3.34622012951346 \\
\hline $\mathrm{H}$ & -2.21531732758197 \\
\hline $\mathrm{H}$ & -0.38409629577012 \\
\hline $\mathrm{C}$ & -2.24637205786486 \\
\hline $\mathrm{H}$ & -2.37762347281739 \\
\hline $\mathrm{H}$ & -2.58350003837417 \\
\hline C & -3.05077271774397 \\
\hline $\mathrm{H}$ & -0.58438441906000 \\
\hline $\mathrm{H}$ & -3.12712333671017 \\
\hline $\mathrm{H}$ & -2.01274494943055 \\
\hline $\mathrm{H}$ & -3.69574081228501 \\
\hline $\mathrm{H}$ & -2.92914339223197 \\
\hline $\mathrm{H}$ & -4.11891914774885 \\
\hline $\mathrm{H}$ & -2.73776886181353 \\
\hline $\mathrm{H}$ & -3.66140043052633 \\
\hline $\mathrm{H}$ & -5.23162323556534 \\
\hline $\mathrm{H}$ & -3.87101333151431 \\
\hline $\mathrm{H}$ & -1.61046509812613 \\
\hline $\mathrm{H}$ & 0.07915285388099 \\
\hline & -1.065579088559 \\
\hline
\end{tabular}

2.57309574961025

2. 05788230033277

3.60573310776980

$-0.00243719016013$

0.58506117246836

0.21203162879528

1. 67711121951370

0.31130359259035

0.37313994134064

$-0.01451714056399$

1. 45569967143060

$-0.05619136775693$

$-1.54182188884621$

$-1.93489910793784$

$-1.88026828847334$

$-1.98517209989987$

$-3.50122909285588$

$-3.60502720648902$

$-4.28291564887514$

$-3.62810557087528$

$-3.43332421808661$

$-2.85295994499406$

$-5.01402569705008$

3.79716223360374

3. 68011562430059

4. 39892392871664

4.47006840635604

3.86286265278085

5.89046612957610

4.48903764233118

$-3.08228607438884$

0.80063513772953

0.62588884170475

1.86552809779020

$-0.05548419055803$

0.24906432491297

0.11615462495647

$-1.11164381045521$

$-3.19622946650615$

$-2.74874020021858$

$-1.77703937195126$

$-2.63489961271405$

$-3.83309470472692$

$-4.02134235887708$

1. 29957510899031

0.04092479707449

$-0.36016543721375$

$-4.81158480762746$

$-3.60157658145126$

$-3.93508626687535$

$-5.23216905445740$

$-5.08369500917979$

$-5.80127349213049$

5.89194465597823

6.35180507685478

6.53145083263323
2.41620256601401

0.72777911033138

1. 11719920908224

0.82451309009025

$-0.11213269085975$

0.16666513959770

$-0.05168603695450$

$-1.15490977057749$

2.27316185655646

2. 98850800039126

2. 40832773450457

2.52781886225996

0.71311987000391

1.01043631700740

$-0.30692727135901$

1.36219212323487

$-2.36697574517387$

$-3.31728697274420$

$-1.68962644289276$

$-2.58358553225583$

$-1.63349380652003$

$-3.28409609287015$

$-3.11006064580648$

$-1.60035414985261$

$-2.31181464923402$

$-0.75796660005814$

$-2.26779023086042$

$-3.11672005316922$

$-2.73584972332143$

$-1.55913698019939$

1.77164361272775

4. 47231107603775

5.08248178851868

4. 53647296694775

4.96298421049942

6.41931533887550

4. 31158615720658

4.85611681214488

2. 79581791319794

1. 78521250624152

2.26776154309452

0.75200485118579

2.51008097898065

1.23657345865352

6.54980938655954

7.09421826885452

6.74618931583002

2.03300305927378

2. 51118875267689

3.55453976384135

$-4.06331460817384$

$-3.27161229248822$

$-2.40421519967862$

$-3.45101011546217$

$-3.22770043192192$

$-1.89567669899329$ 


\subsection{Compound 7Et}

Final single point energy: -3042.557968528724

\begin{tabular}{|c|c|}
\hline $\mathrm{C}$ & -4.73821112650029 \\
\hline$C$ & -4.22976546408514 \\
\hline $\mathrm{C}$ & -3.40970797064820 \\
\hline $\mathrm{C}$ & -3.09177688114578 \\
\hline C & -3.51975313230570 \\
\hline $\mathrm{C}$ & -4.34721676442054 \\
\hline 0 & 2.40718644606896 \\
\hline $\mathrm{C}$ & 2.54854412639603 \\
\hline C & 2.31377972917337 \\
\hline $\mathrm{C}$ & 2.41381451965967 \\
\hline$C$ & 2.34200276485990 \\
\hline $\mathrm{H}$ & 2.21849612765382 \\
\hline $\mathrm{C}$ & 2.35886289747371 \\
\hline $\mathrm{H}$ & 2.30570374265249 \\
\hline $\mathrm{C}$ & -1.39178758560394 \\
\hline $\mathrm{C}$ & -0.21621664681171 \\
\hline $\mathrm{C}$ & -1.31477843011366 \\
\hline $\mathrm{C}$ & 1.02624666359103 \\
\hline $\mathrm{C}$ & -0.09257732112247 \\
\hline$C$ & 1.05877640856294 \\
\hline $\mathrm{C}$ & -1.68135115069299 \\
\hline $\mathrm{C}$ & -1.64986283102344 \\
\hline $\mathrm{C}$ & -0.53751455191915 \\
\hline $\mathrm{C}$ & -0.52210321420173 \\
\hline $\mathrm{H}$ & -2.55517333056201 \\
\hline $\mathrm{C}$ & 0.64726913273629 \\
\hline $\mathrm{C}$ & 0.63642807875462 \\
\hline H & 1.56489645120740 \\
\hline O & 2.74818168679939 \\
\hline O & -0.59825466829737 \\
\hline $\mathrm{C}$ & -2.93608920880844 \\
\hline $\mathrm{H}$ & -3.75799986892848 \\
\hline $\mathrm{H}$ & -2.76478524593620 \\
\hline S & -3.02865421232451 \\
\hline $\mathrm{C}$ & 2.30856012013517 \\
\hline $\mathrm{H}$ & 2.37018334528484 \\
\hline $\mathrm{H}$ & 3.15765922361183 \\
\hline S & 2.21789303961610 \\
\hline O & -2.33503127435662 \\
\hline $\mathrm{H}$ & -4.49215392600343 \\
\hline $\mathrm{H}$ & -4.67980980307335 \\
\hline $\mathrm{H}$ & 2.02578792002002 \\
\hline $\mathrm{H}$ & -2.24379100309811 \\
\hline O & -0.24174541140709 \\
\hline $\mathrm{C}$ & -3.04773313803256 \\
\hline $\mathrm{H}$ & -3.96269843885559 \\
\hline $\mathrm{H}$ & -3.33262057990110 \\
\hline $\mathrm{C}$ & -2.12941756685877 \\
\hline $\mathrm{H}$ & -2.60058486592199 \\
\hline $\mathrm{H}$ & -1.19170187783724 \\
\hline $\mathrm{H}$ & -1.90432878725158 \\
\hline C & -0.40166104545484 \\
\hline $\mathrm{H}$ & 0.39918927882814 \\
\hline $\mathrm{H}$ & -1.35879419081268 \\
\hline
\end{tabular}

$-1.72051734478321$

$-2.22361470031502$

$-1.47922763065590$

$-0.15532448518908$

0.34733620413660

$-0.43070792832252$

0.84824839140525

$-0.53862958667256$

1. 37125090890376

$-1.37496098793778$

0.56797976832668

2.44441390035296

$-0.81511808587426$

$-1.49006217600193$

1.62589654595226

1. 98676132505471

0.95443941707501

1.54755101613674

0.58672264017468

0.86831074034222

$-2.92892167068492$

$-3.89809585369452$

$-2.74521171482906$

$-4.66858734708317$

$-4.04015182125252$

$-3.43727167313916$

$-4.38762663447364$

$-4.89974340843044$

$-1.02408437142052$

$-1.84658650728926$

$-2.05263154127232$

$-2.63523763658635$

$-1.21556309296486$

1.99087794423581

1.75975705889530

2.79517166707706

1. 58519989853552

$-3.16231170751177$

0.58516360603773

$-3.22910002386162$

0.00606595578284

0.54566532023751

0.72818337267610

2.75856594068900

1.61097027846016

1.18191247800260

2. 40744161156224

2.13684499820083

2. 97754403206309

2.47383732317698

1.36100786065453

4. 16521519567094

4. 49891396135252

4. 33472724819501
1.68880848235070

0.48221075860863

$-0.36247296064326$

$-0.00543535047779$

1.23175709554811

2. 05182562912716

0.30107314928870

0.45801491214749

$-0.99028867338532$

$-0.66212912728926$

$-2.13471514370832$

$-1.08450956689092$

$-1.93766716840516$

$-2.78285960413961$

2.49139939517120

1. 81858192077075

3.71750408153475

2.31377880377142

4.27697736840955

3.52953255167974

$-1.76250301168592$

$-2.77414170609509$

$-0.96717919729250$

$-3.06089147687455$

$-3.35153865596601$

$-1.30804486057058$

$-2.32783735936015$

$-2.55088341229957$

1. 72878643617111

0.05236962019753

$-1.68485159598576$

$-2.11284159410182$

$-2.36677280020534$

1. 81157720865308

1. 51712885251287

1.17408465758752

2.18223223663250

$-0.46774609911356$

$-0.87385134525357$

0.16815558888152

2.98292755792048

3. 89437789964888

4.22166453187002

0.67856879323240

$-1.59228470819327$

$-2.02148039144162$

$-0.89983105839287$

$-2.67717049436241$

$-3.19554028051311$

$-2.23381523689323$

$-3.41256913370928$

0.94415972256257

1.61753747046125

1.45059994647939 
4.92030549911436

5.99346424991840

4.74969633699710

4.60673377768255

$-2.28863805843233$

$-1.50234174362889$

$-2.28057743982530$

$-3.64567840365979$

$-3.83436048678261$

$-3.67466104521002$

$-4.45562338486533$

$-1.88270967652699$

$-2.89332215421138$

$-1.91512100879567$

$-1.36073681153038$

$-2.03021724701974$

$-0.36304646500304$

$-1.31239816413653$

$-5.76614164890982$

$-7.12557008318342$

$-7.92359388800688$

$-7.11231383340458$

$-7.37906855727056$

$-5.89972706047401$

$-6.16551428252374$

$-4.97636943052558$

$-6.69037855269978$

$-5.44404322630320$

$-6.22494965163198$

$-4.49067613509079$

$-5.37940411031379$

1.14338503466132

2.66847604310037

3. 19768664041309

3. 02956164554679

2.94718429183801

0.83629542389204

$-0.23983911695082$

1. 23062721736230

1. 29181048093445

0.49646364242995

0.66733773593552

0.92644894186114

$-0.58227515651148$

$-2.57185276859597$

$-2.85658483370422$

$-3.40160881591964$

$-3.46045301779626$

$-1.92431343403707$

$-1.85948345707953$

$-1.65045667603421$

$-0.91640969097885$

$-2.49786711627920$

$-3.91318005821977$

$-4.49909103339765$

$-3.74472453570721$

$-4.51893022066858$

$-0.11207048728935$
$-0.36943293280798$

$-0.18354278218885$

$-0.88600115810179$

$-1.02774824295469$

1.42136718927605

2.00727352856132

1.64463157490584

1.74862993341722

2.81977237360855

1. 51997207862476

1. 21023882754611

1. 95241693333953

2. 14925852117331

1.04782989059029

3. 12989916589831

3. 32848358015419

2. 92483682973764

4.03300742131836

$-4.14051616229532$

$-3.47702411373505$

$-4.22703260643035$

$-2.98394088490598$

$-2.72373967842410$

$-4.86175450366667$

$-4.16856864003472$

$-5.37563168538398$

$-5.61518943692540$

$-5.19981689358473$

$-5.96724134176228$

$-5.69389615536058$

$-4.75809762021666$

$-3.56308134173656$

$-3.58083036789574$

$-3.04253623501736$

$-4.61308916671855$

$-3.14025791111733$

$-4.18631998512535$

$-4.22967360934052$

$-5.20618920680831$

$-3.59906557355657$

$-4.43223777913323$

$-3.99775987631138$

$-5.43855759319513$

$-4.53300129838712$

2.53977173964955

1.72047745283596

0.79985056498873

2.30560652298575

1.44475765257048

3. 84194747371500

4.48206313552541

3. 64413531738793

4.40786083309774

2. 91901769105166

2.03682378714715

3. 49945874380793

3.52483469189136

5.64466286877194 


$\begin{array}{ll}\mathrm{C} & -1.33884712896461 \\ \mathrm{H} & -1.83301006122385 \\ \mathrm{H} & -2.01232469356263 \\ \mathrm{H} & -1.19880743680053 \\ \mathrm{C} & 0.65256908147608 \\ \mathrm{H} & 0.01544547043412 \\ \mathrm{H} & 1.63421090915351 \\ \mathrm{H} & 0.77259984086001 \\ \mathrm{C} & 0.93043148238339 \\ \mathrm{H} & 0.50542175876226 \\ \mathrm{H} & 1.03319272289460 \\ \mathrm{H} & 1.93258260217950\end{array}$

$-0.28645448189878$

0.67354909704863

$-0.91981243909828$

$-0.76597830808934$

$-1.51370215790151$

$-2.14231707515879$

$-1.45817986809394$

$-2.00917360457320$

0.73239175902257

1. 72745450736819

0.24717422109815

0.85956680860456
6.33559260825152

6.50729064470421

5.75139424133647

7.30876316766192

5.45842416982266

4.83020066995360

4.98238988255059

6.42688071428911

6.57355695692680

6.73185508646788

7.54933673515103

6.15721230445899

\subsection{Compound 7Pr}

Final single point energy: -3199.737217038280

$\begin{array}{ll}\mathrm{C} & -5.19665166786900 \\ \mathrm{C} & -4.66669679482817 \\ \mathrm{C} & -3.69939372687324 \\ \mathrm{C} & -3.22045545899398 \\ \mathrm{C} & -3.68727355446158 \\ \mathrm{C} & -4.67667259932741 \\ \mathrm{C} & 2.49477601461885 \\ \mathrm{C} & 2.46343349709876 \\ \mathrm{C} & 2.67524824705370 \\ \mathrm{C} & 2.44183007800940 \\ \mathrm{C} & 2.81164828227091 \\ \mathrm{H} & 2.71474410940472 \\ \mathrm{C} & 2.65309199215827 \\ \mathrm{H} & 2.68379290821598 \\ \mathrm{C} & -1.46755914484335 \\ \mathrm{C} & -0.24014740107630 \\ \mathrm{C} & -1.49693502358581 \\ \mathrm{C} & 0.95517328670734 \\ \mathrm{C} & -0.32830060501446 \\ \mathrm{C} & 0.88504469857144 \\ \mathrm{C} & -1.96321013471093 \\ \mathrm{C} & -1.95762825729532 \\ \mathrm{C} & -0.74282534145485 \\ \mathrm{C} & -0.78325647058043 \\ \mathrm{H} & -2.91849132315004 \\ \mathrm{C} & 0.46209676078309 \\ \mathrm{C} & 0.41044452284211 \\ \mathrm{H} & 1.35801370484828 \\ \mathrm{O} & 2.39713685300483 \\ \mathrm{O} & -0.90379517362355 \\ \mathrm{C} & -3.29854049120296 \\ \mathrm{H} & -3.37803220346569 \\ \mathrm{H} & -4.07751792558691 \\ \mathrm{~S} & -3.02324383021606 \\ \mathrm{C} & 2.30016589513988 \\ \mathrm{H} & 2.41162639745861 \\ \mathrm{H} & 3.08646220396953\end{array}$

$-1.48578818319284$

$-2.42831073653035$

$-2.12698499010484$

$-0.81025835780346$

0.14312189614101

$-0.19154326444081$

0.76314665270015

$-0.63231436499257$

1. 30494115117442

$-1.44103011457197$

0.51795163197231

2.38350691432751

$-0.86269360887374$

$-1.51824390410802$

1.53452375838333

1.85286605240107

0.97519971689572

1.50464207772183

0.68436023605927

0.93980179514136

$-3.28730250201776$

$-3.49529779681846$

$-3.25091921835774$

$-3.68671174493180$

$-3.54183801633998$

$-3.35461501109389$

$-3.60176545744916$

$-3.73096213229596$

$-1.17255522299523$

$-3.06727709788684$

$-3.25567822504688$

$-4.17995949809177$

$-3.33067973765168$

1.82079924378354

1.68177355126896

2.71324468066743

1. 50886038293561
2. 29157195278597

1. 40466511871131

0.44198259334848

0.37175371566355

1. 29404210301075

2.22205201539457

0.35500969746274

0.49490110995477

$-0.92046123770369$

$-0.65557403903910$

$-2.06603089394874$

$-0.99951210900448$

$-1.90679434948403$

$-2.76741093189401$

2.18181647200320

1. 58877852170798

3. 46418235622338

2. 24222821752369

4.16435760982748

3. 51225232541285

$-1.22834847013525$

$-2.60555064353266$

$-0.51794969342599$

$-3.34130618686935$

$-3.10379162340142$

$-1.24846113519444$

$-2.62795339974605$

$-3.13611276116717$

1.75931767346081

0.81821234502376

$-0.50545142838252$

0.07850051972830

$-1.27347095695743$

1. 30751190058977

1.55353831417316

1. 20805044462325

2. 29245334239197 
2.12696409241331

$-2.27687954868972$

$-5.02560957853727$

$-5.01815703894924$

1.81854438381506

$-2.46373183787209$

$-0.17253016617691$

$-2.80504195491072$

$-3.44720537333598$

$-3.41907336756774$

$-1.64231374344874$

$-2.12569229558737$

$-1.00956394768669$

$-1.03631298872772$

$-0.42321074348083$

0.34423066515221

$-1.39734271942014$

$-0.40421783761834$

$-0.65897399903589$

0.56324055461372

$-1.16380603404691$

$-0.04459104849437$

$-0.55044915229034$

0.92501631770857

0.09253729952994

0.78211096337059

$-1.22591903632170$

0.58628506112999

3.55554374929215

3.18994827215767

3. 92050932247893

4.67888104356304

5.89331204730619

4. 97217983674723

4.30326909651561

$-0.77077517254722$

$-0.14154906220472$

$-0.12166106433335$

0.88477637774778

$-0.71589432113596$

$-2.18743688450057$

$-2.82084540658815$

$-2.68300058407223$

$-2.12801642825401$

0.06308922796665

0.07069697412362

$-0.35257287427055$

1. 10078367155067

3. 13598857159166

3. 18253391567455

3. 95251043605461

3. 41671474526562

2. 22250221975465

4. 52203650252430

4.54011475659416

4.77549426899977

5.30181400990137

2. 07004649241060
$-3.22314773867980$

$-0.47044496512003$

$-3.45226232171132$

0.58379857593084

0.68186485926987

0.76359640772780

2. 47288228221272

0.11956172426082

$-0.61067333748229$

0.98915135625537

0.53205183775084

1. 18459112335633

1. 21803576882865

$-0.35259750024439$

3.88784799543394

4.37847075973368

4. 08186575711118

4. 42591378531109

5.93554449195310

4.19225827762987

3.89502488460525

$-3.61525119808392$

$-3.31224270489760$

$-3.12515120913002$

$-5.13370079834690$

$-5.41052890183202$

$-5.89610585894989$

$-5.42990416634530$

$-1.90752684766770$

$-2.55475839670662$

$-2.55182101085809$

$-1.00864255382856$

$-1.82467134139685$

$-0.31442244939672$

$-0.40283560586463$

$-4.00472267663840$

$-5.40083868446088$

$-5.64982874967429$

$-5.44176321278054$

$-6.17209999990379$

$-4.01777755608503$

$-4.77663612633400$

$-3.04741776253645$

$-4.24859912449130$

$-2.94588243234181$

$-3.16244663908397$

$-1.94484991535078$

$-2.92941879461858$

1.10504891390869

2. 64375831956176

3. 02325603198927

3. 01181756048655

3. 07626868199143

0.58599018925969

$-0.50485665904814$

0.98803354470771

0.89190628653081

0.66764778179716
$-0.54350002674335$

$-0.56045628554626$

1.44672310548174

2.89352899949020

3.99803074540187

3.89777744251456

0.36040005699166

$-1.76006705977563$

$-2.27315504259298$

$-1.49744002414173$

$-2.64444256158322$

$-3.94391170844550$

$-2.07467975415089$

$-2.86163534928779$

0.39562325601602

1.01098505627774

0.86157879963482

$-1.02507336427785$

$-1.07322464731724$

$-1.48116826819978$

$-1.60643808762990$

1.83923636828473

2.75976815524119

1.80600675990525

1.79006375982909

2. 59719404942845

1.95013255961707

0.85896049505972

2. 20122198627093

3.00261206748432

1.39485095563496

2. 71064567356215

3.16669917129700

1. 91655510371238

3. 54202000879738

$-4.84848082716022$

$-5.06808524772614$

$-6.13412880567917$

$-4.69589937478581$

$-4.54738945959448$

$-5.45276830431615$

$-4.98509111673925$

$-5.35268755356614$

$-6.52024656819526$

$-5.60555479486793$

$-6.67881171789575$

$-5.46244573650183$

$-5.26336880690161$

$-3.45280033956714$

$-3.43889081158368$

$-2.76154341861750$

$-4.44179098673531$

$-3.14423247213606$

$-3.90573310319808$

$-3.97156704907064$

$-4.89214115697805$

$-3.20243450164665$

$-4.48188698060561$ 
1.00551092356705

1.09587399306529

$-0.41807020359918$

$-1.89890877570780$

$-2.44765540616567$

$-3.32163191637865$

$-2.74381939067739$

$-1.68747033113071$

$-0.71742362534636$

$-0.30666444974748$

0.09129315435177

$-1.05302227663341$

$-2.99817138786161$

$-3.88549744300074$

$-2.63197673670138$

$-3.30748452480756$

0.10302328673036

$-0.15200127671715$

0.76967351451460

$-0.86717512296586$

$-0.56729019665386$

$-1.24203348562876$

$-1.96485866618474$

$-1.12506704117225$

$-1.66203618353967$

1.09832053625806

2.05600080395448

0.69936796314697

1.29197648115973

$-5.67195844604946$

$-5.63054407530686$

$-6.97626984861226$

$-2.42266205077529$

$-2.51044314891559$

$-1.17197889378834$

6.30198964647330

6.48945689014830

6.18832789470645

1. 47901694829601

2.08382645422028

0.50093589978369
$-4.18001938291627$

$-5.46442041671642$

$-4.59532683307274$

3. 29742388713391

2. 53069109761588

1.92646569759105

3.23318559388927

1.86079976883233

4.16601475223217

4.76968314252175

3. 55988618273151

4. 85317871368551

4. 23582763900348

3. 68198508151662

4. 79938092574061

4.95212754949430

5.59051161723456

6.11639398052820

6.16780515955113

5.49068977904510

7.12722127378997

5.61028407648222

4.93982368445981

5.29406739480923

6.62152430112193

6.54856158598689

6.56458748539549

7.56800278290808

6.24947946742374

1.13367420608387

2.88817415466249

1.95458423091771

2. 34583210834304

3. 97885360859880

3. 52864838004205

$-2.10257760965384$

$-0.51206370706293$

$-0.64854238084262$

$-4.57547985521922$

$-3.74362964257328$

$-4.52831123262346$ 